\title{
Geometric optimization for the maximum heat transfer density rate from cylinders rotating in natural convection
}

\author{
by \\ Logan Garrick Page \\ Submitted in partial fulfillment of the requirements for the degree
}

Masters in Engineering

in the

Faculty of Engineering, Built Environment and Information Technology

University of Pretoria

2012

UNIVERSITEIT VAN PRETORIA

UNIVERSITY OF PRETORIA

YUNIBESITHI YA PRETORIA 


\section{Abstract}

Title: Geometric optimization for the maximum heat transfer density rate from cylinders rotating in natural convection

Author: $\quad$ LG Page

Supervisors: Prof T Bello-Ochende and Prof JP Meyer

Department: Mechanical and Aeronautical Engineering

University: University of Pretoria

Degree: $\quad$ Masters in Engineering (Mechanical Engineering)

In this study we investigates the thermal behaviour of an assembly of consecutive cylinders in a counter-rotating configuration cooled by natural convection with the objective of maximizing the heat transfer density rate (heat transfer rate per unit volume). A numerical model was used to solve the governing equations that describe the temperature and flow fields and an optimisation algorithm was used to find the optimal structure for flow configurations with two or more degrees of freedom. The geometric structure of the consecutive cylinders was optimized for each flow regime (Rayleigh number) and cylinder rotation speed for one and two degrees of freedom. Smaller cylinders were placed at the entrance to the assembly, in the wedge-shaped flow regions occupied by fluid that had not yet been used for heat transfer, to create additional length scales to the flow configuration.

It was found that the optimized spacing decreases and the heat transfer density rate increases as the Rayleigh number increases, for the optimized structure. It was also found that the optimized spacing decreases and the maximum heat transfer density rate increases, as the cylinder rotation speed was increased for the single scale configuration at each Rayleigh number. Results further showed that there was an increase in the heat transfer density rate of the rotating cylinders over stationary cylinders for a single scale configuration.

For a multi scale configuration it was found that there was almost no effect of cylinder rotation on the maximum heat transfer density rate, when compared to stationary cylinders, at each Rayleigh number; with the exception of high cylinder rotation speeds, which serve to suppress the heat transfer density rate. It was, however, found that the optimized spacing decreases as the cylinder rotation speed was increased at each 
Rayleigh number. Results further showed that the maximum heat transfer density rate for a multi scale configuration (with stationary cylinders) was higher than a single scale configuration (with rotating cylinders) with an exception at very low Rayleigh numbers.

Keywords: Natural convection; Rotating cylinders; Heat transfer density rate; Counter-rotation; Optimal packing; Multi scale; Mathematical Optimization 


\section{Acknowledgements}

I wish to express my gratitude to my supervisor, Prof. T. Bello-Ochende, for his guidance, support, encouragement and friendship. It has been a real privilege working with him and the knowledge I have gained from the experience is truly invaluable.

I would also like to thank my co-supervisor, Prof JP Meyer, for his technical support, which has enabled the successful completion of this work, and for his assistance in arranging financial aid.

I would like to thank Dr N Wilke for his insight, useful comments and encouragement during the course of this work.

I would also like to thank $\mathrm{W}$ Leibbrandt for his guidance and instruction in teaching me how to program efficiently in python.

Lastly I would like to thank my parents, family members and friends for their encouragement and support during this period. 


\section{Table of contents}

Abstract $\quad$ i

Acknowledgements

Table of contents $\quad$ iv

List of figures viii

List of tables $\quad$ xi

Nomenclature $\quad$ xii

Chapter 1: Introduction $\quad 1$

1.1 Background .......................... 1

1.2 Research objectives . . . . . . . . . . . . . . . 6

1.3 Scope of study . . . . . . . . . . . . . . . . . 6

1.4 Structure of thesis . . . . . . . . . . . . . . 7

$\begin{array}{lll}\text { Chapter 2: } & \text { Literature study } & 9\end{array}$

2.1 Introduction . . . . . . . . . . . . . . . . . . 9

2.2 Constructal flow geometry . . . . . . . . . . . . . . . . . 9

2.3 Heat transfer devices . . . . . . . . . . . . . . . . . . . . . . . . 10

2.3.1 Increased geometric complexity . . . . . . . . . . . . . . 12

2.4 The effect of rotation . . . . . . . . . . . . . . . . . . 14

2.5 Conclusion . . . . . . . . . . . . . . . . . . . 15

$\begin{array}{ll}\text { Chapter 3: } & \text { Numerical modelling }\end{array}$

3.1 Introduction . . . . . . . . . . . . . . . . . 16

3.2 Overview of numerical modelling . . . . . . . . . . . . . . 16

3.3 Geometry and mesh generation . . . . . . . . . . . . 17

3.4 Governing equations . . . . . . . . . . . . . . . 18 
3.4.1 Conservation of mass . . . . . . . . . . . . . . . . . 19

3.4.2 Conservation of momentum . . . . . . . . . . . . . . . . 19

3.4.3 Conservation of energy . . . . . . . . . . . . . 20

3.5 Numerical solution schemes . . . . . . . . . . . . . . . . . 21

3.5.1 Finite volume method . . . . . . . . . . . . . . . 21

3.5.2 The PISO algorithm . . . . . . . . . . . . . . 22

3.6 Verification . . . . . . . . . . . . . . . . . . . 25

3.6.1 Self driven cavity . . . . . . . . . . . . . . . . 25

3.6.2 Parallel plates . . . . . . . . . . . . . . . . 26

3.7 Conclusion . . . . . . . . . . . . . . . . . . . . . . 28

$\begin{array}{lll}\text { Chapter 4: } & \text { Parametrisation and optimization }\end{array}$

4.1 Introduction . . . . . . . . . . . . . . . . . . . . . . 29

4.2 Open source tools . . . . . . . . . . . . . . . . . . . . . 30

4.3 Parametrisation . . . . . . . . . . . . . . . . . . . 31

4.4 Numerical optimisation . . . . . . . . . . . . . . . . . . . . 31

4.4.1 Numerical optimisation overview . . . . . . . . . . . . 33

4.4.2 Non-linear constrained optimisation . . . . . . . . . . . . 33

4.4.3 Numerical optimisation algorithm . . . . . . . . . . . . 35

4.4 .4 Automation . . . . . . . . . . . . . . 36

4.5 Conclusion . . . . . . . . . . . . . . . . . . 36

Chapter 5: Optimization of rotating cylinders in natural convection 38

5.1 Introduction . . . . . . . . . . . . . . . . . . . . 38

5.2 The numerical CFD model . . . . . . . . . . . . . . . . . . 38

5.2 .1 Single scale . . . . . . . . . . . . . . . . . . 39

5.2 .2 Multi scale....................... . . 41

5.3 Non-dimensional governing equations . . . . . . . . . . . . . . 43

5.4 Objective function . . . . . . . . . . . . . . . . . . . . 44

5.5 Convergence criteria and mesh independence . . . . . . . . . . . 46

5.5.1 Single scale . . . . . . . . . . . . . . . . . 47

5.5 .2 Multi scale. . . . . . . . . . . . . . . . . . 49

5.6 Conclusion . . . . . . . . . . . . . . . . . . 50

Chapter 6: Optimal geometric configuration results $\quad 51$

6.1 Introduction . . . . . . . . . . . . . . . . . . . . 51 
6.2 Scale analysis . . . . . . . . . . . . . . . . . . . 51

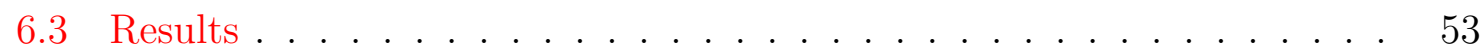

6.3 .1 Single scale . . . . . . . . . . . . . . . . . 54

6.3.1.1 Stationary cylinders . . . . . . . . . . . 54

6.3.1.2 Rotating cylinders .............. 55

6.3 .2 Multi scale . . . . . . . . . . . . . . . . . 60

6.3.2.1 Stationary cylinders . . . . . . . . . . . 60

6.3.2.2 Rotating cylinders . . . . . . . . . . . . 63

6.3.3 Observations . . . . . . . . . . . . . . . . 69

6.4 Conclusion . . . . . . . . . . . . . . . . . . . . . . . 69

$\begin{array}{lll}\text { Chapter 7: } & \text { Summary, conclusions and recommendations } & 71\end{array}$

7.1 Summary . . . . . . . . . . . . . . . . . 71

7.2 Conclusions . . . . . . . . . . . . . . . . . . . . . 71

7.3 Recommendations for future work . . . . . . . . . . . . . . . . . 72

7.3.1 Cylinder spacings and configurations . . . . . . . . . . . . 72

7.3.2 Optimisation algorithm . . . . . . . . . . . . . . . 74

7.3.3 Enhanced natural convection systems . . . . . . . . . . . . . . 74

$\begin{array}{ll}\text { Publications in Journals and Conferences } & \mathbf{7 5}\end{array}$

$\begin{array}{ll}\text { References } & 76\end{array}$

Appendix A: Nomenclature $\quad$ Ai

Appendix A: Discretisation methods $\quad$ A1

A.1 Discretisation of the momentum equation . . . . . . . . . . . A1

A.1.1 Transient term . . . . . . . . . . . . . A2

A.1.2 Convection term . . . . . . . . . . . . . A3

A.1.3 Diffusion term . . . . . . . . . . . . . . A4

A.1.4 Source term . . . . . . . . . . . . A A5

A.1.5 Convection and diffusion terms . . . . . . . . . . . A6

A.1.6 Rhie - Chow interpolation method . . . . . . . . . . . . A A6

A.1.6.1 Implementation in OpenFoam . . . . . . . . . . . . A10

$\begin{array}{ll}\text { Appendix A: References } & \text { A12 }\end{array}$

$\begin{array}{ll}\text { Appendix B: OpenFoam code } & \text { B1 }\end{array}$ 
B.1 "boussinesqBuoyantFoam" Solver . . . . . . . . . . . . . . . . . B1

Appendix C: Data attachment $\quad$ C1

C.1 Overview . . . . . . . . . . . . . . . . . . . C1

C.2 Summary of directory structure . . . . . . . . . . . . C1

Appendix C: References $\quad$ C3 


\section{List of figures}

Figure 1.1: A typical finned heat sink [1] . . . . . . . . . . . . . 2

Figure 1.2: $\quad$ Microprocessor transistor counts 1971-2011 and Moore's Law. [3] 3

Figure 3.1: The PISO algorithm. . . . . . . . . . . . . . . . . 23

Figure 3.2: Self driven cavity problem. . . . . . . . . . . . . . . . . 25

Figure 3.3: Heated parallel plates cooled by natural convection. . . . . . . 27

Figure 3.4: The effect of the plate-to-plate spacing on the heat transfer density rate for $\operatorname{Pr}=0.72 \ldots \ldots \ldots 27$

Figure 4.1: Parametrisation routine for OpenFoam . . . . . . . . . . . . . 32

Figure 4.2: Contour representation of a constrained problem [55] . . . . . . 34

Figure 4.3: Illustration of a maximisation function versus a minimisation function [55]. . . . . . . . . . . . . . . . . . . . . . . . . . 34

Figure 4.4: Optimisation routine for OpenFoam. . . . . . . . . . . . . 36

Figure 5.1: $\quad$ Single row of counter-rotating cylinders in natural convection. . 39

Figure 5.2: The computational domain and boundary conditions for a set of counter-rotating cylinders. . . . . . . . . . . . . . 40

Figure 5.3: Multi scale row of counter-rotating cylinders in natural convection. 42

Figure 5.4: The computational domain and boundary conditions for a set of counter-rotating cylinders. . . . . . . . . . . . . . . 43

Figure 5.5: Illustration of the discretised flow domain. . . . . . . . . . 47

Figure 6.1: Illustration of the thermal boundary layer around a stationary cylinder . . . . . . . . . . . . . . . . . . 52

Figure 6.2: The maximization of the heat transfer density rate for the assemble shown in Fig. 5.1 for $R a=10^{3}, \operatorname{Pr}=0.72$ and $\tilde{\omega}_{0}=0$.

Figure 6.3: The optimal cylinder-to-cylinder spacings and corresponding heat transfer density rates for a row of cylinders shown in Fig. 5.1 for $\operatorname{Pr}=0.71, \tilde{\omega}_{0}=0$ and $10^{1} \leq R a \leq 10^{4} \ldots \ldots . . .54$ 
Figure 6.4: The maximization of the heat transfer density rate for the cylinders shown in Fig. 5.1 for $R a=10^{1}$ and $P r=0.71$ at different rotation speeds.

Figure 6.5: The optimal cylinder-to-cylinder spacings for a row of rotating cylinders shown in Fig. 5.1 for $\operatorname{Pr}=0.71 \ldots$. . . . . . . . .

Figure 6.6: The maximum heat transfer density rates for a row of rotating cylinders shown in Fig. 5.1 for $\operatorname{Pr}=0.71 \ldots$. . . . . . . .

Figure 6.7: The effect of cylinder rotational speed on the thermal boundary layer for a row of rotating cylinders shown in Fig. 5.1 . . . . .

Figure 6.8: Contour plot of the heat transfer density rates for a row of rotating cylinders shown in Fig. 5.3 for $R a=10^{3}$ and $\operatorname{Pr}=0.71 .60$

Figure 6.9: The maximum heat transfer density rates for a row of rotating cylinders shown in Fig. 5.3 for $R a=10^{3}$ and $\operatorname{Pr}=0.71$. . . .

Figure 6.10: The optimal cylinder-to-cylinder spacings and corresponding heat transfer density rates for a row of cylinders shown in Fig. 5.3 for $\operatorname{Pr}=0.72, \tilde{\omega}_{0}=0$ and $10^{2} \leq R a \leq 10^{4}$. . . . . . . .

Figure 6.11: The optimal cylinder-to-cylinder spacings and small cylinder diameter for a row of rotating cylinders shown in Fig. 5.3 for $\operatorname{Pr}=0.72 \ldots \ldots \ldots \ldots \ldots \ldots \ldots$

Figure 6.12: The maximum heat transfer density rates for a row of rotating cylinders shown in Fig. 5.3 for $\operatorname{Pr}=0.72 \ldots$. . . . . . . . .

Figure 6.13: The effect of increasing of cylinder rotational speed and the effect of increasing the complexity on the optimal cylinder-tocylinder spacing for $\operatorname{Pr}=0.72 \ldots \ldots \ldots$. . . . . . . .

Figure 6.14: The effect of increasing of cylinder rotational speed and the effect of increasing the complexity on the maximum heat transfer density rate for $\operatorname{Pr}=0.72 \ldots \ldots \ldots$. . . . . . . .

Figure 6.15: The effect of cylinder rotational speed on the thermal boundary layer for a multi scale structure of rotating cylinders shown in

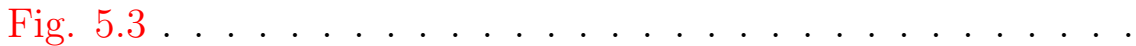

Figure 7.1: $\quad$ Single row of counter-rotating cylinders in natural convection with multiple cylinder-to-cylinder spacings. . . . . . . . . . . .

Figure 7.2: Multi scale row of counter-rotating cylinders in natural convection with multiple cylinder-to-cylinder spacings. . . . . . . . . 
Figure A.1: One dimensional control volume. . . . . . . . . . . . . . . . A7 


\section{List of tables}

Table 3.1: Heat transfer rate, per unit length, from the heated side of the cavity to the cooled side of the cavity for $\operatorname{Pr}=0.72 \ldots 26$

Table 5.1: $\quad$ Single scale mesh refinement summary: number of elements per unit length at each Rayleigh number $\left(\operatorname{Pr}=0.72\right.$ and $\left.\tilde{\omega}_{0}=0\right) . \quad . \quad 48$

Table 5.2: Single scale mesh refinement study for $R a=10^{3}\left(\tilde{H}_{u}=1, \tilde{H}_{d}=\right.$ $2, \tilde{S}_{0}=0.3, \tilde{\omega}_{0}=0$ and $\left.\operatorname{Pr}=0.72\right) \ldots \ldots \ldots$.

Table 5.3: Single scale domain size summary: upstream and downstream lengths at each Rayleigh number $\left(\operatorname{Pr}=0.72\right.$ and $\left.\tilde{\omega}_{0}=0\right) \ldots$. .

Table 5.4: Multi scale mesh refinement summary: number of elements per unit length at each Rayleigh number $\left(\operatorname{Pr}=0.72\right.$ and $\left.\tilde{\omega}_{0}=0\right)$. .

Table 5.5: Multi scale mesh refinement study for $R a=10^{3}\left(\tilde{H}_{u}=0.5\right.$, $\tilde{H}_{d}=3.5, \tilde{S}_{0}=0.5, \tilde{\omega}_{0}=0$ and $\left.\operatorname{Pr}=0.72\right) \ldots \ldots \ldots$

Table 5.6: Multi scale domain size summary: upstream and downstream lengths at each Rayleigh number $\left(\operatorname{Pr}=0.72\right.$ and $\left.\tilde{\omega}_{0}=0\right) \ldots$.

Table 6.1: $\quad$ Scale analysis for the optimal cylinder-to-cylinder spacing for a single scale structure. . . . . . . . . . . . . . . . . .

Table 6.2: Coefficients for the power law correlation of the form $C R a^{n}$ for $\tilde{S}_{0, o p t}$ and $\tilde{q}_{m} \ldots \ldots \ldots \ldots \ldots \ldots$ 


\section{Nomenclature}

\section{Latin symbols}

A Area

$m^{2}$

$C_{p} \quad$ Specific heat capacity

$J / k g K$

$d i \quad$ Number of large diameter cylinders

dj $\quad$ Number of small diameter cylinders

$D_{0} \quad$ Large cylinder diameter

$m$

$D_{1} \quad$ Small cylinder diameter

$m$

$f(\mathbf{x}) \quad$ Objective function

$\mathbf{F}_{\text {buoyant }}$ Buoyancy body force vector

$N / m^{3}$

$g(\mathbf{x}) \quad$ Inequality constraint function

g Gravitational acceleration vector

$\mathrm{m} / \mathrm{s}^{2}$

$h \quad$ Convective heat transfer coefficient

$W / m^{2} K$

$h(\mathbf{x}) \quad$ Equality constraint function

$H \quad$ Enthalpy

$J / k g$

$H_{d} \quad$ Downstream flow length

$m$

$H_{u} \quad$ Upstream flow length

$m$

$k \quad$ Thermal conductivity

$W / m K$ 
$\hat{k} \quad$ Unit vector

L Unit length

$m$

$m \quad$ Number of inequality constraints

$P \quad$ Pressure

$P a$

$P_{d} \quad$ Dynamic pressure

$P a$

$P_{\text {ref }} \quad$ Reference pressure

$P a$

$P_{s} \quad$ Static pressure

$P a$

$P_{t} \quad$ Total pressure

$P a$

$q \quad$ Total heat transfer rate

W

$q^{\prime} \quad$ Heat transfer rate per unit length

$q^{\prime \prime} \quad$ Heat transfer rate per unit area

$W / m^{2}$

$q^{\prime \prime \prime} \quad$ Heat transfer density rate

$W / m^{3}$

$r \quad$ Number of equality constraints

$R \quad$ Solution residual

$S_{0} \quad$ Spacing between large cylinders

$m$

$t \quad$ Time / Time step

$s$

T $\quad$ Fluid temperature

$K$

$T_{c} \quad$ Cold wall temperature $\quad K$

$T_{h} \quad$ Hot wall temperature $\quad K$

$T_{w} \quad$ Wall temperature $\quad K$

$T_{\infty} \quad$ Inlet fluid temperature $\quad K$

$u, v, w \quad$ Velocity components $\mathrm{m} / \mathrm{s}$

U Velocity vector $\mathrm{m} / \mathrm{s}$ 
W Assembly width

$x \quad$ Design variable

x Design variable vector

$x, y, z \quad$ Cartesian coordinates

\section{Greek symbols}

$\alpha \quad$ Thermal diffusivity

$\beta \quad$ Thermal expansion coefficient

$\delta_{i j} \quad$ Kronecker delta function

$\delta_{T} \quad$ Thermal boundary layer thickness

$\lambda \quad$ Coefficient of bulk viscosity

$\mu \quad$ Viscosity

$\mathrm{kg} / \mathrm{ms}$

$\nu \quad$ Kinematic viscosity

$m^{2} / s$

$\rho \quad$ Fluid density

$\mathrm{kg} / \mathrm{m}^{3}$

$\rho_{o} \quad$ Fluid reference density

$\mathrm{kg} / \mathrm{m}^{3}$

$\rho^{\prime} \quad$ Density change relative to reference density

$\mathrm{kg} / \mathrm{m}^{3}$

$\Phi \quad$ Dissipation function

$W / m^{3}$

$\omega_{0} \quad$ Cylinder angular velocity

$\mathrm{rad} / \mathrm{s}$

\section{Non-dimensional numbers}

$\tilde{L} \quad$ Characteristic Length

$N u \quad$ Nusselt number

$\operatorname{Pr} \quad$ Prandtl number

$\tilde{q} \quad$ Dimensionless heat transfer density rate

$R a \quad$ Rayleigh number 


\section{Subscripts}

$[1],[2] \quad$ number of length scales in the flow configuration

$i, j, n \quad$ Numerical indices

$m \quad$ Maximum

$\max \quad$ Maximisation function

min Minimisation function

sn In the direction of the surface normal vector

opt Optimum

$t \quad$ Time step

\section{Superscripts}

$0, i, k \quad$ Iteration indices

$n \quad$ Numerical index

T Transpose

* $\quad$ Optimum design variable vector

\section{Accents}

r Dimensionless variables 


\section{Chapter 1}

\section{Introduction}

\section{$1.1 \quad$ Background}

Efficiency is a key aspect in design, which has become prevalent in the design of heat transfer devices such as heat sinks and pin fins. Research has been and is still being conducted on this subject with the aim of extracting more and more heat from a given space through the maximization of the packing of heat-generating material per unit volume. This drive to augment heat transfer devices has become reinforced by modern electronic systems which produce high amounts of heat due to the ever increasing power-to-volume ratio employed in such systems.

Heat transfer devices, such as heat sinks (shown in Fig. 1.1), work by removing heat from a given system, with which they are in direct contact, and transferring that extracted heat to a working or surrounding fluid. The mechanisms through which heat is transferred are namely:

- Conduction: heat is transferred when neighbouring atoms vibrate against each other, or as electrons move from one atom to another

- Convection: heat is transferred from an object to its surroundings through the movement of fluid 
- Radiation: specifically thermal radiation is radiative energy carried by electromagnetic waves, which differs from conduction and convection in that it require no medium for their propagation.

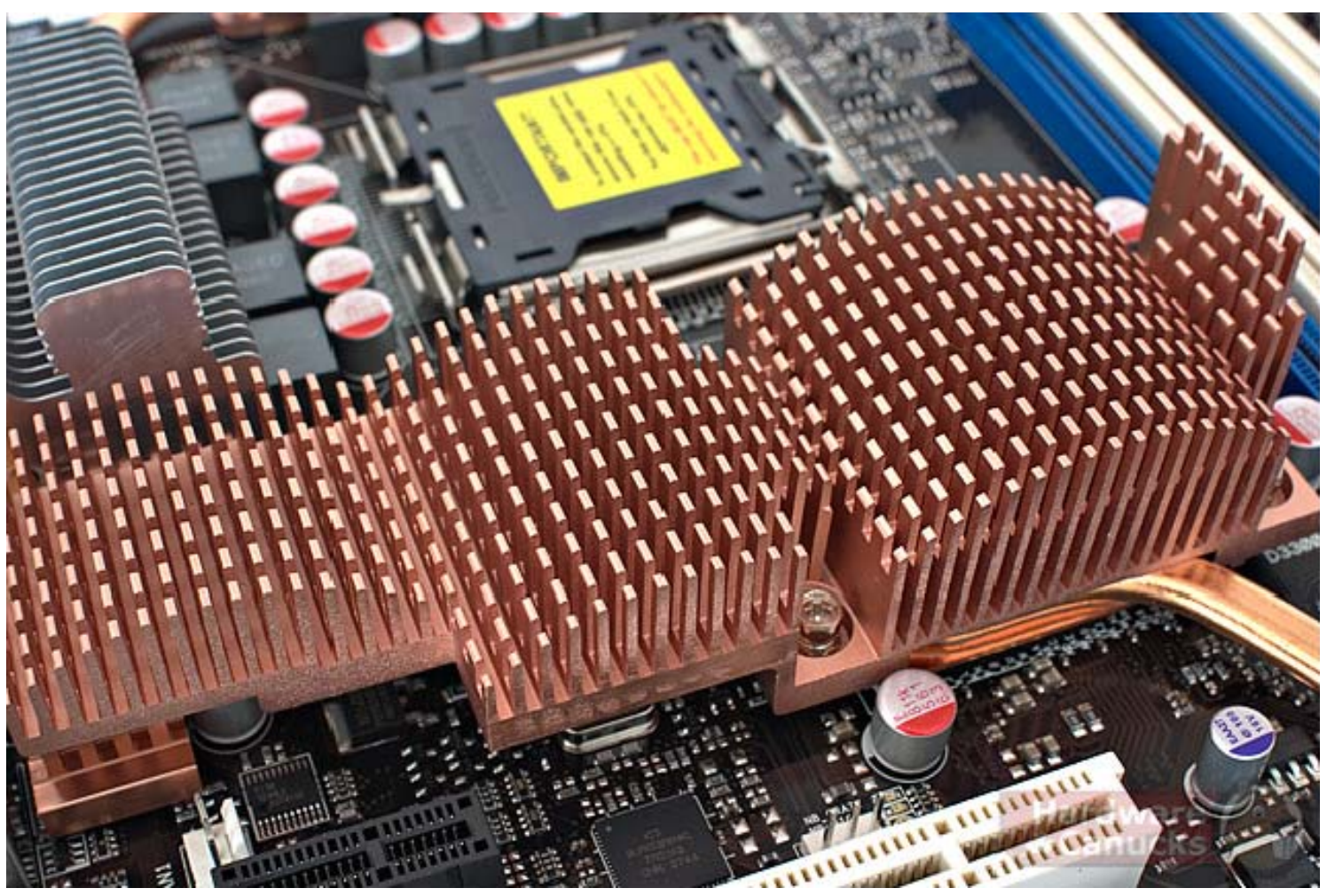

Figure 1.1: A typical finned heat sink [1]

The rapid development of electronic and electrical systems has increased the thermal management requirements of these systems. In 1965 the Intel co-founder Gordon Moore made a empirical observation, regarding the rapid improvement of integrated electronics: "The complexity for minimum component costs has increased at a rate of roughly a factor of two per year... Certainly over the short term this rate can be expected to continue, if not to increase. Over the longer term, the rate of increase is a bit more uncertain, although there is no reason to believe it will not remain nearly constant for at least 10 years. That means by 1975, the number of components per integrated circuit for minimum cost will be 65,000. I believe that such a large circuit can be built on a single wafer" [2]. This prediction in now more commonly know as Moore's Law which states that transistor density on integrated circuits doubles about every two years due to the lowering of the minimum manufacturing cost per component each year (shown in Fig. 1.2). 


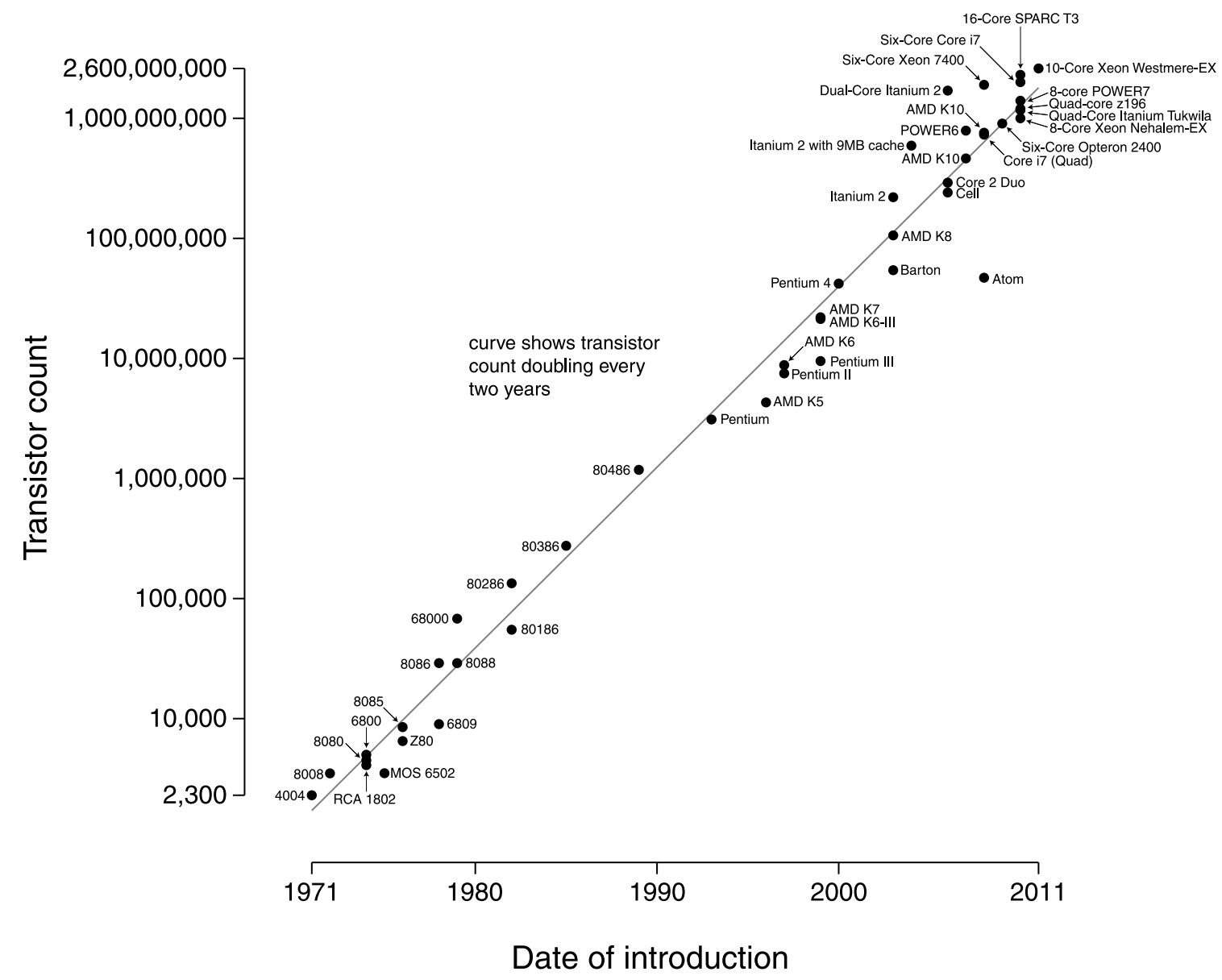

Figure 1.2: Microprocessor transistor counts 1971-2011 and Moore's Law. [3]

\section{What is natural convection?}

Natural Convection is a heat transfer mechanism in which the fluid motion, of a working or ambient fluid, is not generated by external sources (like a pump or fan) but rather by the changes in the body forces of the fluid, caused due to the presents of gravity. The changes in fluid body forces are cause by density differences which in turn are created by temperature gradients in the fluid. In natural convection, the fluid surrounding a heat source receives heat, becomes less dense and rises. The surrounding cooler fluid then moves in to replace it. This cooler fluid is then in-turn heated and the process continues. The fluid density due to the energy exchange can be expressed in terms of the coefficient of thermal expansion, $\beta$, given as:

$$
\rho=\rho_{o}(1-\beta \Delta T)
$$


where $\rho_{o}$ is the bulk fluid density, $\rho$ is the fluid density inside the heated layer, and $\Delta T$ is the temperature difference between the heated fluid and the bulk of free stream fluid. The body force, specifically the buoyant force per unit volume $\left(\mathbf{F}_{\text {buoyant }}\right)$, due to the density changes can be expressed as [4]:

$$
\mathbf{F}_{\text {buoyant }}=\left(\rho_{o}-\rho\right) \mathbf{g}
$$

Upon substituting Eq. 1.2 into Eq. 1.1, the buoyant force becomes:

$$
\mathbf{F}_{\text {buoyant }}=\rho_{o} \mathbf{g} \beta \Delta T
$$

where $\mathbf{g}$ is the gravitational acceleration vector.

The heat transfer rate equation for convection was first expressed by Newton in 1701, and is referred to as Newton's "law" of cooling. This equation is:

$$
q=h A \Delta T
$$

where $q$ is the rate of convective heat transfer, $A$ is the area normal to the direction of heat flow, $h$ is the convective heat transfer coefficient and $\Delta T$ is the temperature difference between the surface and the bulk or free stream fluid. Equation 1.4 is not a law but a definition of the coefficient $h$.

At the surface of any heat source there exists a hydrodynamic boundary layer that plays an important role in heat transfer. Near the surface of the heat source, the heat is transferred to the fluid through conduction. The equation describing this mechanism was first stated in 1822 by Fourier, also known as Fourier's first law of heat conduction, and is given as:

$$
q=k A \frac{\Delta T}{\delta x}
$$

where $k$ is the thermal conductivity and $\Delta T$ is the temperature difference between the surface and the surrounding heated fluid. Equation 1.4 and Eq. 1.5 can be combined and non-dimensionalised to give:

$$
N u=\frac{h L}{k}
$$

where $L$ is the characteristic length and the Nusselt number $N u$ is the ratio of the temperature gradient at the surface to an overall or reference temperature gradient. 
Why do research in natural convection?

The most common reason for selecting natural convection over forced convection is in order to eliminate the pump or fan from the heat transfer device or system. Pumps and fans introduce additional points of failure and also increase the noise of the system. More importantly pumps and fans also require additional power input into the system whereas natural convection is a self sustained mechanism, requiring no additional power input, that provides a continuous heat transfer rate.

The disadvantage of natural convection over forced convection is that the convective heat transfer coefficient is considerably lower for natural convection systems compared to that achievable by forced convection systems [5]. Subsequently, for example in the design of heat sinks, a larger surface area or footprint of the heat sink is required to achieve the equivalent performance of a forced convection heat sink, cf. Eq. 1.4; thus research into optimal packing is beneficial.

Smarter solutions, utilizing either mixed convection or an alternative use of power input into the system, are needed in order to bridge the gap between pure natural convection and forced convection systems; thereby creating a sort of enhanced natural convection system. These such systems can utilize the functionality of pure natural convection whist the heat source is operating at a lower capacity and then utilize the enhancement capability when the heat source is operating at a higher or maximum capacity. This type of heat transfer system has the potential to reduce the overall power input into system while maintaining the required heat transfer rates. An example of such a heat source would be a micro-processor. This thesis thus presents one such possible solution by investigating the effect of rotating heat generating cylinders cooled by natural convection.

Examples of the practical application of heat sinks, designed to utilize natural convection, used to cool electronic components can be found in: LED lighting, solid state thermoelectric Peltier modules for compact refrigerators, Ball Grid Arrays (BGA) and power-line transformers. Examples of heat transfer from a rotating heat generating solid to a surrounding fluid can be found in: the paper, rubber and plastic, and textile industries (steam, hot oil or induction heated rollers) and a wide variety of rotating machinery. 


\section{$1.2 \quad$ Research objectives}

The objectives of the research presented in this thesis are:

- to numerically investigate the thermal behaviour of an assembly of consecutive rotating cylinders cooled by natural convection,

- to geometrically optimise this assembly for single scale and multi scale configurations with the objective of maximising the heat transfer density rate (heat transfer per unit volume),

- to couple and automate the numerical computation with a mathematical optimisation algorithm through the use of programming,

- to report all results in the form of non-dimensional quantities (Rayleigh number, Prandtl Number, etc.) and establish feasible correlations between:

- the Rayleigh number, cylinder rotation speed and heat transfer density rate and

- the Rayleigh number, cylinder rotation speed and optimal geometric configuration.

\subsection{Scope of study}

A multidisciplinary approach is employed in this thesis combining mathematical optimisation, computation fluid dynamics and programming to efficiently optimise the geometric configuration of an assembly of consecutive rotating cylinders cooled by natural convection. The fluid flow characteristics are limited to the two dimensional, steady-state, laminar and incompressible flow regime with the Boussinesq approximation assumed valid for all cases. The flow regime considered is limited to a Rayleigh number range of $10^{1} \leq R a \leq 10^{4}$ with a fixed Prandtl number of $\operatorname{Pr}=0.72$. The non-dimensional cylinder rotation speed is limited to a range of $0 \leq \tilde{\omega}_{0} \leq 10$. An automated optimisation routine is utilized for design cases with two or more design variables subject to various constraints. 


\subsection{Structure of thesis}

This thesis is divided into chapters for better organisation and ease of reading. This thesis therefore consists of the following chapters:

- Chapter 2 gives insight into relevant published work on heat transfer devices specifically relating to optimal packing and heat transfer density rate. This chapter also highlights the effect rotation has on a cylinder with respects to the heat transfer rate.

- Chapter 3 gives an overview of the numerical modelling techniques as well as the numerical methods used in solving the computational model. The mass, momentum and energy conservation equations governing the fluid flow and heat transfer characteristics are discussed. The techniques used in treating the pressurevelocity coupling (in the momentum equation) is also presented, with additional information given in Appendix A and Appendix B.

- Chapter 4 presents the tools and methods used to automate the entire computational process for a selected geometric variable range (Parameterisation). The method in which this automated process is then coupled to an optimisation algorithm is also discussed. Finally a brief overview of the principals of optimisation and the governing equations of the optimisation algorithm are highlighted. The the programming scripts and details thereof are attached in electronic form in Appendix C.

- Chapter 5 applies the methods presented in Chapter 3 to the numerical model for single scale and multi scale geometry configurations. The governing mass, momentum and energy conservation equations are non-dimensionalised in terms of Rayleigh number and Prandtl number and the quantity used, as the objective function, to evaluate the geometric configurations is discussed. The convergence criteria of the CFD solution scheme as well as the optimisation algorithm is also discussed.

- Chapter 6 opens by presenting the expected results from the numerical model, presented in Chapter 5, through the use of scale analysis. The results from the numerical model simulations are then discussed in depth and compared to the scale analysis approximations. Correlations between the Rayleigh number, 
cylinder rotation speed and heat transfer density rate as well as the Rayleigh number, cylinder rotation speed and optimal geometric configuration are also presented.

- Chapter 7 provides a summary of the results, conclusions that may be drawn from these results and recommendations for future work. 


\section{Chapter 2}

\section{Literature study}

\section{$2.1 \quad$ Introduction}

The strive for greater heat transfer density rates has been the driving force behind many of the miniaturization efforts, augmentations and unconventional ways of designing heat transfer devices. It is the purpose of this thesis to maximize the heat transfer density rate of a row of heat-generating rotating cylinders in steady laminar singlephase natural convection. This chapter starts by giving an overview of constructal theory and design, as a method for designing optimal geometric space-constrained flow configurations, in Section 2.2. Previous research conducted in optimizing heat transfer devices is then presented in Section 2.3. Previous research into the effect of increasing the complexity of the geometric structure of the heat transfer devices in then presented in Section 2.3.1. Finally, previous research into the effect of cylinder rotation in various flow regimes is presented in Section 2.4.

\subsection{Constructal flow geometry}

One method for designing optimal geometric space-constrained flow configurations is constructal theory and design [5-8]. According to this method, the flow configuration is free to morph (change) in the pursuit of maximising global performance subject to 
global constraints. The resulting optimal (constructal) configuration is determined or evaluated, not assumed.

Many examples of constructal-design configurations for determining the optimal internal spacing for heat-generating volumes cooled by plates, and pin fins will be discussed below in Section 2.3. In each case, the total volume is fixed and the objective is to maximize the global heat transfer from the heat-generating solid to the surrounding fluid of the package (or the volume-averaged heat transfer density rate). The result is a single length scale: the optimal spacing, and this length scale is distributed uniformly throughout the volume.

This thesis focuses on taking the constructal design of optimal spacings in a new direction by numerically formulating the problem (Chapter 3) and mathematically optimising the flow configuration (Chapter 4). An optimized single scale and multi scale flow structure (subject to global constraints), that achieves even higher levels of heat transfer density rate, is sought for each flow regime (Rayleigh number and cylinder rotation speed). The process of optimising the flow configuration is often one of "brute force", whereby the design space is broken up into a finite number of points at which the global objective is evaluated. By introducing a mathematical optimization routine, a seemingly infinite design space can be evaluated in order to obtain the optimal flow configuration.

\subsection{Heat transfer devices}

This strive for greater heat transfer density rates has lead researchers to study the optimized geometric configurations for various architectures such as: the optimal spacing of parallel plates and the optimal spacing of cylinders for various flow regimes.

Bejan and Sciubba [9] reported the optimal board-to-board spacing and maximum total heat transfer rate from a stack of parallel boards cooled by laminar forced convection. In a bid to maximise the total heat transfer rate, an order-of-magnitude analysis, together with the intersecting of asymptotes method, was employed to develop exact solutions for both uniform temperature and uniform heat flux boundary conditions. It was learnt that the optimal spacing is proportional to the distance between the 
channels to the power of $1 / 2$ and the pressure head maintained across the stack to the power of $-1 / 4$.

Similarly to Ref [9], Bejan and Morega [10] reported the optimal plate-to-plate spacing when the stack is cooled by turbulent forced convection. It was shown that the optimal spacing not only depends on the pressure difference number, but it was also very sensitive to two parameters: the Prandtl Number and the ratio of plate thickness to plate length. It is also reported that the optimal spacing increases as these two parameters increase.

Da Silva et al. [11] showed numerically that the entire flow geometry of a vertical diverging or converging channel with laminar natural convection could be optimized for maximal heat transfer density rate. The geometry was free to change in three ways: (1) the spacing between the walls, (2) the distribution of heating along the walls, and (3) the angle between the two walls. It was reported that the use of all of the available heat transfer surface was the best heating distribution along the walls of the channel. Further, it was reported that a diverging channel had some improvement on maximising the total heat transfer density rate for small angles of divergence. At larger angles of divergence numerical instability became prevalent and convergence of the solution could not be reached. It was further shown, through scale analysis, that parallel or near parallel walls were the best solution from the point of view of maximising the total heat transfer density rate.

Bello-Ochende and Bejan [12] presented the optimal geometric spacing between parallel plates for two flow regime limits: pure natural convection and pure forced convection. The in-between flow regime of mixed convection is also presented. It was shown that the numerical results match the results in the limits of natural convection $[13,14]$ and forced convection [9]. The authors further reported a correlation for the mixed convection regime as well as a single formula for the optimal plate-to-plate spacing for the entire flow domain (from natural convection through to forced convection).

Stanescu et al. [15] carried out experimental, numerical and analytical studies of the optimal spacing between cylinders, in a bundle, cooled by cross-flow forced convection. The cylinder array occupied a fixed volume and was exposed to a free stream velocity and temperature with the objective maximizing the overall heat transfer rate between all the cylinders and the free stream. It was shown that the optimal cylinder-to-cylinder 
spacing decreases as the free stream velocity increase, and as the flow length of the array decreases.

Similar to the work in Ref [15], Stanescu et al. [16] also carried out experimental, numerical and analytical studies of the optimal spacing between cylinders, in a array, cooled by laminar natural convection. It was shown that the optimal cylinder-tocylinder spacing was relatively insensitive to the wall boundary condition (isothermal or uniform heat flux). It was also reported that the optimal spacing decreases as the Rayleigh number increases.

\subsubsection{Increased geometric complexity}

The effect of increasing the complexity of the geometry, by adding additional length scales to the flow configuration, follows the principals of constructal theory and design. The key to improved performance in heat transfer density rates is more flexibility or freedom to change. Thus the addition of more length scales to the flow configuration creates more degrees of freedom, i.e. more directions for the flow configuration to morph.

Bello-Ochende and Bejan [17] considered the multi scale structure: parallel plates with multiple length scales in forced convection. Progressively smaller heat generating plates were inserted into the wedge-shaped region, at the entrance of the flow domain, to create a multi scale structure. Optimal spacings were reported for structures with one, two and three length scales. It was shown that the performance increases as the geometric complexity increases, but a diminishing return are also observed. It was also shown that the optimized spacings increased slightly with each new (smaller) plate that was inserted in the entrance region of each channel. The results report in this literature confirmed the results, derived analytically by Bejan et al. [18], for the same problem. A three dimensional parallel plate multi scale structure cooled by laminar natural convection was also considered, more recently, by Bello-Ochende et al. [19].

Similar to the work done in Ref [17], da Silva and Bejan [20] considered the parallel plate multi scale structure cooled by natural convection. Similar results were reported in that the maximized heat transfer density increases as the optimized complexity of the flow structure increases. It was also shown that there is a smallest (cut-off) length 
scale below which the boundary layers are no longer distinct, and where the sequence of generating optimal length scales ends.

Bello-Ochende and Bejan [21] considered the multi scale structure of a row of parallel cylinder cooled by laminar forced convection. Smaller cylinders were placed at the entrance to the assembly, in the wedge-shaped flow regions occupied by fluid that had not yet been used for heat transfer. The optimized flow configuration and performance for structures with 1,2 and 4 degrees of freedom were reported. It was reported that the total heat transfer density rate increased (with diminishing return) as the optimized structure became more complex. It was also shown that the optimized cylinder diameters were relatively robust (insensitive to changes in complexity and pressure difference) and the optimized spacings decreased monotonically as the driving pressure difference increased.

Similar to the work done in Ref [21], Bello-Ochende and Bejan [22] also considered the multi scale structure of a row of parallel heat-generating cylinders cooled by natural convection. The results reported were similar to the results in Ref [21] in that the optimized cylinder diameters were relatively robust (insensitive to changes in complexity and Rayleigh number) and the optimized spacings decreased monotonically as the Rayleigh number increased. For a single scale structure the optimal spacings and corresponding heat transfer density rates, for each flow regime, were correlated by the power law:

$$
\begin{aligned}
\frac{S_{0[1] \text { opt }}}{D_{0}} & =1.32 R a^{-0.22} \\
\tilde{q}_{[1] m} & =0.65 R a^{0.30}
\end{aligned}
$$

For a multi scale structure, with two length scales, the optimal spacings and corresponding heat transfer density rates, for each flow regime, were correlated by the power law:

$$
\begin{aligned}
\frac{S_{0[2] o p t}}{D_{0}} & =2.22 R a^{-0.22} \\
\tilde{q}_{[2] m} & =0.85 R a^{0.30}
\end{aligned}
$$

where the subscripts 1 and 2 refer to the number of length scales in the flow configuration. The optimal small cylinder diameter (for two length scales) was reported to be 
very robust for all flow regimes and was found to be:

$$
\frac{D_{1}}{D_{0}}=0.2
$$

The results reported by Bello-Ochende and Bejan [22] are the cornerstone or benchmark on which the work of this thesis is based and as such will be used as a reference. This thesis builds onto this literature by considering the effect of cylinder rotation speed on the optimised flow configuration and the total heat transfer density rate.

\subsection{The effect of rotation}

A single stationary cylinder in steady state convection will have a developed thermal and momentum boundary layer and a corresponding heat transfer rate (for a given flow regime). The effect of rotation on this cylinder will thus change the behaviour (shape) of the developed boundary layers, which in turn effects the heat transfer rate and fluid flow around this cylinder. The heat transfer rate and fluid flow around a single rotating cylinder has been studied previously by numerous authors.

Badr and Dennis [23] considered the problem of laminar forced convective heat transfer from an isothermal circular cylinder rotating about its own axis located in a uniform stream. The authors reported that the temperature fields are strongly influenced by the rotational speed of the cylinder and contradictory to expectation they found that the overall heat transfer coefficient tends to decrease as the rotational of the cylinder increases. They attributed this to the presences of a rotating fluid layer around the cylinder that separates the cylinder from the main flow stream.

Chiou and Lee [24] considered a problem of forced convection on a rotating cylinder cooled with an air jet. The results confirmed that the overall heat transfer is enhanced at lower rotational speeds and at higher rotational speeds the effect became reversed. They attributed this to the presences of a layer of dead air around the cylinder.

Panda and Chhabra [25] considered a problem of forced convection heat transfer from a heated cylinder rotating in streaming power-law fluids. The results show a similar behaviour of the heat transfer rate: for moderate rotational velocities at low 
Reynolds numbers the heat transfer rate is enhanced and there is an envelope of conditions (Reynolds number, rotation speed and power-law index) in which rotation has a negative effect on the heat transfer rate.

Similar literature includes the works of Gshwendtner [26], Mohanty et al. [27], Oesterle et al. [28], Ozerdem [29], Paramane and Sharma [30, 31], Yan and Zu [32] and Nobari et al. [33].

More recent studies have been conducted by Joucaviel et al. [34], with a single scale structure of a row of heat-generating rotating cylinders cooled by forced convection. The authors reported that the effect of rotation was beneficial from the point of view of maximising the heat transfer density rate. The results also showed that a counterrotation configuration increases the heat transfer density rate more efficiently when compared to a co-rotation configuration. In fact the authors states that a co-rotating configuration "seems to be useless", in that the heat transfer density rate decreases and the optimal spacing increases with the increase in rotational speed.

Bello-Ochende et al. [35] then built onto the work of Joucaviel et al. [34] by considering the effect of rotation on a multi scale configuration as well as the placement of the small cylinder. It was reported that optimal small cylinder placement is such that all cylinders are aligned along their leading edges.

\subsection{Conclusion}

This chapter provided an overview of some of the available literature on the designing of heat transfer devices with the objective of enhancing the volume-averaged heat transfer density rate. The published work includes theoretical analysis, experimental procedures and numerical modelling, which are used to determine the optimal structure. On overview of the literature showing the effect of rotation on a single cylinder and on a row of consecutive was also presented. 


\section{Chapter 3}

\section{Numerical modelling}

\subsection{Introduction}

This chapter gives an overview of the numerical methods used for modelling and solving a typical engineering problem and how these methods are applied in a Computational Fluid Dynamics (CFD) package or code (Section 3.2). The governing equations that characterise the fluid flow and heat transfer are also discussed (Section 3.4). An overview of the Finite Volume discretisation Method (FVM) (Section 3.5.1) and PISO algorithm (Section 3.5.2), used for solving the governing equations, is also presented. Finally the verification of these numerical methods, as implemented in OpenFOAM, is put foreward (Section 3.6).

\subsection{Overview of numerical modelling}

In recent times numerical modelling has been made easier and readily accessible due to the advances and accessibility of the personal computer as well as the development of CFD packages or codes structured around numerous numerical algorithms. This advancement has lead to easier and faster solutions for fluid flow and heat transfer problems in engineering. The solutions for fluid flow and heat transfer problems through the use of a CFD package or code involves three stages [36], namely: 
1. Pre-processing, which involves defining a computation domain. The computation domain is first broken up the into smaller, non-overlapping, pieces or sub-domains (mesh generation) and then the relevant boundary conditions and fluid properties are defined for the domain.

2. Solution, which involves the solution of the governing equations at each of the smaller pieces or volumes (making up the computation domain) through the use of numerical approximation methods (finite difference, finite volume, finite element).

3. Post-processing, which involves the collection and evaluation of results and data. At this stage the user is also often equipped with visualisation tools such as contour plots, stream-line plots, particle tracking etc.

\subsection{Geometry and mesh generation}

Mesh generation is an integral part of the pre-processing stage when using a CFD package or code. This process involves generating the geometry of the computational domain or simply stated the region of interest (the fluid and / or material volume). This domain is then broken up into a finite number of discrete sub-domains, called control volumes or cells. The boundary conditions are then applied to the computational domain. This disrectisation of the the computational domain produces a numerical description to the solution domain, including the spacial and temporal locations at which the solution to the flow variables are sought.

This disrectisation process has been made easier in recent times through the use of commercial automated mesh generators in which the user can, with the help of a Graphical User Interface (GUI), generate meshes by the click of a mouse. Unfortunately many open-source packages or codes are still based on a "text-file" input method whereby the user has to manually input all required information into a "text-file" prior to executing the mesh generator. This such "text-file" based input method used for mesh generation is employed as a build-in function in OpenFOAM (an open source finite volume CFD package) [37]. The method adopted, in this thesis, for generating a mesh through the use of OpenFOAM consists of the following steps [38]: 
1. Structure the computation domain, in which the computational domain is broken up into smaller volumes, each of which forming a quadrilaterally-faced hexahedron (this is done in order to obtain a structured hexahedral mesh).

2. Determine all vertex coordinates and indices of the hexahedra volumes and input this information into a text-file.

3. Determine each hexahedron volume connectivity, based on the vertex indices and input this information into the text-file.

4. Determine the number of elements as well as the element grading in each direction of the hexahedron volume local coordinate system, for all the hexahedra volumes, and input this information into the text-file.

5. Determine all sets of vertex indices (forming a face of a hexahedron volume lying on a boundary) for each computational boundary and input this information into the text-file.

6. Execute OpenFOAM's build-in mesh generation function, "blockMesh".

The advantage of this method is that the user can take advantage of it through the use of a programming language (e.g. Octave [39], Python [40], etc.) and thereby, after initially setting up one case file, automate the mesh generation process.

\subsection{Governing equations}

The governing equations are a set of non-linear partial differential equations that describe the fluid flow and heat transfer characteristics of a typical engineering problem. The equations that govern these characteristics are the continuity (conservation of mass), momentum and energy equations. 


\subsubsection{Conservation of mass}

The general form of the continuity equation, in an Eulerian specification of the fluid flow field, is given by [41]:

$$
\frac{\partial \rho}{\partial t}+\rho \operatorname{div}(\mathbf{U})=0
$$

where $\rho$ is the density of the fluid, $t$ is the time and $\mathbf{U}$ is the velocity vector field of the fluid. For steady-state incompressible (constant density) flow, Equation 3.1 simplifies to:

$$
\operatorname{div}(\mathbf{U})=0
$$

\subsubsection{Conservation of momentum}

The conservation of momentum equation is derived from Newton's second law, which relates the applied forces to the resulting acceleration of a fluid particle with mass. For Newtonian viscous fluids, Navier and Stokes fundamentally derived the following equation using indicial notation (using Stokes hypothesis $\lambda=-\frac{2}{3}$ ) [41]:

$$
\rho \frac{D \mathbf{U}}{D t}=\rho \mathbf{g}-\nabla P+\frac{\partial}{\partial x_{j}}\left[\mu\left(\frac{\partial u_{i}}{\partial x_{j}}+\frac{\partial u_{j}}{\partial x_{i}}\right)+\delta_{i j} \lambda d i v(\mathbf{U})\right]
$$

where $\mathbf{g}$ is the gravitational acceleration vector, $P$ is the total pressure, $x$ is the spatial coordinate, $\mu$ is the dynamic viscosity, $u$ is the velocity component, $\delta_{i j}$ is the Kronecker delta function and $\lambda$ is coefficient of bulk viscosity associated with volume expansion. For incompressible flow, the coefficient of bulk viscosity $\lambda$ and $\operatorname{div}(\mathbf{U})$ (due to the continuity relationship, Equation 3.2) vanish and Equation 3.3 simplifies to:

$$
\rho \frac{D \mathbf{U}}{D t}=\rho \mathbf{g}-\nabla P+\mu \nabla^{2} \mathbf{U}
$$

where the laplacian $\nabla^{2}=\partial / \partial x+\partial / \partial y+\partial / \partial z$.

As mentioned in Section 1.1, for free or "natural" convection, the fluid motion is not generated by an external source (such of a pump or fan, etc.), but rather by the temperature gradients of the fluid. The temperature gradients cause the density of the fluid to change and this change in density is the driving force of the fluid momentum. The total fluid density can be written as a constant or reference density plus some 
change in density:

$$
\rho=\rho_{o}+\rho^{\prime}
$$

where the change in density can be approximated as a function of temperature [42]:

$$
\rho^{\prime} \approx-\rho_{o} \beta\left(T-T_{\infty}\right)
$$

where $\beta$ is the thermal expansion coefficient, $T$ is the temperature of the fluid and $T_{\infty}$ is the reference or inlet temperature. Substituting Equation 3.6 into Equation 3.5 yields the density of the fluid as a function of constant density and temperature:

$$
\rho \approx \rho_{o}\left[1-\beta\left(T-T_{\infty}\right)\right]
$$

Substituting Equation 3.7 into Equation 3.4, and taking into account that $P_{t}=$ $P_{s}+P_{d}+P_{r e f}$ and $\nabla P_{s}=\rho_{0} \mathbf{g}$, gives:

$$
\rho_{o} \frac{D \mathbf{U}}{D t}=-\nabla P_{d}+\mu \nabla^{2} \mathbf{U}-\rho_{o} \mathbf{g} \beta\left(T-T_{\infty}\right)
$$

where $P_{t}$ is the total pressure, $P_{s}$ is the static pressure, $P_{d}$ is the dynamic pressure and $P_{\text {ref }}$ is the reference pressure. Equation 3.8 is know as the Oberbeck-Boussinesq approximation and is valid for an almost incompressible fluid where the temperature changes are assumed sufficiently small relative to the absolute temperature so that the linear density approximation is valid, cf. Equation 3.7.

\subsubsection{Conservation of energy}

The conservation of equation equation is derived from the first law of thermodynamics, which states that the sum of work and heat added to a system will result in an increase in the energy of that system. Neglecting radiation effects, the energy equation in its standard form can be written as [41]:

$$
\rho \frac{D H}{D t}=\frac{D P}{D t}+\operatorname{div}(k \nabla T)+\Phi
$$


where $H$ is the enthalpy, $k$ is the thermal conductivity, $T$ is the temperature of the fluid and $\Phi$ represents the dissipation function, involving viscous stresses, expressed as:

$$
\begin{aligned}
\Phi & =\mu\left[2\left(\frac{\partial u}{\partial x}\right)^{2}+2\left(\frac{\partial v}{\partial y}\right)^{2}+2\left(\frac{\partial w}{\partial z}\right)^{2}\right] \\
& +\mu\left[\left(\frac{\partial v}{\partial x}+\frac{\partial u}{\partial y}\right)^{2}+\left(\frac{\partial w}{\partial y}+\frac{\partial v}{\partial z}\right)^{2}+\left(\frac{\partial u}{\partial z}+\frac{\partial w}{\partial x}\right)^{2}\right] \\
& +\lambda\left(\frac{\partial u}{\partial x}+\frac{\partial v}{\partial y}+\frac{\partial w}{\partial z}\right)^{2}
\end{aligned}
$$

For the limit of low velocity or incompressible flow with constant thermal conductivity, the viscous dissipation becomes negligible and Equation 3.9 simplifies to:

$$
\frac{D T}{D t}=+\alpha \nabla^{2} T
$$

where $\alpha=k / \rho C_{p}$ is the thermal diffusivity and $C_{p}$ is the specific heat capacity.

\subsection{Numerical solution schemes}

The solution of the mass, momentum and energy conservation equations is achieved through the use of numerical approximation schemes; such as the finite difference, finite volume and finite element discretization methods. The purpose of any discretisation method is to transform one or more partial differential equations into a corresponding system of linear algebraic equations. The discretisation process can be split into two components: the discretisation of the computational domain (as discussed in Section 3.3) and the discretisation of the governing equations, discussed below in Section 3.5.1. Section 3.5.2 then gives an overview of how the pressure-velocity coupling is treated in solving the momentum conservation equation.

\subsubsection{Finite volume method}

The discretisation method implemented by OpenFOAM is the Finite Volume Method (FVM), which was originally developed as a special finite difference formulation [36]. 
The Finite Volume Method consists of the following properties [43]:

- This method is based on discretising the integral form of the governing equations over each control volume. The basic quantities, such as the mass and momentum, will therefore be conserved at a discrete level.

- The governing equations are solved at spacial locations, in a fixed Cartesian coordinate system, on the mesh that does not change in time. This method is applicable to both steady-state and transient calculations.

- The control volumes can be of any general polyhedral shape, with a variable number of neighbouring control volumes, thus creating an arbitrarily unstructured or structured mesh. All dependent variables share the same control volumes, which is usually called a collocated or non-staggered variable arrangement [44-46].

- Systems of partial differential equations are treated in a segregated manner [47, 48], meaning that they are solved sequentially one at a time, with the interequation coupling treated in an explicit manner.

The discretisation of the mass, momentum and energy conservation equations, using the Finite Volume Method, has been documented previously in a number of textbooks and papers. The reader is thus invited to go through Chapter 4 of Versteeg [36], Chapter 4 of Ferziger [46] and Chapter 3 of Jasak [43] for more information.

\subsubsection{The PISO algorithm}

In a segregated approach the equations are solved in sequence and special treatment is required in solving the pressure-velocity coupling in the momentum conservation equation. OpenFOAM applies the velocity correction what some would call "in then spirit of Rhie and Chow" [43]. The Rhie-Chow correction is absolutely vital for fluid flow simulations, solved using collocated or non-staggered grids, since it removes oscillations in the solution [49]. The pressure equation is derived from a semi-discretised form of the momentum equation (obtained from the integral form of the momentum equation). This correction is most often seen as the correction of the interpolated cell face velocity, by subtracting the difference between the pressure gradient and the 


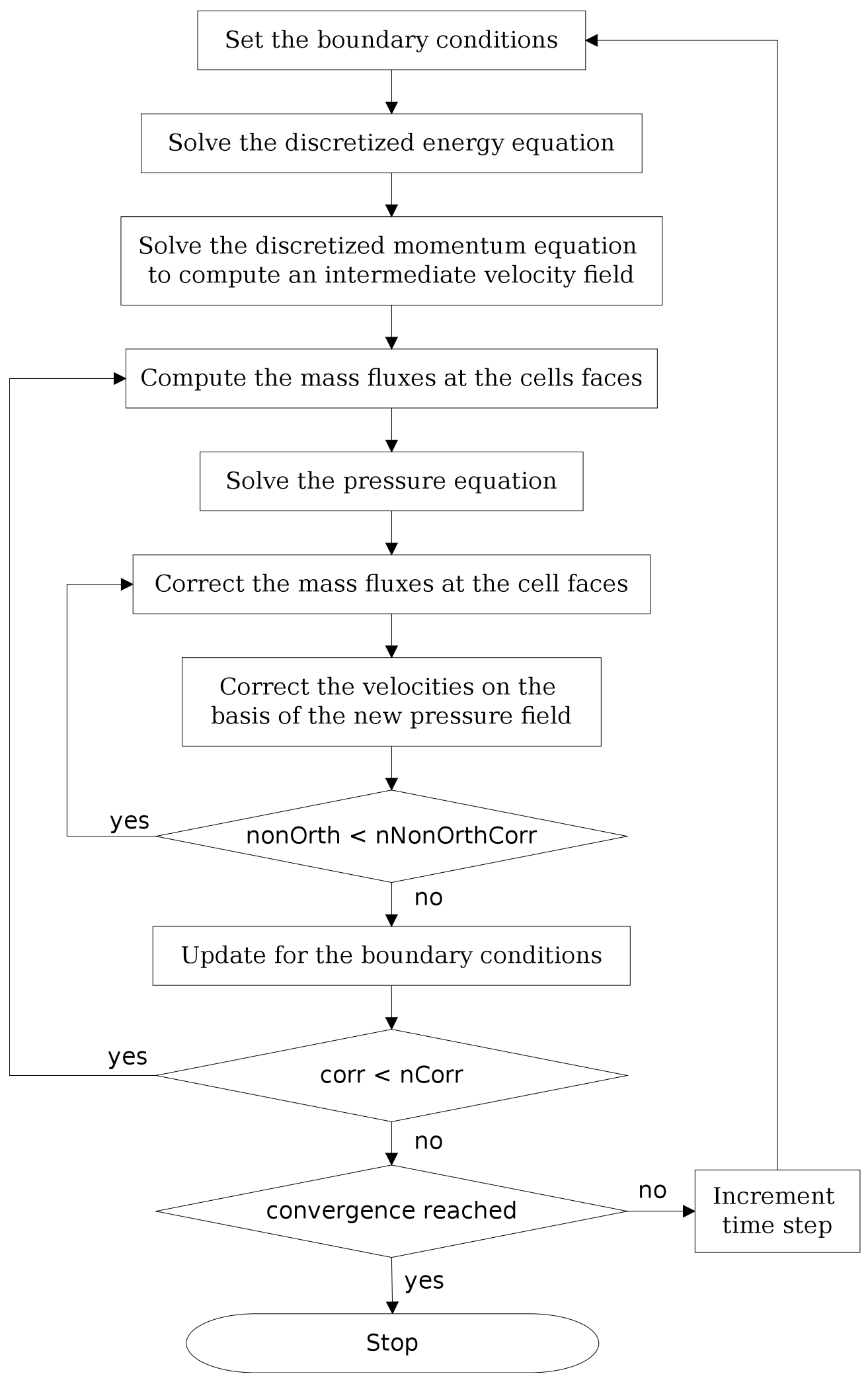

Figure 3.1: The PISO algorithm. 
interpolated gradient at the cell face. This approach to pressure-velocity coupling on collocated grids was developed in the early 1980s and is usually attributed to Rhie and Chow [44]. It is widely used and is employed in many commercial CFD codes [46]. The derivation of the Rhie-Chow interpolation method is shown in Appendix A and the implementation of this method in OpenFoam is shown in Appendix B.

The solution algorithm employing the Pressure-Implicit with Splitting of Operators (PISO) procedure, proposed by Issa [50], can be described as follows [43]:

- The energy equation, Eqn. 3.11, is solved first using the mass fluxes at the cell faces (interpolated from the velocity field) from the previous time-step. This stage is know as the temperature solution.

- The momentum equation is then solved using the new temperature field. The exact pressure gradient source term is not known at this stage and thus the pressure field from the previous time-step is used instead. This stage is called the momentum predictor. The solution of the momentum equation, Eqn. 3.8, gives an approximation of the new velocity field.

- The mass fluxes at the cell faces are then updated based on the intermediate velocity field.

- The semi-discretised form of the velocity equation is then used to formulate the pressure equation. The solution to the pressure equation gives the first estimate to the new pressure field. This step is known as the pressure solution.

- The mass fluxes at the cell faces and the velocity field are corrected based on the new pressure field. This is done for a prescribed number of times (nNonOrthCorr) to account for non-orthogonality in the mesh.

- The procedure of formulating and solving the pressure equation; and correcting the mass fluxes and velocity fields is done for a prescribed number of times (nCorr) before continuing on to the next temperature solution and momentum correction steps.

The flow diagram for this solution algorithm is shown in Fig. 3.1. For further information on how the pressure equation is derived and how the velocity is corrected, the 
reader is invited to go through Chapter 6 of Versteeg and Malalasekera [36], Chapter 7 and 8.8 of Ferziger and Perić [46] and Chapter 3 of Jasak [43].

\subsection{Verification}

The governing equations (Equation 3.1, 3.8 and 3.11) as well as the PISO algorithm were implemented in OpenFOAM as a stand alone "solver". This "solver", shown in Appendix B, was verified for three cases:

- Self driven cavity problem (Section 3.6.1),

- Heated parallel plates (Section 3.6.2) and

- The recreation of the results for stationary cylinders, reported by Bello-Ochende [22], discussed in Sections 6.3.1.1 and 6.3.2.1

\subsubsection{Self driven cavity}

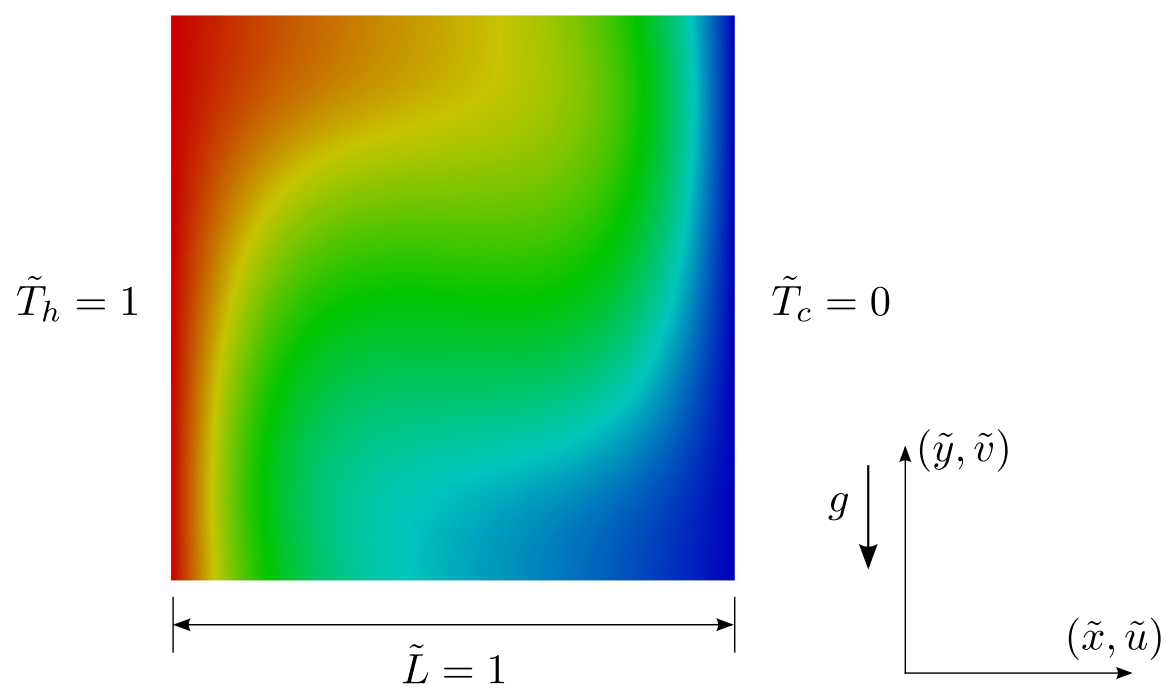

Figure 3.2: Self driven cavity problem.

Consider a two-dimensional square cavity as shown in Fig. 3.2. The velocity boundary condition is specified as zero slip and zero penetration on all sides of the cavity. 
For the left and right sides of the cavity, the surface temperature is assumed constant and uniform, with a high surface temperature on the left side and a low surface temperature on the right side. For the top and bottom sides of the cavity, the temperature boundary conditions is specified as adiabatic. The pressure boundary conditions is specified as zero gradient on all sides of the cavity.

\begin{tabular}{llll}
\hline & \multicolumn{2}{c}{$\tilde{q}$} & \\
\cline { 2 - 3 }$R a$ & Present & Ref. [51] & Difference (\%) \\
\hline $10^{3}$ & 1.116 & 1.118 & 0.179 \\
$10^{4}$ & 2.243 & 2.245 & 0.076 \\
$10^{5}$ & 4.517 & 4.521 & 0.100 \\
$10^{6}$ & 8.731 & 8.822 & 1.027 \\
\hline
\end{tabular}

Table 3.1: Heat transfer rate, per unit length, from the heated side of the cavity to the cooled side of the cavity for $\operatorname{Pr}=0.72$

This problem was simulated for various flow regimes $\left(\operatorname{Pr}=0.72\right.$ and $10^{3} \leq R a \leq$ $10^{6}$ ). From Table 3.1, it can be seen that there is a maximum difference of about $1 \%$ between the results obtained from the present simulations and the results reported by da Silva and Gosselin [51].

\subsubsection{Parallel plates}

Consider the two-dimensional computational domain with two heated parallel plates as shown in Fig. 3.3. For the heated plates, the velocity boundary condition in specified as zero slip and zero penetration; and the surface temperature is assumed constant, uniform and higher than that of the inlet stream. For the inlet of the computational domain, the boundary conditions are specified as $\tilde{P}=0, \tilde{T}_{\infty}=0$ and $\tilde{u}=\partial \tilde{v} / \partial \tilde{y}=0$. For the exit of the computational domain, the boundary conditions are specified as $\partial(\tilde{u}, \tilde{v}) / \partial \tilde{y}=0, \partial \tilde{P} / \partial \tilde{y}=0$ and $\partial \tilde{T} / \partial \tilde{y}=0$.

This problem was simulated for a fixed flow regime $\left(\operatorname{Pr}=0.72\right.$ and $\left.R a=10^{6}\right)$ and the plate-to-plate spacing was varied. There exists an optimum plate-to-plate spacing, shown in Fig. 3.4, of $\tilde{S}_{o,{ }_{o p t}} R a^{1 / 4}=2.790$ or $\tilde{S}_{o,{ }_{\text {opt }}}=0.088$. There is less than a $1.15 \%$ difference when comparing this optimal spacing with that reported by da Silva and Bejan [20]. 


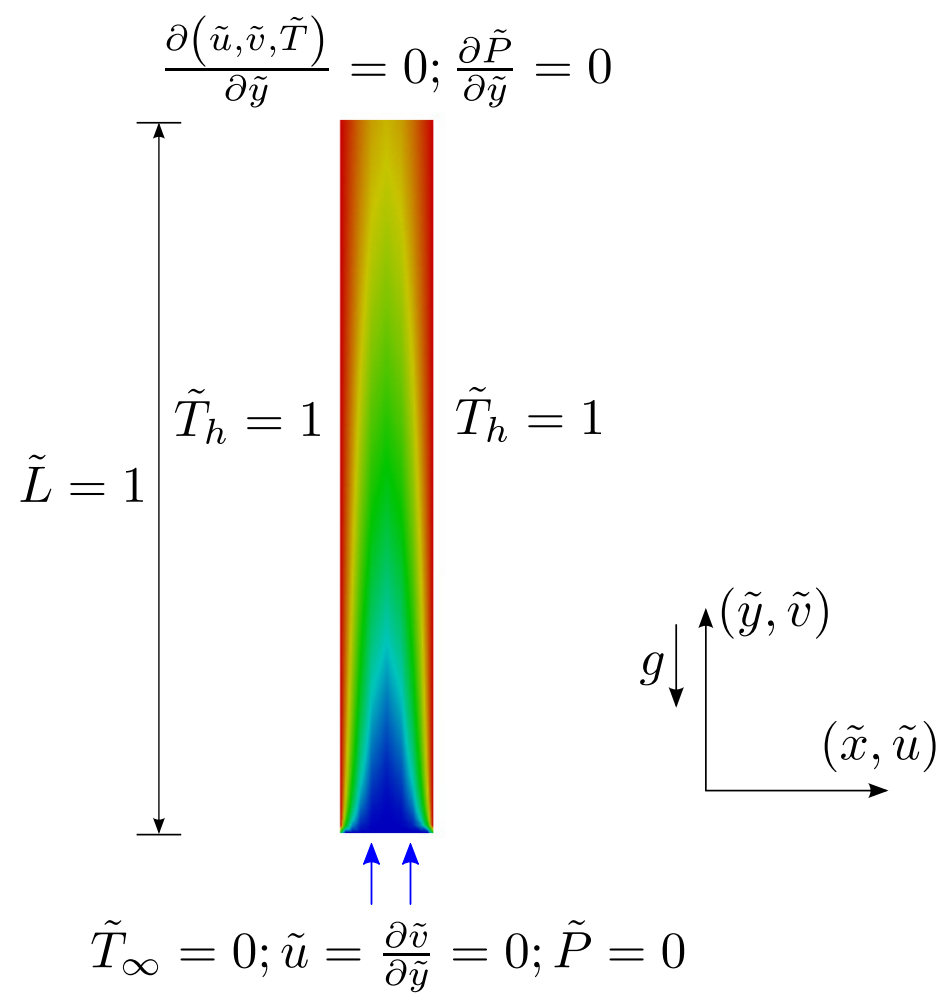

Figure 3.3: Heated parallel plates cooled by natural convection.

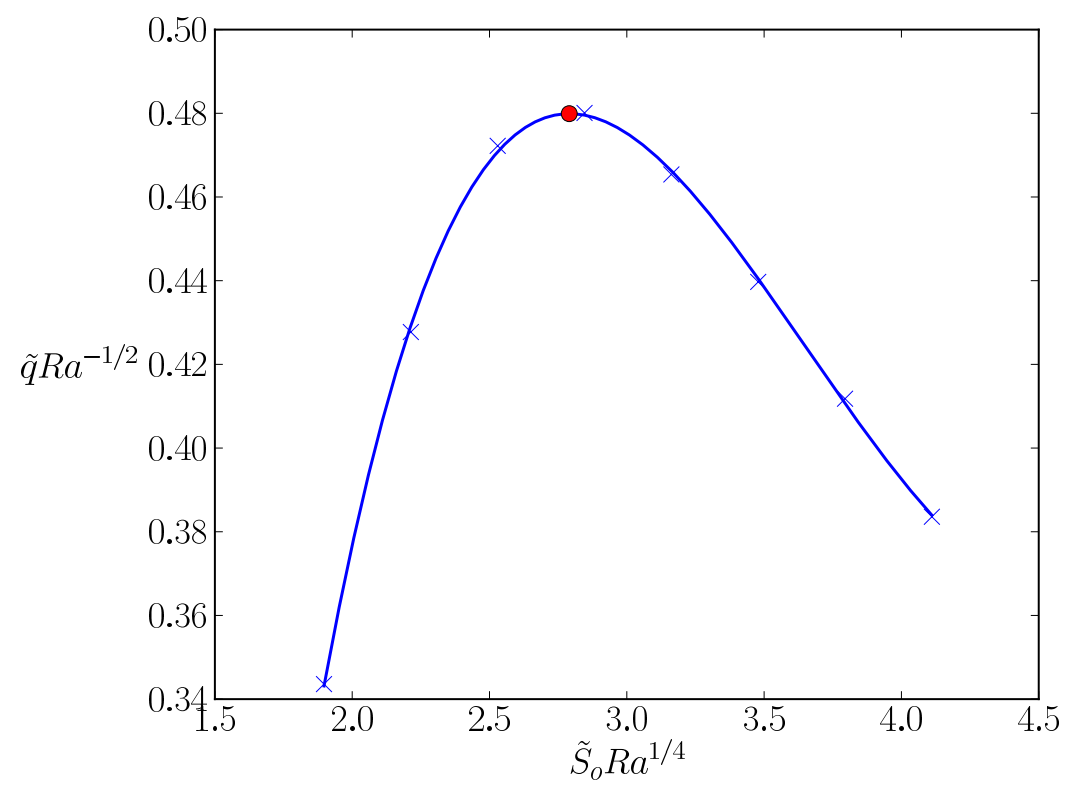

Figure 3.4: The effect of the plate-to-plate spacing on the heat transfer density rate for $\operatorname{Pr}=0.72$ 
The corresponding maximum heat transfer density rate is $\tilde{q}_{m} R a^{-1 / 2}=0.480$ or $\tilde{q}_{m}=478$. There is less than a $8.35 \%$ difference when comparing this maximum heat transfer density rate with that reported by da Silva and Bejan [20].

\subsection{Conclusion}

This chapter presented an overview of the processes involved in setting up and solving a typical engineering heat transfer and fluid flow problem through the use of a CFD package or code. The set of non-linear partial differential equations that govern conservation of mass, momentum and energy where discussed. The numerical methods and algorithms employed by OpenFOAM in solving the governing equations was also reviewed. Finally the implementation of this these numerical methods and algorithms in OpenFOAM were verified for two example cases. 


\section{Chapter 4}

\section{Parametrisation and optimization}

\subsection{Introduction}

Almost all CFD packages or codes are designed with the goal in mind that the CFD package will be used once off for a given engineering problem with maybe a handful of variations in either the geometry or fluid properties being simulated. For an optimisation type problem this "one-by-one set up and solution" method can become very cumbersome as the number of simulations required to be set up and solved becomes exponentially greater with an increased number of design variables. A more efficient method is sought and this chapter deals with just that. In order to automate this entire process three key aspects to interface with OpenFoam are highlighted:

1. Initialisation: In which all parameters need to be set (fluid properties, gravitational acceleration, fixed boundary conditions etc.). All variables also need to be set and the discretisation of the computational domain (mesh generation) is also done at this stage.

2. Execution: Here a system call to execute OpenFoam's solver is required. Some form of monitoring of the solution residuals is also required so that a system termination call can be issued to stop OpenFoam.

3. Analysis: In which the results from the simulation are extracted and consolidated into a single log file for ease of review and comparison. 
Section 4.2 gives an introduction to the tools used to achieve this automation process. Section 4.3 shows how these tools are coupled together with OpenFoam for an automated parametrisation set up. Section 4.4 discusses the principals behind optimisation and the governing equations for the optimisation algorithm used as well as how this optimisation algorithm is easily merged with the parametrisation process routine.

\subsection{Open source tools}

The tools used in this thesis to interface with OpenFoam and to automate the simulation process are all open source and freely available:

- Python: An open source cross platform programming language [40].

- Gnuplot: A portable command-line driven graphing utility for multiple platforms [52].

- NumPy: A Python library, adding support for multi-dimensional arrays and matrices, along with a large library of high-level mathematical functions to operate on these arrays [53].

- SciPy: An Open Source library of scientific tools for Python. It depends on the NumPy library, and it gathers a variety of high level science and engineering modules together as a single package [53].

- PyFoam: A Python library to control OpenFoam simulations and manipulate OpenFoam data [54].

Python is used as the "glue" that couples all the tools discussed above together in order to create a seamlessly smooth automated script. Python is also the key tool used to create the geometry and mesh input file, as discussed in section 3.3. Python was chosen as the programming language for several reasons: python is very easy to learn, the code is extremely readable, the code is dynamic and there are numerous comprehensive libraries available for scientific and mathematical functions. Gnuplot is used to actively plot the solution residuals during or after the simulation. SciPy is used for its readily availably, built in optimisation algorithms. PyFoam is the key tool used 
to interface with OpenFoam as it is able to manager and control the "Initialisation" and "Execution" aspects of the OpenFoam interfacing.

\subsection{Parametrisation}

Parametrisation is the method whereby the simulation case files (geometry and mesh input, fluid properties, boundary conditions, etc.) are set up in the form of variables or parameters to describe a given system. The two areas of the numerical model that were selected for parametrisation were the geometry and mesh input file and the velocity boundary condition file (effecting the cylinder rotation speed). The geometry file was automated following the method described in section 3.3 through the use of Python. This script was set up with variable and parameter information (discussed later in section 5.2) being parsed to the python script and the required OpenFoam input file being automatically generated. The complete parametrisation flow diagram in shown in Fig. 4.1. From this flow diagram is can be seen that the entire process from mesh generation to extracting the results from the OpenFoam simulation has been automated through the use of python and the set of python tools (discussed in section 4.2); and this process loops over a given range for a given variable or parameter. The python scripts have been attached digitally in the data attachment in Appendix C.

\subsection{Numerical optimisation}

This section presents the theory behind mathematical optimisation and introduces the optimisation algorithm used in this thesis. The feasibility of using an optimisation algorithm becomes prevalent for design cases with two or more design variables as it eliminates the need to consider each and every result generated by range of design variables combinations, but rather seeks out the optimal results from the information obtained from a fewer number of design variable combinations. 


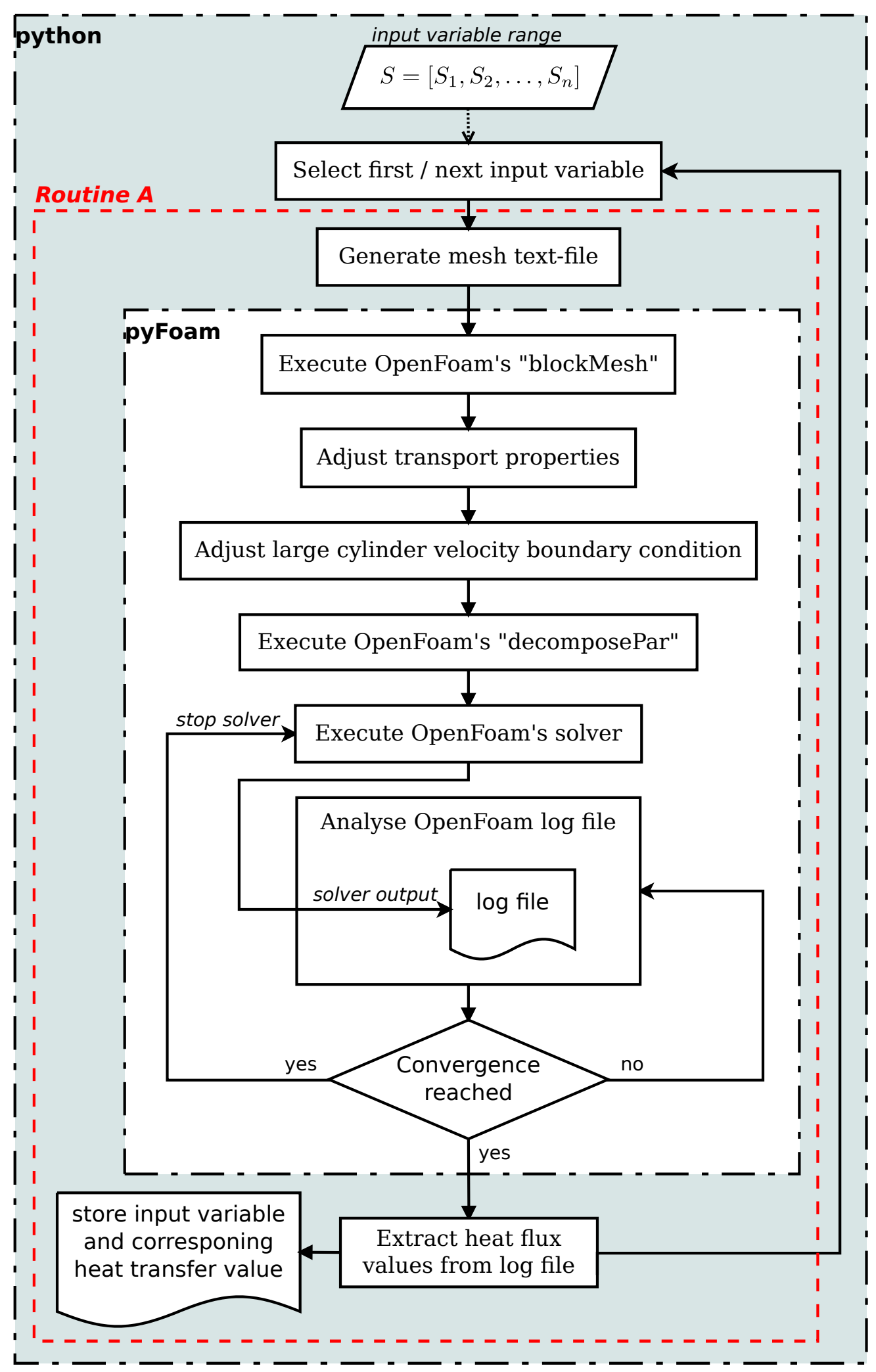

Figure 4.1: Parametrisation routine for OpenFoam 


\subsubsection{Numerical optimisation overview}

Numerical optimisation (also known as mathematical optimisation, mathematical programming or non-linear programming) is the field that deals with determining the best solution to problems which can be expressed mathematically or numerically. In other words, it implies choosing the best result from a range of available alternatives for a given range of variables. The history of this field dates back to the 1940s when the first optimisation technique, called the steepest descent, was developed for solving very simple problems. Since then numerous methods for solving a general optimisation problem have been developed, tested and successfully applied to many important scientific, economic and engineering problems [55]. One such successful application of an automated mathematical optimisation algorithm coupled to a commercial CFD package is presented by Bello-Ochende et al. [56] and Ighalo [57], where the authors utilised the Dynamic-Q algorithm developed by Snyman and Hay [58].

\subsubsection{Non-linear constrained optimisation}

In numerical optimisation, the quantity to be optimised (minimised or maximised) is known as the objective or cost function $f(\mathbf{x})$. The parameters or variables to be changed in order to obtain this optimal solution are known as the design variables and they are usually represented by a vector $\mathbf{x}$ with the optimal values usually expressed by the vector $\mathbf{x}^{*}$. When certain constraints in the form of inequalities $g_{i}(\mathbf{x}) \leq 0$ or equalities $h_{j}(\mathbf{x})=0$ are introduced into the process, the problem or objective function is known as a constrained optimisation problem otherwise the problem is known as an unconstrained optimisation problem. In general, a constrained optimisation problem is formally written as:

$$
\begin{array}{ll}
\underset{w . r . t . \mathbf{x}}{\operatorname{minimise}} & \\
& f(\mathbf{x}), \quad \mathbf{x}=\left[x_{1}, x_{2}, \ldots, x_{n}\right]^{T} \in \mathbb{R}^{n} \\
\text { such that: } & \\
& g_{i}(\mathbf{x}) \leq 0, \quad i=1,2, \ldots, m \\
& h_{j}(\mathbf{x})=0, \quad j=1,2, \ldots, r
\end{array}
$$




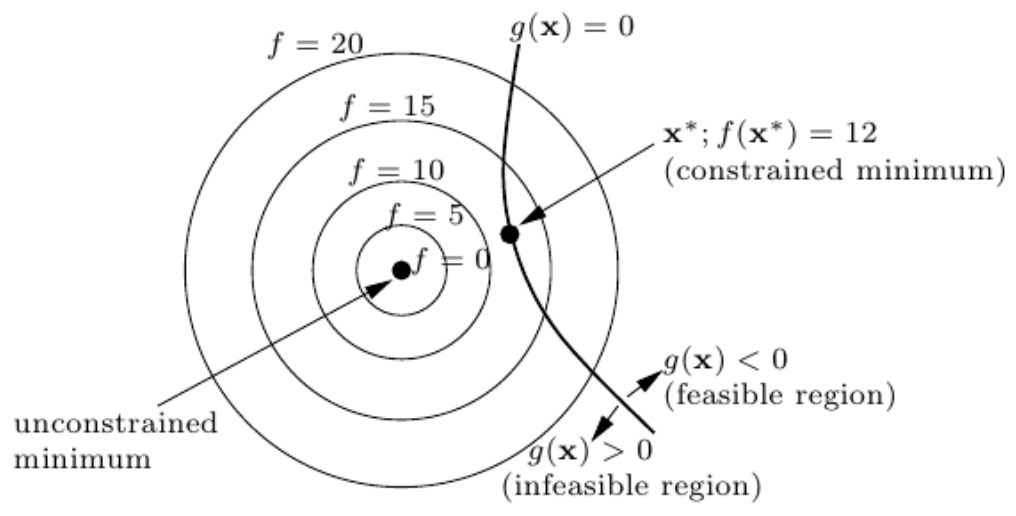

(a) Inequality Constraint

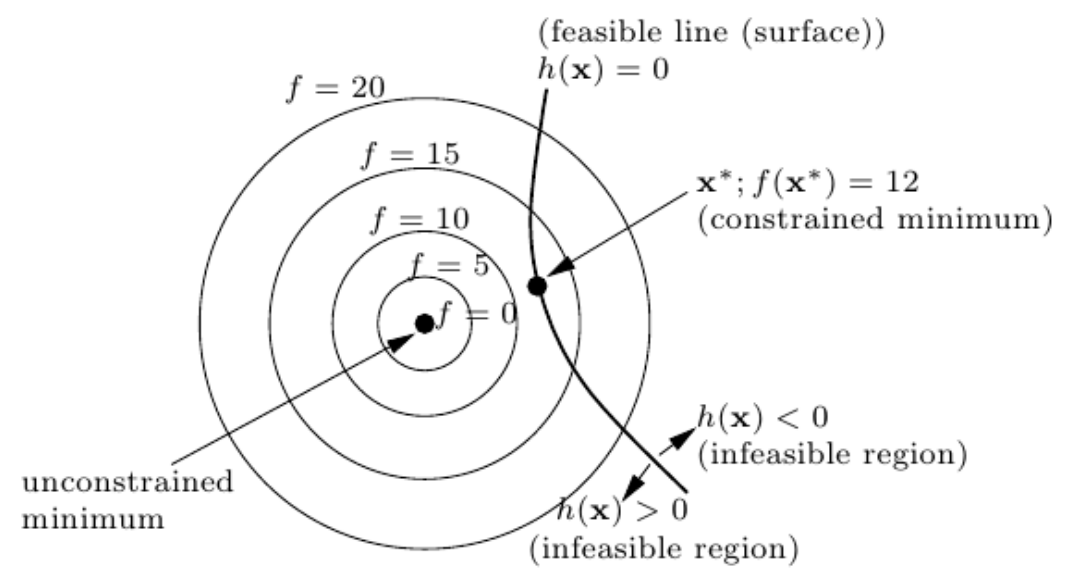

(b) Equality Constraint

Figure 4.2: Contour representation of a constrained problem [55]

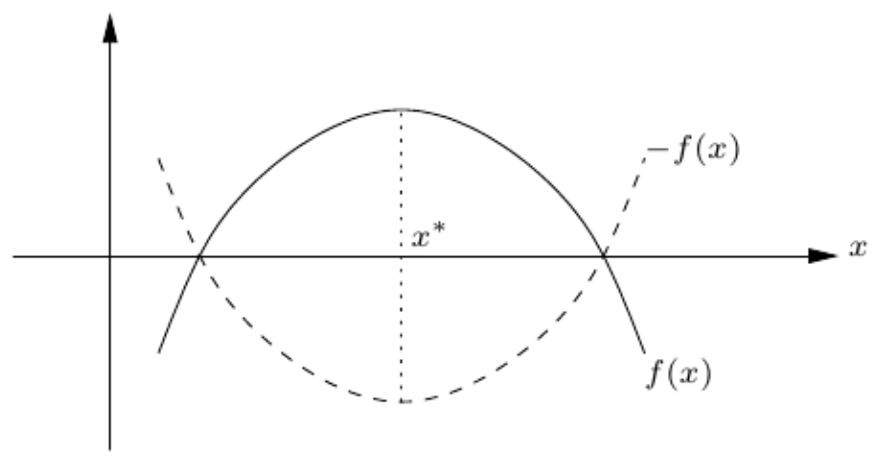

Figure 4.3: Illustration of a maximisation function versus a minimisation function [55]. 
Figure 4.2 shows the difference between an inequality constraint and an equality constraint and how these constraints effect the feasible region (the region in which the solution is valid and does not violate any of the constraints). In the case where the objective function $f(\mathbf{x})$ is required to be maximised, the minimisation algorithm is still applied by setting $f_{\min }(\mathbf{x})=-f_{\max }(\mathbf{x})$. The plot in Fig. 4.3 depicts how the maximisation problem is transformed into a minimisation problem [55].

\subsubsection{Numerical optimisation algorithm}

For most optimisation algorithms it is required that the objective or cost function be known, smooth and continuous with one optimum solution (being the global optimum). Difficulties arise when these conditions are not met, for example when oscillations occur in the objective function it becomes difficult to evaluate the oscillations as only local minimums and still return a global minimum. If discontinuities occur in the objective function no function or gradient information is available at the point or range of the discontinuity making it difficult to evaluate a global minimum.

When there is no analytical expression for the objective function (as is the case in many heat transfer and fluid dynamics problems), the gradient information of the objective function is not readily available and approximation methods are required in order to obtain this gradient information. These gradient approximation methods often require additional objective function evaluations, making them very "expensive" based on the time required for each objective function evaluation. Each objective function evaluation is the solution of a CFD simulation. Many optimisation algorithms can be found that have been developed to deal with the difficulties mentioned above, some such algorithms are presented in Ref [58-63]

Unfortunately there are a limited number of optimisation algorithms available in the SciPy python library when considering multiple design variables and constraint functions. The objective function is thus minimised using the Sequential Least SQuares Programming (SLSQP) algorithm available in the SciPy python library "fmin_slsqp" [53]. This algorithm is based on the Fortran routine originally implemented by Kraft [64] which utilises the slightly modified Non-Negative Least Squares (NNLS) algorithm of Lawson [65]. The detail of optimisation algorithm itself is outside the scope of this thesis, thus the reader is invited to go through Ref $[64,65]$ for more information. 


\subsubsection{Automation}

The optimisation algorithm presented in section 4.4.3 is easily merged with the automated parametrisation subroutine as shown in the process flow diagram in Fig. 4.4. "Routine A" from the parametrisation process shown in Fig. 4.1 effectively becomes the objective function $f(\mathbf{x})$. For a given design variable vector $\mathbf{x}^{i}$, where $\mathbf{x}^{0}$ (the initial or starting values for the design variables) has to be provided by the user, the optimisation algorithm calculates the values of the cost function $f\left(\mathbf{x}^{i}\right)$; its gradient $\nabla f\left(\mathbf{x}^{i}\right)$; the constraints $g\left(\mathbf{x}^{i}\right), h\left(\mathbf{x}^{i}\right)$, and their Jacobi matrices $\nabla g\left(\mathbf{x}^{i}\right), \nabla h\left(\mathbf{x}^{i}\right)$. These values are introduced into the optimiser which either proposes a new design variable vector $\mathbf{x}^{i+1}$ or informs the user that certain convergence criteria have been satisfied and terminates [64].

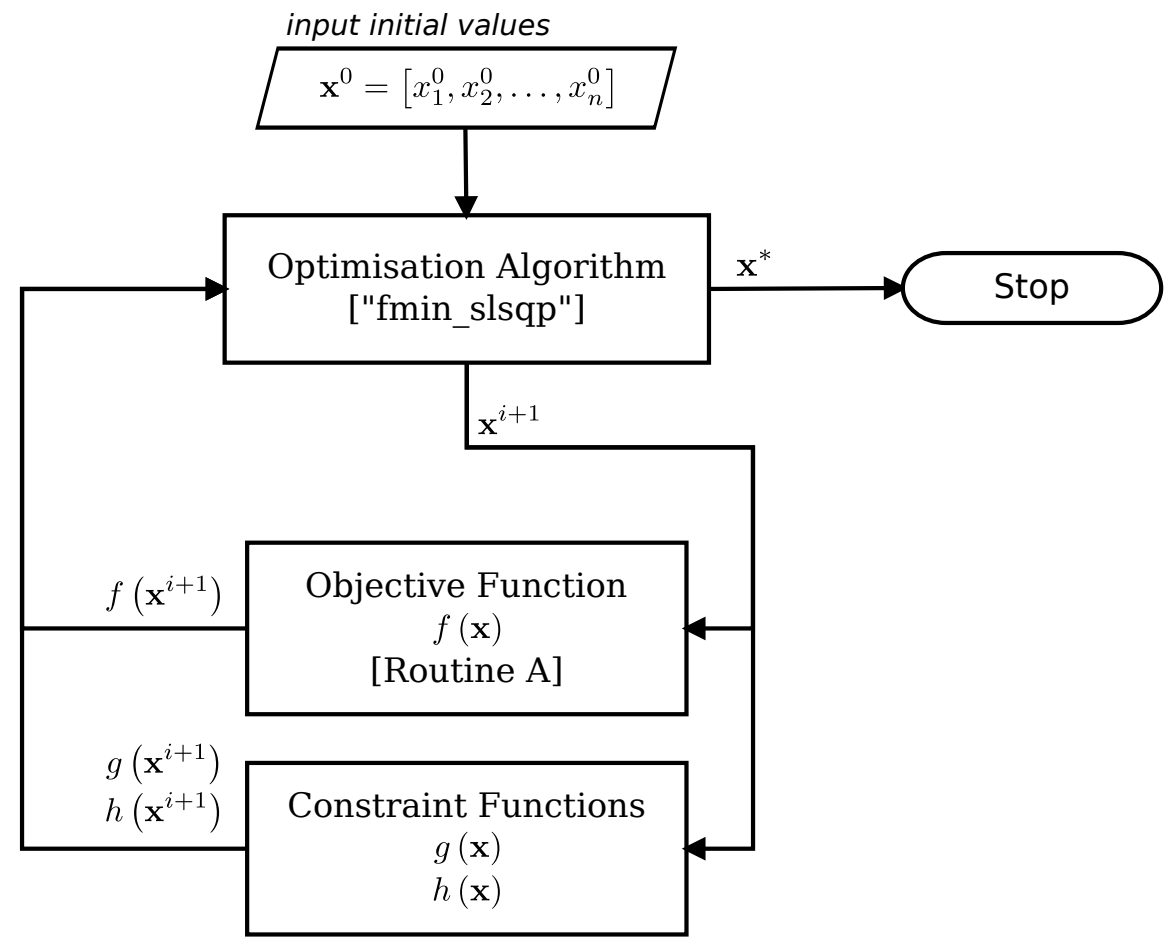

Figure 4.4: Optimisation routine for OpenFoam.

\subsection{Conclusion}

This Chapter presented the tools used to automate the numerical computational process and how these tools where implemented to create a parameterisation routine to 
interface with OpenFoam. This Chapter also presents an efficient and effective method for solving geometric optimisation design type problems whereby a mathematical optimisation algorithm is coupled to a Computation Fluid Dynamics (CFD) package through the use of a programming language in order to efficiently optimise the design problem (with multiple design variables) for a global objective subject to global constraints. 


\section{Chapter 5}

\section{Optimization of rotating cylinders in natural convection}

\subsection{Introduction}

This chapter starts with introducing the numerical model used in OpenFoam to solve the fluid flow and heat transfer fields for heat generating cylinders rotating in natural convection (Section 5.2). Section 5.3 shows how the governing equations expressing the conservation of mass, momentum and energy are non-dimensionalised for the discussed numerical models. Section 5.4 then discusses the objective function used to evaluate the geometric configurations in order to determine the optimum. Section 5.5 presents the convergence criteria used in the OpenFoam solution scheme (Figure 3.1) as well as the convergence criteria used for the optimisation algorithm. The results from a series of studies conducted in order to achieve results independent of computational domain sizes and mesh size is also presented in section 5.5.

\subsection{The numerical CFD model}

This thesis builds on the research previously conducted by Bello-Ochende and Bejan [22] (in which the authors present the optimal geometric configuration and thus the 
corresponding maximum heat transfer density rate for stationary cylinders cooled by natural convection) by considering the effect of rotation on the cylinders. This Section is presented in two smaller sub-sections: the numerical model for a simple single scale geometry structure (Section 5.2.1) and the numerical model for an increase in complexity of the geometric structure or multi scale geometry structure (Section 5.2.2).

\subsubsection{Single scale}

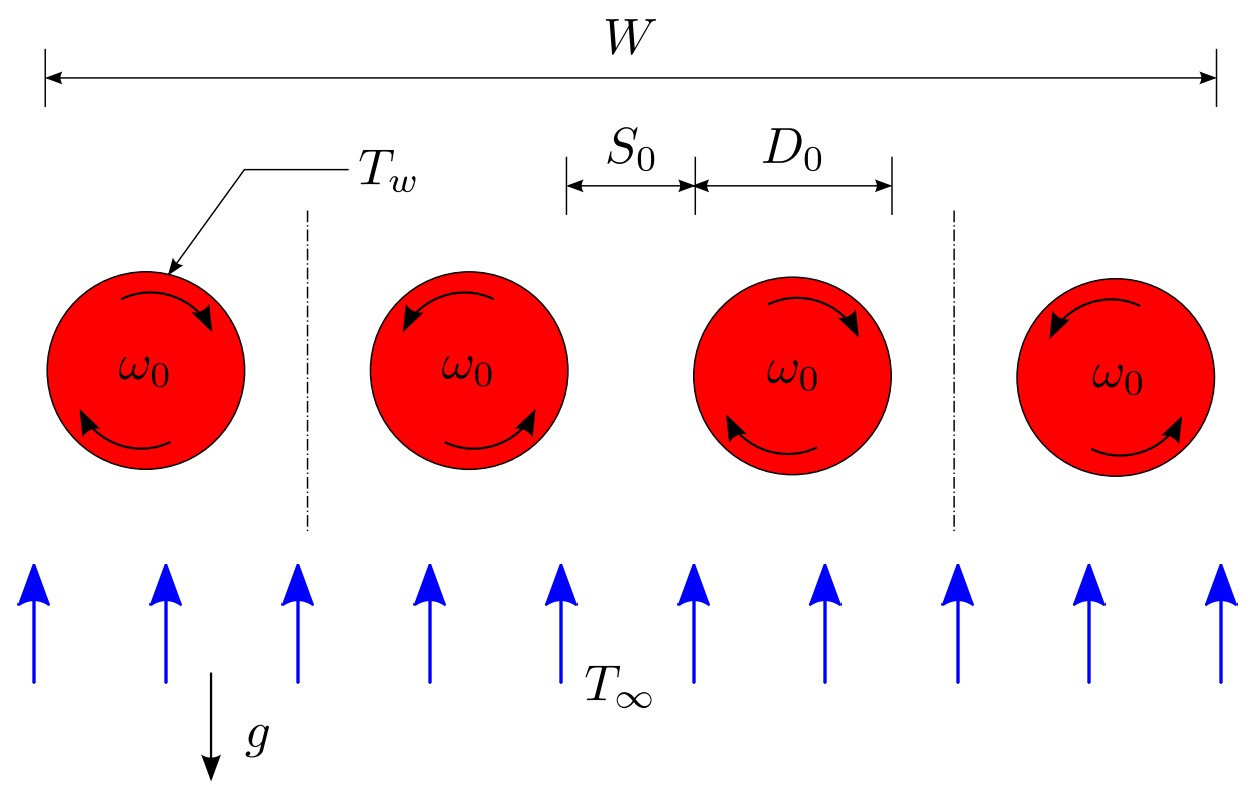

Figure 5.1: Single row of counter-rotating cylinders in natural convection.

Consider a row of infinity long, rotating and heat-generating parallel cylinders aligned along a single line to form a stacking as shown in Fig. 5.1. The cylinders rotate at an angular velocity of $\omega_{0}$ in a counter-rotating configuration. The large cylinder diameter $\left(D_{0}\right)$ is fixed and the surface temperature of the cylinders $\left(T_{w}\right)$ is assumed uniform and constant and greater than that of the fluid temperature $\left(T_{\infty}\right)$. The cylinders thus generate heat and are subsequently cooled by natural convection.

The objective is to select the number of cylinders in the stacking or the cylinder-tocylinder spacing $\left(S_{0}\right)$ in such a manner that the overall thermal heat transfer between the cylinders and the ambient air is maximized. This is done for each flow regime (Rayleigh number) and cylinder rotation speed. The flow is assumed steady, laminar, incompressible and two-dimensional. All thermophysical properties are assumed 
constant. The temperature variations are assumed sufficiently small relative to the absolute temperature so that the Boussinesq approximation is valid.

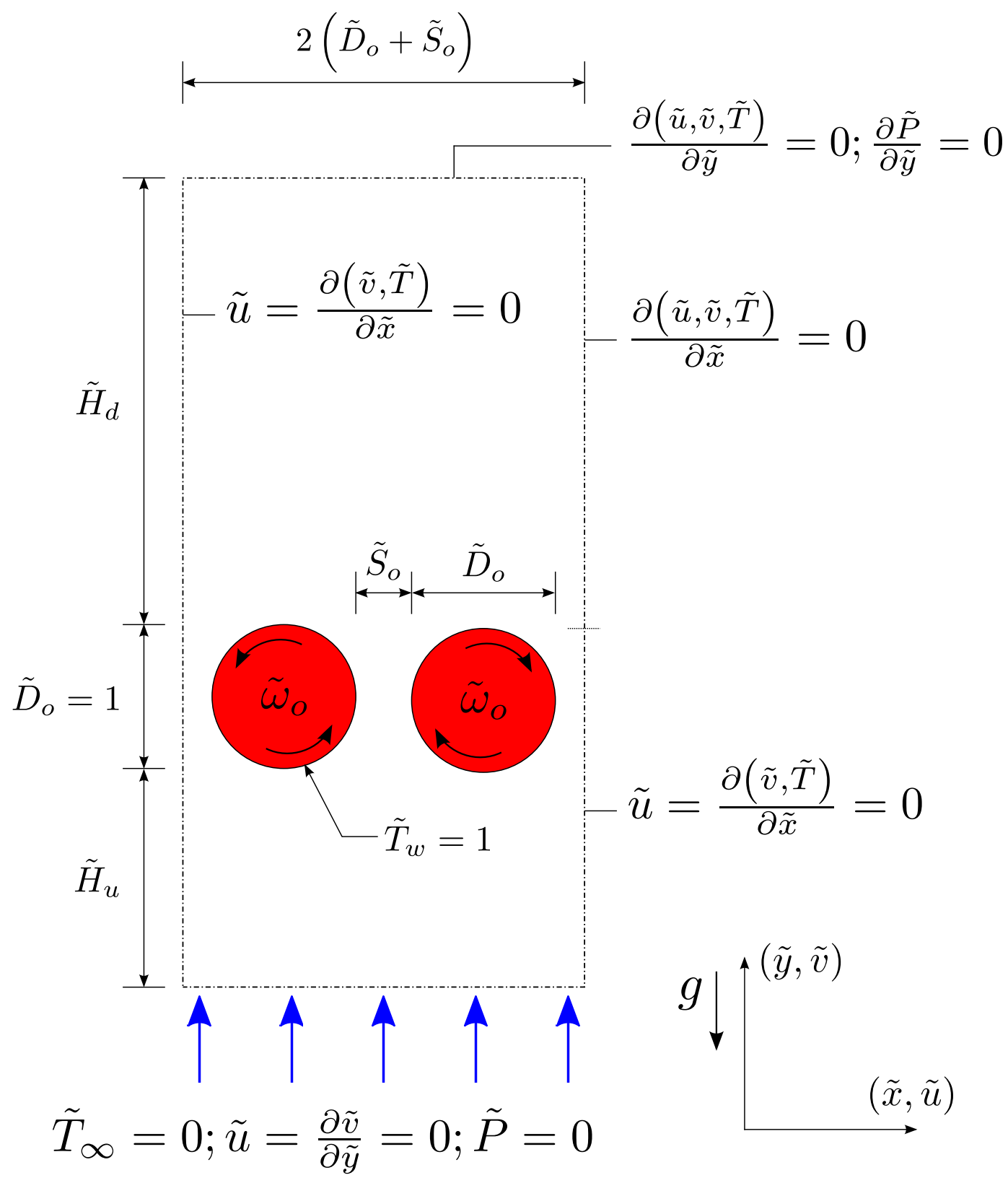

Figure 5.2: The computational domain and boundary conditions for a set of counterrotating cylinders.

Figure 5.2 shows the elemental volume that characterises this assembly. The computational domain contains the upstream section $\left[H_{u} \times 2\left(D_{0}+S_{0}\right)\right]$, the downstream section $\left[H_{d} \times 2\left(D_{0}+S_{0}\right)\right]$ and the flow region $\left[D_{0} \times 2\left(D_{0}+S_{0}\right)\right]$. The upstream lengths $\left(H_{u}\right)$ and downstream lengths $\left(H_{u}\right)$ were selected based on mesh independence tests 
described later in Section 5.5. The computational domain boundary conditions are indicated in Fig. 5.2. For the cylinder surfaces, the boundary conditions are specified as zero slip, zero penetration, constant uniform surface temperature $\tilde{T}_{w}=1$ and an angular velocity of $\tilde{\omega}_{0}$. For the inlet of the computational domain, the boundary conditions are specified as $\tilde{P}=0, \tilde{T}_{\infty}=0$ and $\tilde{u}=\partial \tilde{v} / \partial \tilde{y}=0$. For the exit of the computational domain, the boundary conditions are specified as $\partial(\tilde{u}, \tilde{v}) / \partial \tilde{y}=0, \partial \tilde{P} / \partial \tilde{y}=0$ and $\partial \tilde{T} / \partial \tilde{y}=0$. From the inlet and outlet boundary conditions is it evident that no $\tilde{v}$ velocity component is specified and only a zero gradient boundary condition is specified. The $\tilde{v}$ velocity component is thus generated, in the computational domain, from the buoyancy body force acting on the fluid (cf. Eq. 1.3 and Eq. 3.8).

For the upstream section $\left(0 \leq \tilde{y} \leq \tilde{H}_{u}\right)$ of the computational domain, the boundary conditions are specified as symmetry planes or free slip and no penetration $(\tilde{u}=$ $\partial(\tilde{v}, \tilde{T}) / \partial \tilde{x}=0)$. For the downstream section $\left(\tilde{H}_{u} \leq \tilde{y} \leq \tilde{H}_{u}+\tilde{D}_{0}+\tilde{H}_{d}\right)$ of the computational domain, two boundary conditions are specified: symmetry plane or free slip and no penetration $(\tilde{u}=\partial(\tilde{v}, \tilde{T}) / \partial \tilde{x}=0)$ at the left side of the flow region and; zero stress $(\partial \tilde{P} / \partial \tilde{x}$ and $\partial \tilde{u} / \partial \tilde{x}=\partial(\tilde{v}, \tilde{T}) / \partial \tilde{x}=0)$ on the right side of the flow region. By specifying $\partial \tilde{u} / \partial \tilde{x}$ on the right side of the flow region, fluid is allowed to flow horizontally into the computational domain. This entrainment effect nullifies the unrealistic vertical acceleration or chimney effect that would have been generated had we specified zero slip on this side. The vertical acceleration is also generated should a periodic boundary condition be applied on the left and right side of the entire computational domain.

\subsubsection{Multi scale}

The effect of increased complexity is shown in Fig. 5.3, in which a smaller cylinder (of diameter $D_{1}$ ) is inserted in the entrance (converging) region of the channels formed between the larger cylinders. This geometric change adds one more degree of freedom to the system: the smaller cylinder diameter $\tilde{D}_{1}=D_{0} / D_{1}$. The flow configuration now has two degrees of freedom, represented by the small cylinder diameter $\tilde{D}_{1}$ and the original cylinder-to-cylinder spacing $\tilde{S}_{0}$. Again the objective is to select the number of cylinders (large and small) in the stacking or in other words to select the cylinder-tocylinder spacing $\left(S_{0}\right)$ and the small cylinder diameter $\left(D_{1}\right)$ in such a manner that the 
overall thermal heat transfer between the cylinders and the ambient air is maximized.

The smaller cylinders have a uniform and constant surface temperature $\left(\tilde{T}_{w}\right)$ equal to the surface temperature of the larger cylinders. The smaller cylinders are kept stationary, thus creating a staggered rotational configuration of counter rotating larger cylinders and stationary smaller cylinders. All the cylinders were aligned along their leading edges. It was assumed in this thesis that this alignment was the most beneficial for enhancing the heat transfer density rate for both stationary and rotating cylinders (see Ref. [35]).

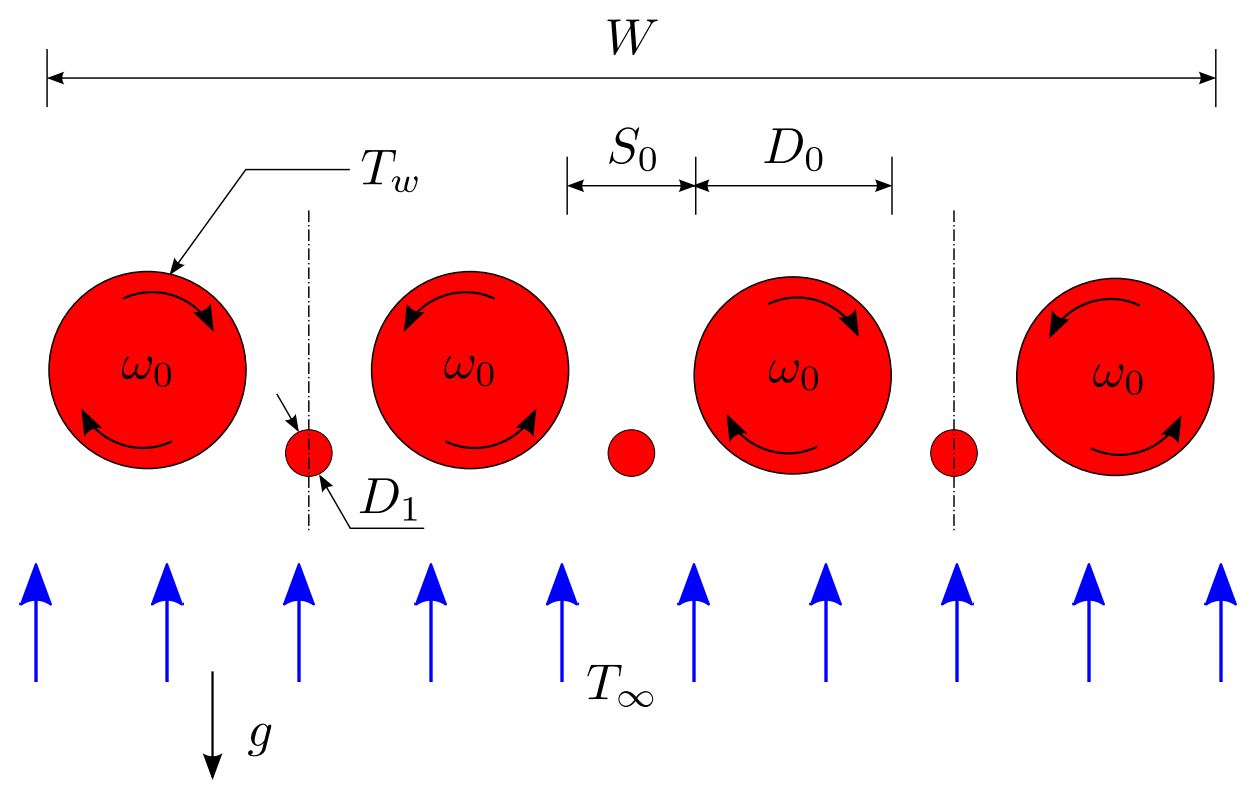

Figure 5.3: Multi scale row of counter-rotating cylinders in natural convection.

Figure 5.4 shows the elemental volume that characterises this assembly. The computational domain boundary conditions, indicated in Fig. 5.4, for the multi scale structure are similar to the single scale structure (Section 5.2.1), with a few differences for the upstream and downstream section boundary conditions. For the upstream section $\left(0 \leq \tilde{y} \leq \tilde{H}_{u}\right)$ of the computational domain, the boundary conditions are specified as symmetry planes or free slip and no penetration $(\tilde{u}=\partial(\tilde{v}, \tilde{T}) / \partial \tilde{x}=0)$. For the downstream section $\left(\tilde{H}_{u} \leq \tilde{y} \leq \tilde{H}_{u}+\tilde{D}_{0}+\tilde{H}_{d}\right)$ of the computational domain, two boundary conditions are specified: symmetry plane or free slip and no penetration $(\tilde{u}=\partial(\tilde{v}, \tilde{T}) / \partial \tilde{x}=0)$ at the left side of the flow region and; zero stress $(\partial \tilde{P} / \partial \tilde{x}$ and $\partial \tilde{u} / \partial \tilde{x}=\partial(\tilde{v}, \tilde{T}) / \partial \tilde{x}=0)$ on the right side of the flow region. 


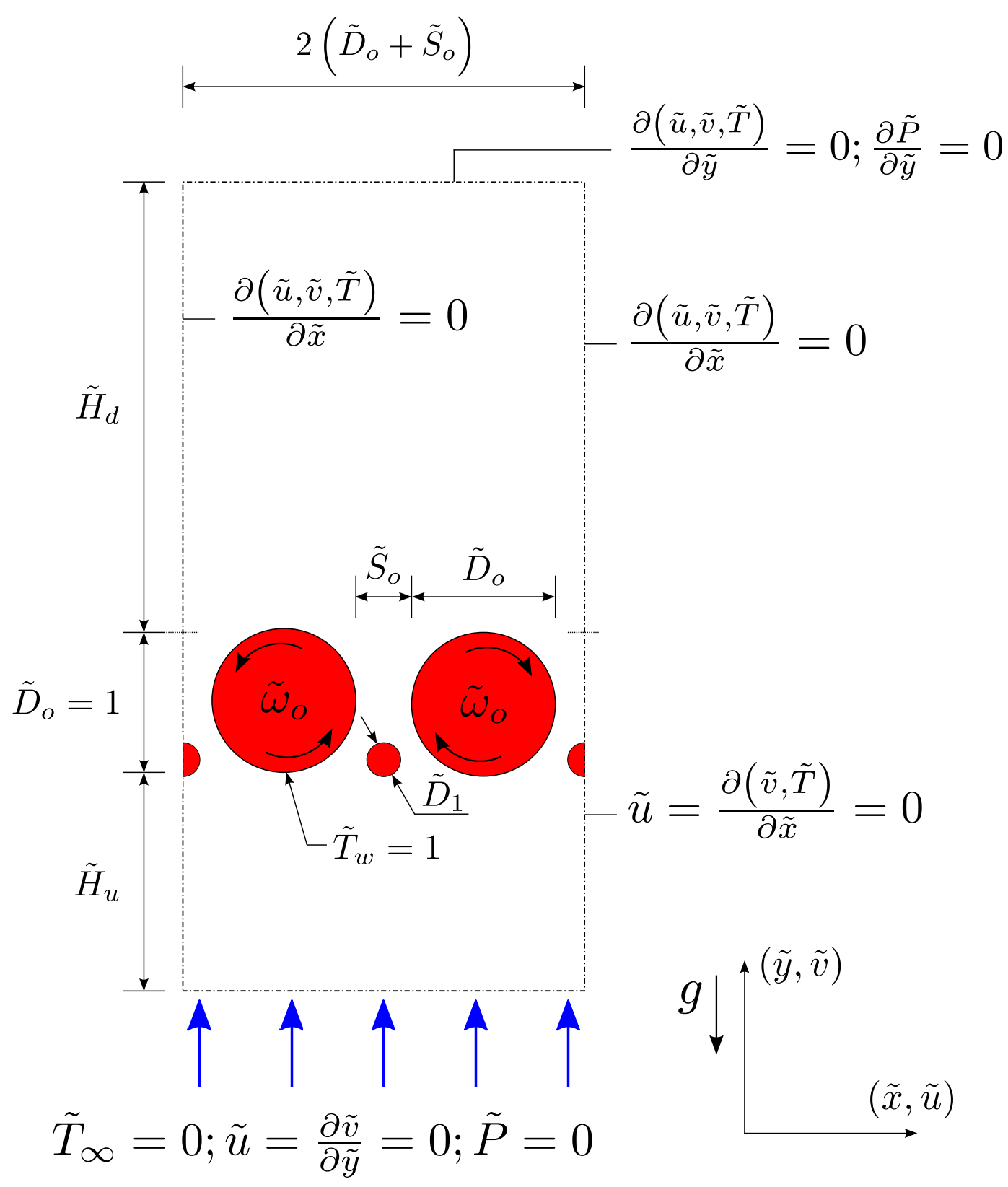

Figure 5.4: The computational domain and boundary conditions for a set of counterrotating cylinders.

\subsection{Non-dimensional governing equations}

The governing continuity of mass, momentum and energy equations (Equations 3.2, 3.8 and 3.11) can be re-written in a non-dimensional form by introducing the following dimensionless variables: 


$$
\begin{gathered}
\tilde{x}, \tilde{y}, \tilde{y}=\frac{(x, y, z)}{D_{0}}, \quad \tilde{u}, \tilde{v}, \tilde{w}=\frac{(u, v, w)}{\left(\alpha / D_{0}\right)\left(R a_{D_{0}} P r\right)^{1 / 2}} \\
\tilde{T}=\frac{T-T_{\infty}}{T_{w}-T_{\infty}}, \quad \tilde{P}=\frac{P}{\left(\alpha \mu / D_{0}^{2}\right)\left(R a_{D_{0}} P r\right)^{1 / 2}} \\
\tilde{\omega}_{0}=\frac{\omega_{0}}{\left(2 \alpha / D_{0}^{2}\right)\left(R a_{D_{0}} P r\right)^{1 / 2}}
\end{gathered}
$$

where the large cylinder diameter $\left(D_{0}\right)$ is selected as the characteristic length, $\operatorname{Pr}=\nu / \alpha$ is the Prandtl number and the Rayleigh number is defined in terms of the large cylinder diameter as:

$$
R a_{D_{0}}=\frac{g \beta\left(T_{w}-T_{\infty}\right) D_{0}^{3}}{\alpha \nu}
$$

Substituting Equations 5.1 to 5.4 into Equations 3.2, 3.8 and 3.11 yields the dimensionless form of the continuity, momentum and energy equations respectively:

$$
\begin{gathered}
\operatorname{div} \tilde{\mathbf{U}}=0 \\
\left(\frac{R a}{P r}\right)^{1 / 2} \frac{D \tilde{\mathbf{U}}}{D \tilde{t}}=-\tilde{\nabla} \tilde{P}+\tilde{\nabla}^{2} \tilde{\mathbf{U}}+\left(\frac{R a}{P r}\right)^{1 / 2} \tilde{T} \hat{k} \\
(\operatorname{RaPr})^{1 / 2} \frac{D \tilde{T}}{D \tilde{t}}=+\tilde{\nabla}^{2} \tilde{T}
\end{gathered}
$$

where $\tilde{\mathbf{U}}=[\tilde{u}, \tilde{v}, \tilde{w}]$ is the dimensionless velocity field and $\hat{k}=\left[\begin{array}{lll}0 & 1 & 0\end{array}\right]$ is a unit vector indicating the directions in which gravity acts. All geometric dimensions (cylinder diameter, cylinder-to-cylinder spacing, upstream and downstream lengths) of the computational domain shown in Figures 5.2 and 5.4, were also made dimensionless by dividing through by the length scale $D_{0}$ :

$$
\tilde{D}_{0}=\frac{D_{0}}{D_{0}}=1, \quad \tilde{S}_{0}=\frac{S_{0}}{D_{0}}, \quad \tilde{H}_{d}, \tilde{H}_{u}=\frac{H_{d}, H_{u}}{D_{0}}
$$

\subsection{Objective function}

As shown in section 5.2.1, for a single scale structure, the cylinder-to-cylinder spacing is varied and for a multi scale structure (section 5.2.2), both the cylinder-to-cylinder spacing and the small cylinder diameter are varied. We are interested in the geometric 
configuration that maximizes the overall heat transfer between the cylinders and the surrounding fluid. The dimensionless quantity used to evaluate this configuration is the dimensionless heat transfer density rate. The heat transfer density rate is $q^{\prime \prime \prime}=$ $q^{\prime} / 2 D_{0}\left(D_{0}+S_{0}\right)$, where $q^{\prime}$ is the sum of the total heat transfer rate integrated over the surface of the cylinders:

$$
q^{\prime}=\sum_{i=1}^{d i} \frac{D_{0}}{2} \int_{0}^{2 \pi} k(\nabla T)_{s n} \mathrm{~d} \theta+\sum_{i=1}^{d j} \frac{D_{1}}{2} \int_{-\pi / 2}^{a \pi} k(\nabla T)_{s n} \mathrm{~d} \theta
$$

where $D_{0} / 2$ and $D_{1} / 2$ is the radius of the large diameter and small diameter cylinders respectively, $d i$ is the number of large diameter cylinders, $d j$ is the number of small diameter cylinders, $a=3 / 2$ or $a=1 / 2$ for a complete and half cylinder respectively. The subscript sn, in Eq. 5.9, denotes that gradient of $T$ is taken with respects to the normal direction to the cylinder surface. The corresponding dimensionless heat transfer density rate is:

$$
\tilde{q}=\frac{q^{\prime}}{2 D_{0}\left(D_{0}+S_{0}\right) k\left(T_{w}-T_{\infty}\right)}
$$

Thus the constrained optimisation problem can formally be written as:

$$
\begin{aligned}
& \underset{w . r . t . \mathbf{x}}{\operatorname{minimise}}: \\
& \qquad f(\mathbf{x})=-\tilde{q}(\mathbf{x}), \quad \mathbf{x}=\left[x_{1}, x_{2}\right]^{T} \in \mathbb{R}^{n}
\end{aligned}
$$

such that:

$$
\begin{aligned}
g_{1}(\mathbf{x}) & =-x_{1} \leq 0 \\
g_{2}(\mathbf{x}) & =-\left[\left(\frac{x_{1}+D_{0}}{2}\right)^{2}+\left(\frac{D_{0}-x_{2}}{2}\right)^{2}\right]^{0.5} \leq 0 \\
& =-\left[\left(x_{1}+D_{0}\right)^{2}+\left(D_{0}-x_{2}\right)^{2}\right]^{0.5} \leq 0
\end{aligned}
$$

where $x_{1}=S_{0}$ and $x_{2}=D_{1}$. The equality constraint $g_{1}(\mathbf{x})$ is added to Eq. 5.11 to ensure that the cylinder-to-cylinder spacing is greater than 0 and the second equality constraint $g_{2}(\mathbf{x})$ is added (only for the multi scale configuration) to ensure that the large diameter cylinders and small diameter cylinders do not overlap.

As mentioned in section 4.4, solving the optimisation problem (Eq. 5.11) only becomes practical for two or more design variables, hence only for a multi scale config- 
uration. Thus the optimisation problem (Eq. 5.11) was solved only for the multi scale configuration using the techniques described in section 4.4.3 and a parametrisation approach (as described in section 4.3) was used for the single scale configuration.

\subsection{Convergence criteria and mesh independence}

The numerical models being solved (section 5.2.1 and 5.2.1) were initialised with all internal variable fields being 0 (i.e. $\mathbf{U}^{0}=\mathbf{0}, P^{0}=0$ and $T^{0}=0$ ). Thus each simulation was viewed as a transient problem that reached steady-state at a certain time. The convergence criteria, used to solve the governing equations (cf. Fig. 3.1), at each time step was set based on the residual of each of the variable fields:

$$
R_{t}^{k}(\mathbf{U}) \leq 10^{-4}, \quad R_{t}^{k}(P) \leq 10^{-4}, \quad R_{t}^{k}(T) \leq 10^{-6}
$$

and the convergence criteria to terminate the simulation was evaluated based on the initial residual of each of the variable fields, i.e.:

$$
R_{t}^{0}(\mathbf{U}) \leq 10^{-4}, \quad R_{t}^{0}(P) \leq 10^{-4}, \quad R_{t}^{0}(T) \leq 10^{-6}
$$

In which $k$ was the iteration counter and $t$ was the time step.

The convergence criteria used to terminate the optimisation algorithm was:

$$
\left\|\mathbf{x}^{i}-\mathbf{x}^{i-1}\right\| \leq 10^{-3}
$$

where $\|\cdot\|$ is the Euclidean norm.

In order to obtain accurate heat flux results (Eq. 5.9), from all the simulations, the computational domain needed to be considered, namely the upstream and downstream lengths as well as the number of elements used to discretise the computational domain. The mesh design received special attention and was tested extensively in the range $10^{1} \leq R a \leq 10^{4}$, for $\tilde{\omega}_{0}=0$ and $\operatorname{Pr}=0.72$, with the grid varying from one geometric configuration to the next. A structured mesh, consisted of quadrilateral elements was used. The selected mesh arrangement shown in Fig. 5.5 (for both single scale and multi scale configuration) was uniform in the $\tilde{x}$ direction and double graded in the $\tilde{y}$ direction 
so as to put more elements near the cylinder surfaces to more accurately capture the behaviour of the boundary layers. The initial guess for the mesh size in the $\tilde{x}$ direction was chosen based on the boundary layer thickness scale $\delta_{T} \sim D_{0} R a^{(-1 / 4)}$.

The results from the mesh refinement and domain size studies has been split into two smaller sub-section: section 5.5.1 presents the results for the single scale configuration and section 5.5.2 presents the results for the multi scale configuration.

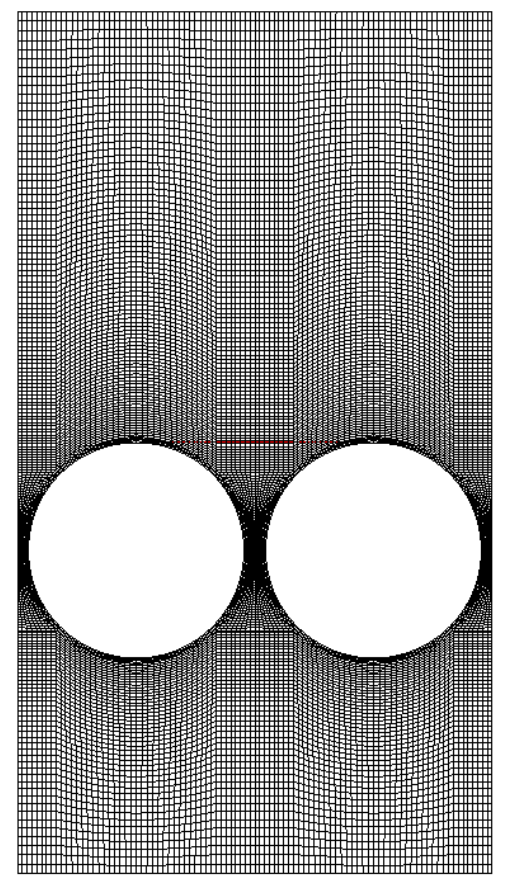

(a) Single scale mesh

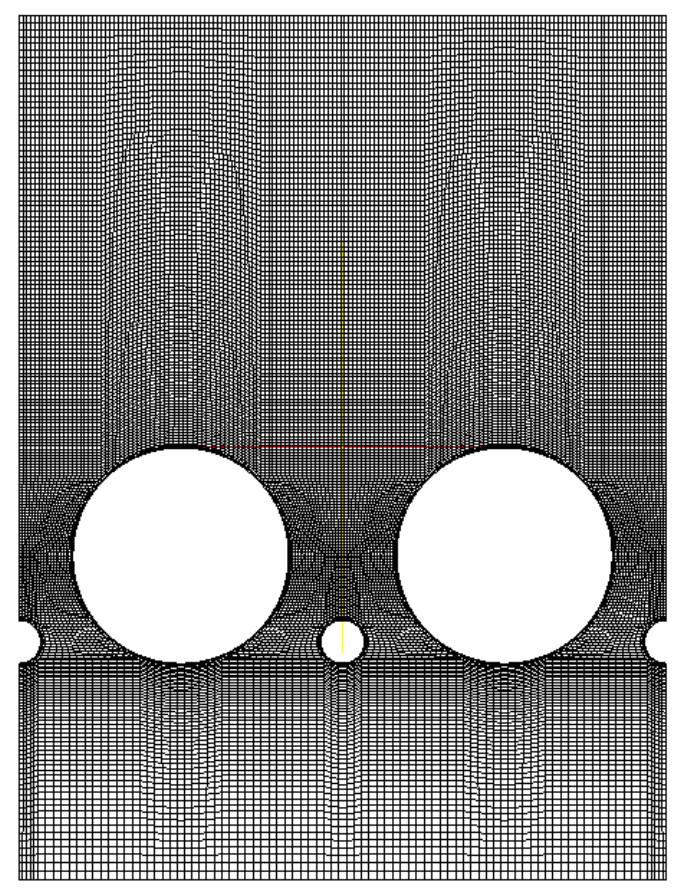

(b) Multi scale mesh

Figure 5.5: Illustration of the discretised flow domain.

\subsubsection{Single scale}

Table 5.1 shows a summary of the mesh refinement studies conducted for the range $10^{1} \leq R a \leq 10^{4}$ and $\tilde{\omega}_{0}=0$. This table shows the number of elements required per unit length at each Rayleigh number in order to achieve results independent of mesh size (varies by less than 1\%). For example the mesh refinement study shows, for $R a=10^{1}$, the heat transfer density rate was insensitive (varies by less than 1\%) to further mesh refinement when 16 elements per unit length were used. 
Both mesh independence and domain size independence was achieved in the same manner: by starting off with a low value for a given parameter and doubling that value until the result was insensitive to further refinement or doubling. Table 5.2 shows one example of how mesh independence was achieved for $R a=10^{3}, \tilde{S}_{0}=0.3, \tilde{\omega}_{0}=0$ and $\operatorname{Pr}=0.72$. It can be seen from this table that the heat transfer density rate was insensitive (varies by less than 1\%) to further mesh refinement when 48 elements per unit length were used.

\begin{tabular}{rr}
\hline$R a$ & Elements $/ L$ \\
\hline $10^{1}$ & 16 \\
$10^{2}$ & 48 \\
$10^{3}$ & 48 \\
$10^{4}$ & 100 \\
\hline
\end{tabular}

Table 5.1: Single scale mesh refinement summary: number of elements per unit length at each Rayleigh number $\left(\operatorname{Pr}=0.72\right.$ and $\left.\tilde{\omega}_{0}=0\right)$.

\begin{tabular}{lrr}
\hline Elements $/ L$ & $\tilde{q}$ & $\frac{\tilde{q}^{i}-\tilde{q}^{i+1}}{\tilde{q}^{i}}$ \\
\hline 12 & 6.899 & - \\
24 & 6.035 & 0.1365 \\
48 & 5.499 & 0.0887 \\
96 & 5.531 & 0.0058 \\
\hline
\end{tabular}

Table 5.2: Single scale mesh refinement study for $R a=10^{3}\left(\tilde{H}_{u}=1, \tilde{H}_{d}=2, \tilde{S}_{0}=0.3\right.$, $\tilde{\omega}_{0}=0$ and $\operatorname{Pr}=0.72$ ).

\begin{tabular}{lll}
\hline$R a$ & $\tilde{H}_{u}$ & $\tilde{H}_{d}$ \\
\hline $10^{1}$ & 1.5 & 3.5 \\
$10^{2}$ & 1.0 & 2.5 \\
$10^{3}$ & 1.0 & 2.0 \\
$10^{4}$ & 0.5 & 1.5 \\
\hline
\end{tabular}

Table 5.3: Single scale domain size summary: upstream and downstream lengths at each Rayleigh number $\left(\operatorname{Pr}=0.72\right.$ and $\left.\tilde{\omega}_{0}=0\right)$.

Table 5.3 shows a summary of the domain size study for the upstream and downstream lengths. For example the domain size study shows for $R a=10^{2}$, when $\tilde{H}_{u}=1.5$ and $\tilde{H}_{d}=2.5$, the heat transfer density rate was insensitive to further doubling of upstream and downstream lengths. 


\subsubsection{Multi scale}

Table 5.4 again shows the mesh refinement summary, for the number of elements required per unit length, for the multi scale configuration $\left(10^{1} \leq R a \leq 10^{4}\right.$ and $\left.\tilde{\omega}_{0}=0\right)$. Table 5.5 again shows one example of how mesh independence was achieved for $R a=10^{3}, \tilde{S}_{0}=0.5, \tilde{D}_{1}=0.2, \tilde{\omega}_{0}=0$ and $\operatorname{Pr}=0.72$.

\begin{tabular}{lr}
\hline$R a$ & Elements $/ L$ \\
\hline $10^{2}$ & 32 \\
$10^{3}$ & 48 \\
$10^{4}$ & 80 \\
\hline
\end{tabular}

Table 5.4: Multi scale mesh refinement summary: number of elements per unit length at each Rayleigh number $\left(\operatorname{Pr}=0.72\right.$ and $\left.\tilde{\omega}_{0}=0\right)$.

\begin{tabular}{lrr}
\hline Elements $/ L$ & $\tilde{q}$ & $\frac{\tilde{q}^{i}-\tilde{q}^{i+1}}{\tilde{q}^{i}}$ \\
\hline 12 & 6.604 & - \\
24 & 6.618 & 0.00219 \\
48 & 6.631 & 0.00199 \\
96 & 6.634 & 0.00041 \\
\hline
\end{tabular}

Table 5.5: Multi scale mesh refinement study for $R a=10^{3}\left(\tilde{H}_{u}=0.5, \tilde{H}_{d}=3.5\right.$, $\tilde{S}_{0}=0.5, \tilde{\omega}_{0}=0$ and $\left.\operatorname{Pr}=0.72\right)$.

\begin{tabular}{ccc}
\hline$R a$ & $\tilde{H}_{u}$ & $\tilde{H}_{d}=$ \\
\hline $10^{2}$ & 0.9 & $-4.57\left(\tilde{S}_{0}-\tilde{D}_{1}\right)+7.50$ \\
$10^{3}$ & 0.6 & $-5.11\left(\tilde{S}_{0}-\tilde{D}_{1}\right)+4.95$ \\
$10^{4}$ & 0.4 & $-5.31\left(\tilde{S}_{0}-\tilde{D}_{1}\right)+3.15$ \\
\hline
\end{tabular}

Table 5.6: Multi scale domain size summary: upstream and downstream lengths at each Rayleigh number $\left(\operatorname{Pr}=0.72\right.$ and $\left.\tilde{\omega}_{0}=0\right)$.

Table 5.6 shows a summary of the domain size study for the upstream and downstream lengths for $\operatorname{Pr}=0.72$. It was found that the heat transfer density rate is insensitive when the upstream length $\tilde{H}_{u}$ scales linearly as a function of the cylinderto-cylinder spacing minus the small cylinder diameter. For example the domain size study shows for $R a=10^{3}, \tilde{S}_{0}=0.5$, and $\tilde{D}_{1}=0.2$ that the heat transfer density rate 
is insensitive to further doubling of upstream and downstream lengths when $\tilde{H}_{u}=0.6$ and $\tilde{H}_{d}=-5.11\left(\tilde{S}_{0}-\tilde{D}_{1}\right)+4.95=3.42$.

From the results reported in section 5.5.1 and section 5.5.2 it can be seen that the mesh refinement and domain size studies were conducted with $\tilde{\omega}_{0}=0$ and it was assumed that the effect of cylinder rotation would not require a finer mesh and would not effect the upstream and downstream lengths.

\subsection{Conclusion}

This Chapter presented the numerical models in solving the fluid flow and temperature fields for both single scale and multi scale configurations. It was also shown how the governing equations are non-dimensionalised in order to report the results obtained in terms of dimensionless quantities. The objective function used to evaluate the maximum heat transfer density rate for the optimal geometric configurations was also presented in dimensionless form. The accuracy to which the governing equations are numerically solved as well as the accuracy to which the optimisation algorithm is solved was also presented. Finally the computational domain accuracy studies for achieving independent heat transfer density rates was presented. The results obtained from the numerical models presented in this Chapter is presented next in Chapter 6. 


\section{Chapter 6}

\section{Optimal geometric configuration results}

\subsection{Introduction}

This Chapter opens by using the method of scale analysis to predict the optimal structure results expected from the numerical simulations (Section 6.2). The results obtained from the numerical simulations are then presented in two subsection, Sections 6.3.1 and 6.3.2, for the single scale and multi scale structures respectively. Each of these two subsections is again split into smaller section. Sections 6.3.1.1 and 6.3.2.1 compare the results obtained from the numerical simulations for stationary cylinders against the results reported by Bello-Ochende and Bejan [22] for the single scale and multi scale structures respectively. Sections 6.3.1.2 and 6.3.2.2 then show the effect of rotation of the cylinders for the single scale and multi scale structures respectively.

\subsection{Scale analysis}

The optimal packing for a single scale structure, as shown in Fig. 5.1, is achieved when the cylinder-to-cylinder spacing is such that the thermal boundary layers of each cylinder just touch (shown in Fig. 6.1a). From Fig. 6.1b it can be seen how, for a 
multi scale structure, the flow structure of Fig. 6.1a is improved by inserting a smaller cylinder into the wedge-shaped region of the thermal boundary layer in order to utilise the region of fluid previously not used for heat transfer.

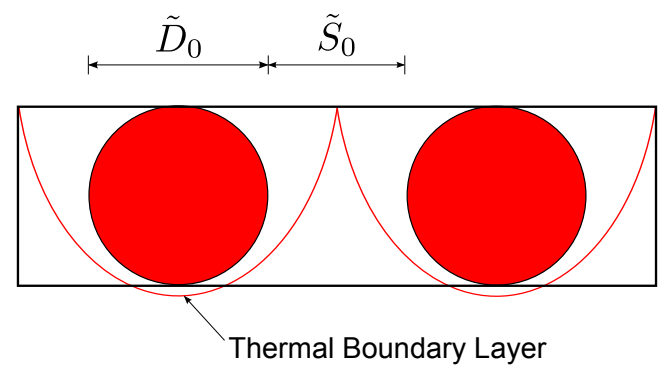

(a) Single scale configuration

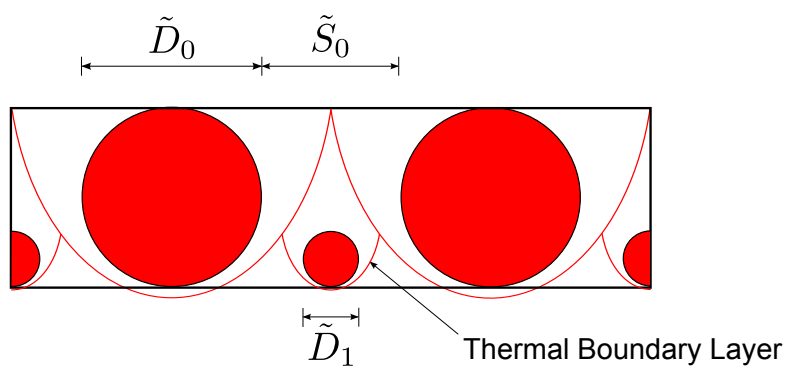

(b) Multi scale configuration

Figure 6.1: Illustration of the thermal boundary layer around a stationary cylinder

According to scale analysis, the thermal boundary layer of a cylinder with laminar natural convection flow and $\operatorname{Pr} \sim 1[14]$ has a thickness of order:

$$
\delta_{T} \sim D_{0} R a^{(-1 / 4)}
$$

By setting the thermal boundary layer thickness, $\delta_{T} \sim 2 S_{0}$ in Eq. 6.1, we find that:

$$
\tilde{S}_{0, o p t} \sim 2 R a^{(-1 / 4)}
$$

Using Eq. 6.2, the expected results for the single scale structure are shown in Table 6.1

The heat transfer density rate can be analysed based on the same scaling argument. The cylinder heat flux (heat transfer rate per unit area) scale is

$$
q^{\prime \prime} \sim k\left(T_{w}-T_{\infty}\right) / \delta_{T}
$$

where $\delta_{T} \sim D_{0} R a^{(-1 / 4)}$. Because $S_{0}<D_{0}$ (cf. Eq. 6.1) for $R a \gg 1$, the heat transfer density rate is $q^{\prime \prime \prime} \sim q^{\prime \prime} / D_{0}$, such that the dimensionless heat transfer density rate becomes:

$$
\tilde{q} \sim \frac{q^{\prime \prime \prime} D_{0}^{2}}{k\left(T_{w}-T_{\infty}\right)} \sim R a^{(-1 / 4)}
$$




\begin{tabular}{rc}
\hline$R a$ & $\tilde{S}_{0} \sim$ \\
\hline 10 & 1.125 \\
100 & 0.632 \\
1000 & 0.356 \\
10000 & 0.200 \\
100000 & 0.112 \\
\hline
\end{tabular}

Table 6.1: Scale analysis for the optimal cylinder-to-cylinder spacing for a single scale structure.

\subsection{Results}

The flow and temperature fields were simulated in a large number of configurations, in order to determine the effect of the cylinder-to-cylinder spacing on the heat transfer density rate at each flow regime for each cylinder rotation speed. The Rayleigh number range considered is $10^{1} \leq R a \leq 10^{4}$ and the cylinder rotation speed range considered is $0 \leq \tilde{\omega}_{0} \leq 10^{4}$.

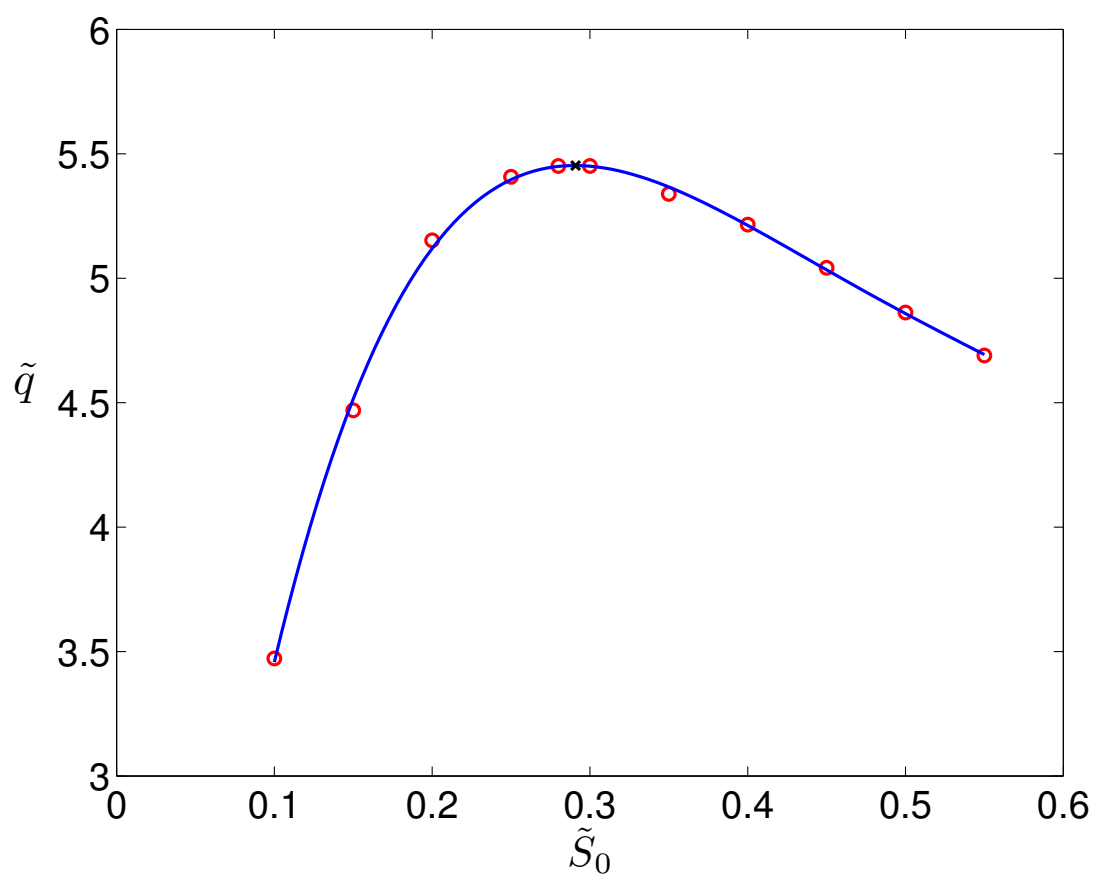

Figure 6.2: The maximization of the heat transfer density rate for the assemble shown in Fig. 5.1 for $R a=10^{3}, \operatorname{Pr}=0.72$ and $\tilde{\omega}_{0}=0$. 


\subsubsection{Single scale}

\subsubsection{Stationary cylinders}

Figure 6.2 shows that the heat transfer density rate is optimal when $\tilde{S}_{0}$ has a certain value, when there is no cylinder rotation. The optimal spacing, shown in Fig. 6.2, for $R a=10^{3}, \operatorname{Pr}=0.72$ and $\tilde{\omega}_{0}=0$ is $\tilde{S}_{0, o p t}=0.291$. There is a $0.6 \%$ difference when comparing this optimal spacing with that reported by Bello-Ochende and Bejan [22] for a single row of cylinders.

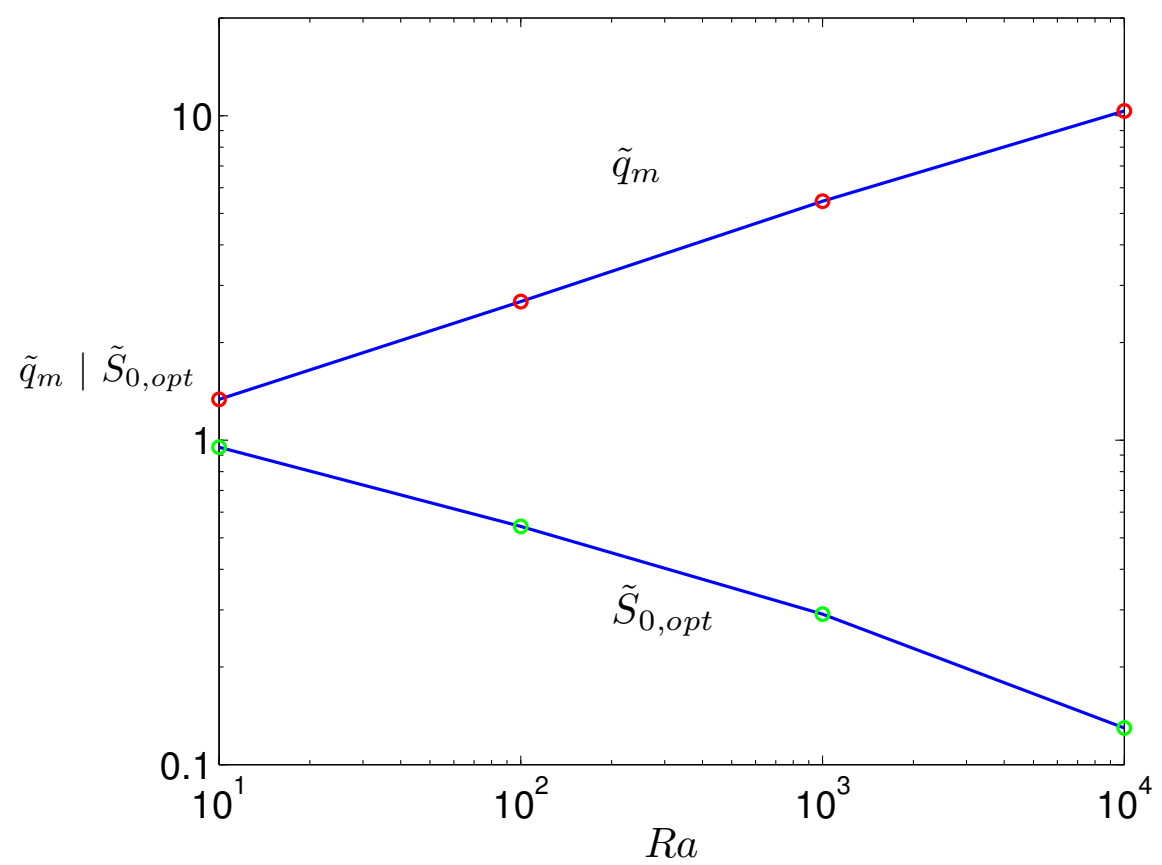

Figure 6.3: The optimal cylinder-to-cylinder spacings and corresponding heat transfer density rates for a row of cylinders shown in Fig. 5.1 for $\operatorname{Pr}=0.71, \tilde{\omega}_{0}=0$ and $10^{1} \leq R a \leq 10^{4}$.

The optimal cylinder-to-cylinder spacings and corresponding maximum heat transfer density rates for $10^{1} \leq R a \leq 10^{4}, \operatorname{Pr}=0.72$ and $\tilde{\omega}_{0}=0$ are summarized in Fig. 6.3. The optimal cylinder-to-cylinder spacings (from Fig. 6.3) can be correlated by the power law, within $0.25 \%$ :

$$
\tilde{S}_{0, o p t}=1.76 R a^{(-0.26)}
$$

Equation. 6.5 is anticipated well by the scale analysis argument, cf. Eq. 6.2. 
This correlation also compares well with the power law correlation proposed by Bello-Ochende and Bejan [22]: $\tilde{S}_{0, \text { opt }}=1.32 R a^{(-0.22)}$. For $10^{3} \leq R a \leq 10^{5}$, there is a $1 \%$ difference $\left(R a=10^{3}\right), 8 \%$ difference $\left(R a=10^{4}\right)$ and $16 \%$ difference $\left(R a=10^{5}\right)$. The corresponding maximum heat transfer density rates, reported in Fig. 6.3, can be correlated by the power law, within $0.05 \%$ :

$$
\tilde{q}_{m}=0.72 R a^{0.29}
$$

Again Equation 6.6 is anticipated well by the scale analysis argument, cf. Eq. 6.4. This correlation also compares well with the power law correlation proposed by BelloOchende and Bejan [22]: $\tilde{q}_{m}=0.65 R a^{0.30}$. For $10^{3} \leq R a \leq 10^{5}$, there is a $3 \%$ difference $\left(R a=10^{3}\right), 1 \%$ difference $\left(R a=10^{4}\right)$ and $1 \%$ difference $\left(R a=10^{5}\right)$.

\subsubsection{Rotating cylinders}

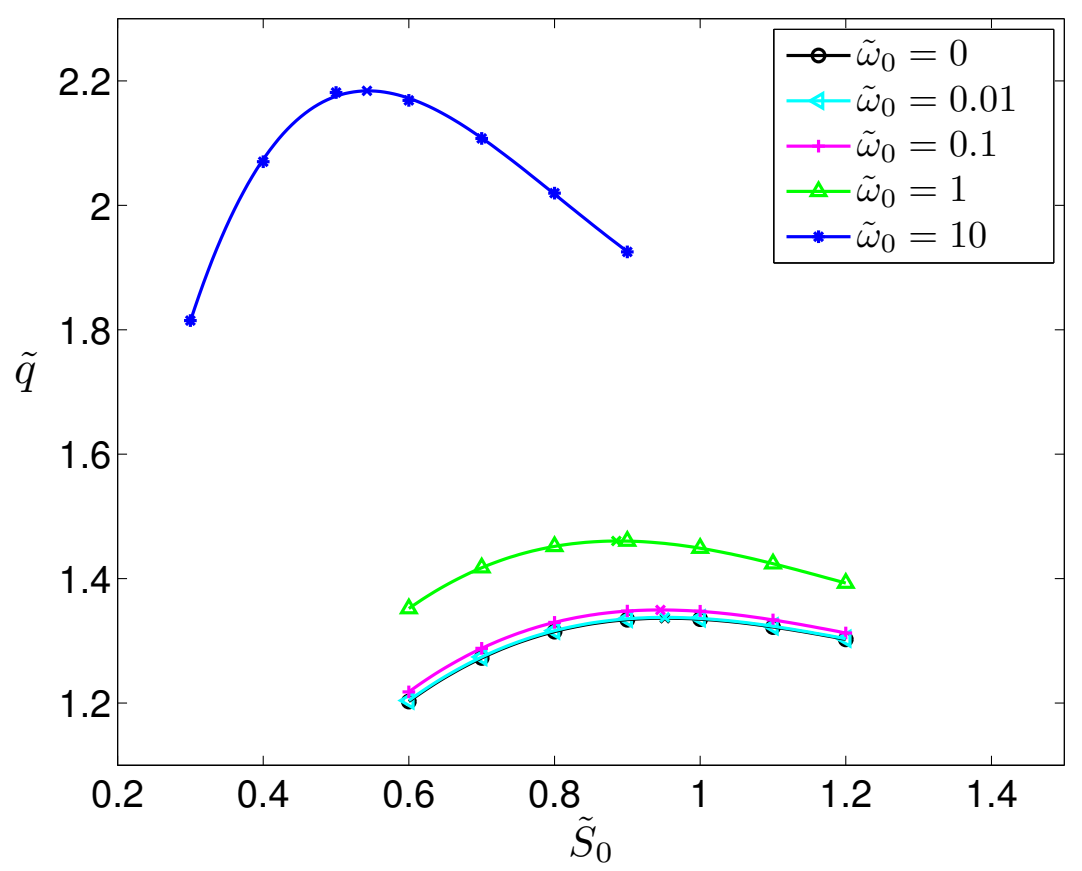

Figure 6.4: The maximization of the heat transfer density rate for the cylinders shown in Fig. 5.1 for $R a=10^{1}$ and $\operatorname{Pr}=0.71$ at different rotation speeds.

Figure 6.4 shows the optimal cylinder-to-cylinder spacing and the heat transfer density rate for $R a=10^{1}$ and $\operatorname{Pr}=0.72$ at different cylinder rotation speeds $(0 \leq$ $\left.\tilde{\omega}_{0} \leq 10\right)$. The optimal cylinder-to-cylinder spacing decreases and the maximum heat 
transfer density rate increases as the cylinder rotational speed is increased. There is a $63 \%$ increase in the maximum heat transfer density rate for the optimized structure when the cylinder rotation speed is increased from stationary to $\tilde{\omega}_{0}=10$.

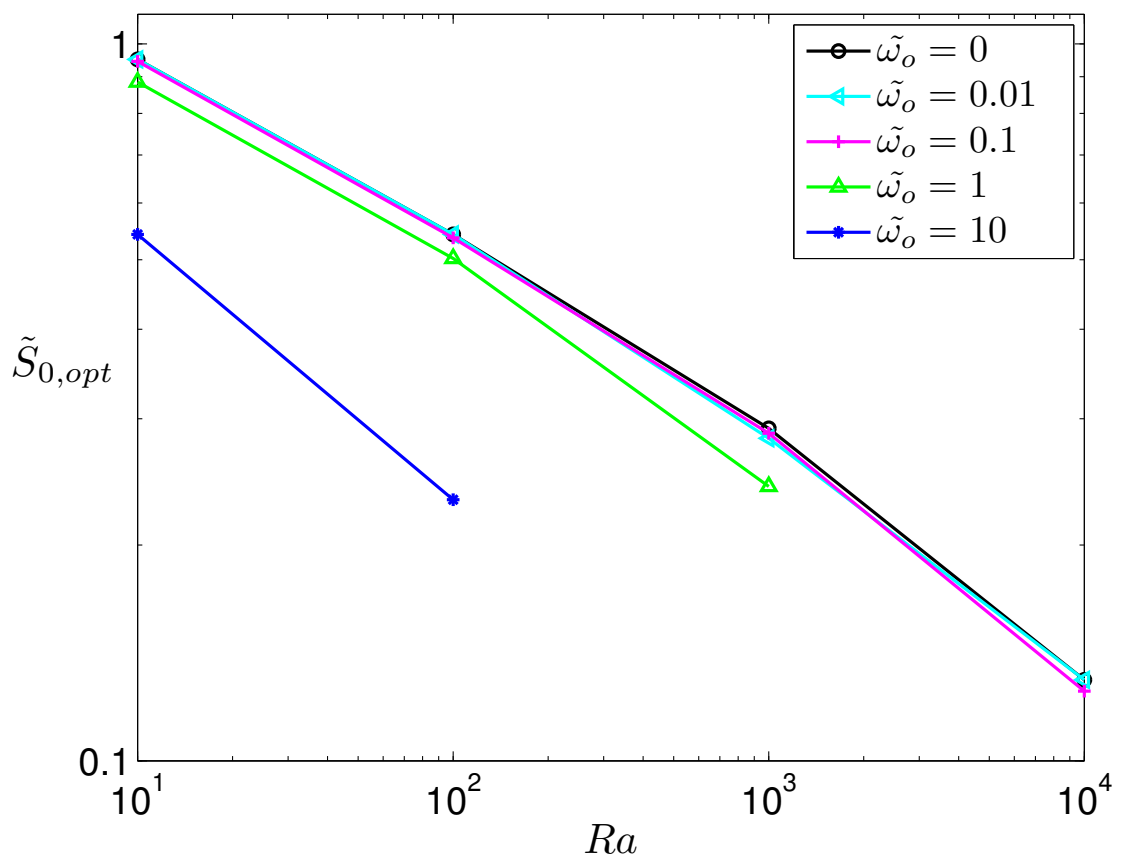

Figure 6.5: The optimal cylinder-to-cylinder spacings for a row of rotating cylinders shown in Fig. 5.1 for $\operatorname{Pr}=0.71$.

The optimal cylinder-to-cylinder spacings for $10^{1} \leq R a \leq 10^{4}, \operatorname{Pr}=0.72$ and $0 \leq \tilde{\omega}_{0} \leq 10$ are summarized in Fig. 6.5. A cylinder rotational speed of $\tilde{\omega}_{0}=0.01$ has no impact on the optimal cylinder-to-cylinder spacing when compared to that of stationary cylinders. The optimal cylinder-to-cylinder spacing is reduced by $3.5 \%$ for $R a=10^{4}$ and by less than $1.5 \%$ for $10^{1} \leq R a \leq 10^{3}$ when the cylinder rotational speed is increased from stationary to $\tilde{\omega}_{0}=0.1$. There is a $57 \%$ decrease in the optimal cylinder-to-cylinder spacing for $R a=10^{2}$ when the cylinder rotation speed is increased from stationary to $\tilde{\omega}_{0}=10$ and a $17 \%$ decrease in the optimal cylinder-to-cylinder spacing for $R a=10^{3}$ when the cylinder rotation speed is increased from stationary to $\tilde{\omega}_{0}=1$.

When the Rayleigh number is equal to $10^{3}$ and the cylinder rotational speed is greater than 1, the results become non-physical because the laminar model of the flow collapses due to a wake and consequent turbulence, which dominates the flow behind the rotating cylinders. This is also the case when the Rayleigh number is equal to $10^{4}$ 
and the cylinder rotational speed is greater than 0.1. The optimal cylinder-to-cylinder spacings, reported in Fig. 6.5, can be correlated by a power law, within $0.33 \%$, of the form $\tilde{S}_{o, \text { opt }}=C R a^{n}$ where $C$ and $n$ are given in Table 6.2 , for the cylinder rotation speeds $0.01,0.1,1$ and 10 respectively. These individual correlations can be simplified into one power law correlation, with an error of less than $1 \%$ :

$$
\tilde{S}_{0, o p t}=-0.05 \tilde{\omega}_{0}^{0.86}+1.69 R a^{-0.25}
$$

The maximum heat transfer density rates for $10^{1} \leq R a \leq 10^{4}, \operatorname{Pr}=0.72$ and $0 \leq \tilde{\omega}_{0} \leq 10$ are summarized in Fig. 6.6. A cylinder rotational speed of $\tilde{\omega}_{0}=0.01$ has less than a $0.4 \%$ increase on the maximum heat transfer density rate when compared to that of stationary cylinders. The maximum heat transfer density rate is increase by $2.5 \%$ for $R a=10^{4}$ and by less than $1.5 \%$ for $10^{1} \leq R a \leq 10^{3}$ when the cylinder rotational speed is increased from stationary to $\tilde{\omega}_{0}=0.1$. There is a $46 \%$ increase in the maximum heat transfer density rate for $R a=10^{2}$ when the cylinder rotation speed is increased from stationary to $\tilde{\omega}_{0}=10$ and a $13 \%$ increase in the maximum heat transfer density rate for $R a=10^{3}$ when the cylinder rotation speed is increased from stationary to $\tilde{\omega}_{0}=1$.

\begin{tabular}{rcccc}
\hline & \multicolumn{2}{c}{$\tilde{S}_{0, \text { opt }}$} & \multicolumn{2}{c}{$\tilde{q}_{m}$} \\
\cline { 2 - 5 }$\tilde{\omega}_{0}$ & $C$ & $n$ & $C$ & $n$ \\
\hline 0.01 & 1.77 & -0.27 & 0.72 & 0.29 \\
0.1 & 1.75 & -0.27 & 0.72 & 0.29 \\
1 & 1.65 & -0.27 & 0.70 & 0.31 \\
10 & 1.27 & -0.37 & 1.22 & 0.25 \\
\hline
\end{tabular}

Table 6.2: Coefficients for the power law correlation of the form $C R a^{n}$ for $\tilde{S}_{0, \text { opt }}$ and $\tilde{q}_{m}$.

The maximum heat transfer density rates, reported in Fig. 6.6, can be correlated by a power law, within $0.05 \%$, of the form $\tilde{q}_{m}=C R a^{n}$ where $C$ and $n$ are given in Table 6.2, for the cylinder rotation speeds 0.01, 0.1, 1 and 10 respectively. Again these individual correlations can be simplified into one power law correlation, with an error of less than $1 \%$ :

$$
\tilde{q}_{m}=0.32 \tilde{\omega}_{0}^{0.49}+0.71 R a^{0.29}
$$




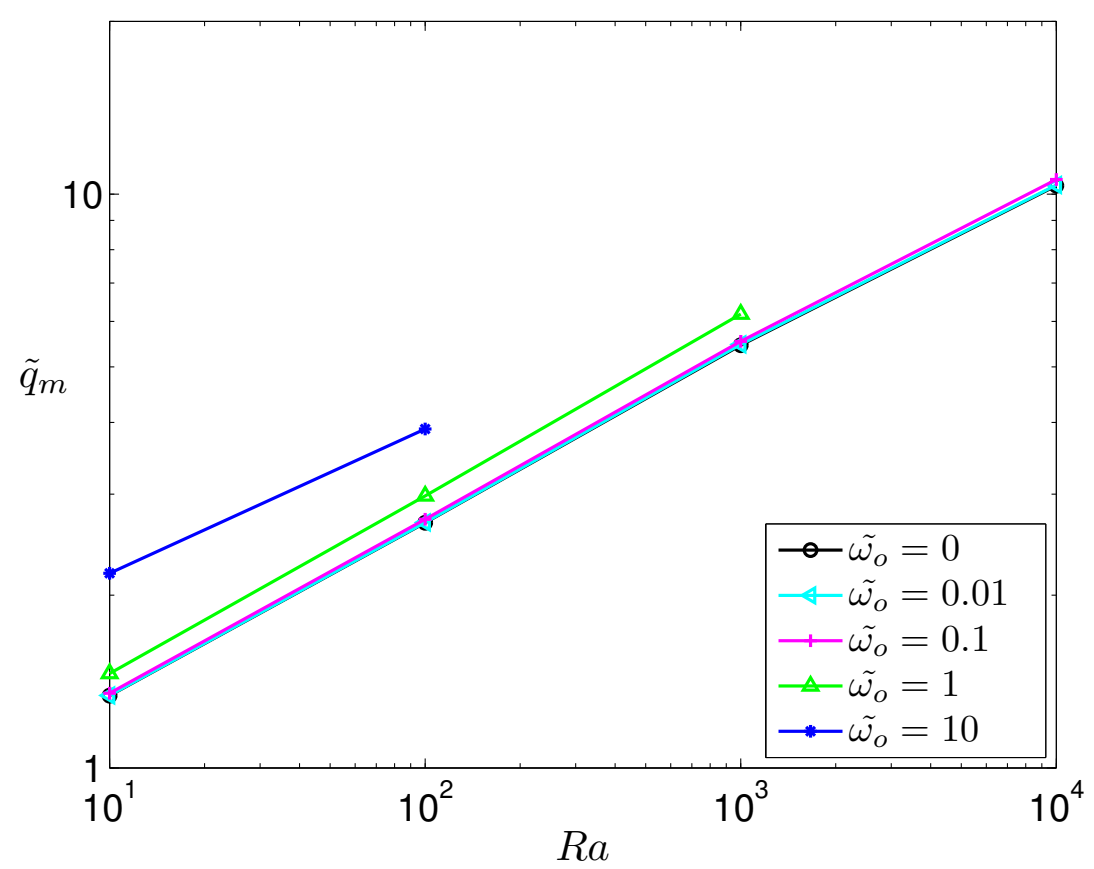

Figure 6.6: The maximum heat transfer density rates for a row of rotating cylinders shown in Fig. 5.1 for $\operatorname{Pr}=0.71$.

Figure 6.7 shows the effect of cylinder rotational speed on the thermal boundary layer for a row of rotating cylinders shown in Fig. 5.1 for $R a=10^{2}, \operatorname{Pr}=0.72$ and $S o=0.5$. Figure 6.7 a shows, for $\tilde{\omega}_{0}=0$, that the thermal boundary layer between two consecutive cylinders touches near the centreline of the cylinders. Figure 6.7a shows, for $\tilde{\omega}_{0}=10$, that the thermal boundary layer between two consecutive cylinders (where the cylinder rotation aids the flow direction) is extended and touches just past the top of the cylinders. Similarly, the thermal boundary layer between two consecutive cylinders (where the cylinder rotation opposes the flow direction) remains relatively unchanged.

The results presented in this section can also be compared to the work of Joucaviel et al. [34] in the sense that the trends in this thesis and in the work of Joucaviel et al. are in agreement: the maximum heat transfer density rate increased and the optimal cylinder-to-cylinder spacing decreased as the cylinder rotation was increased. 


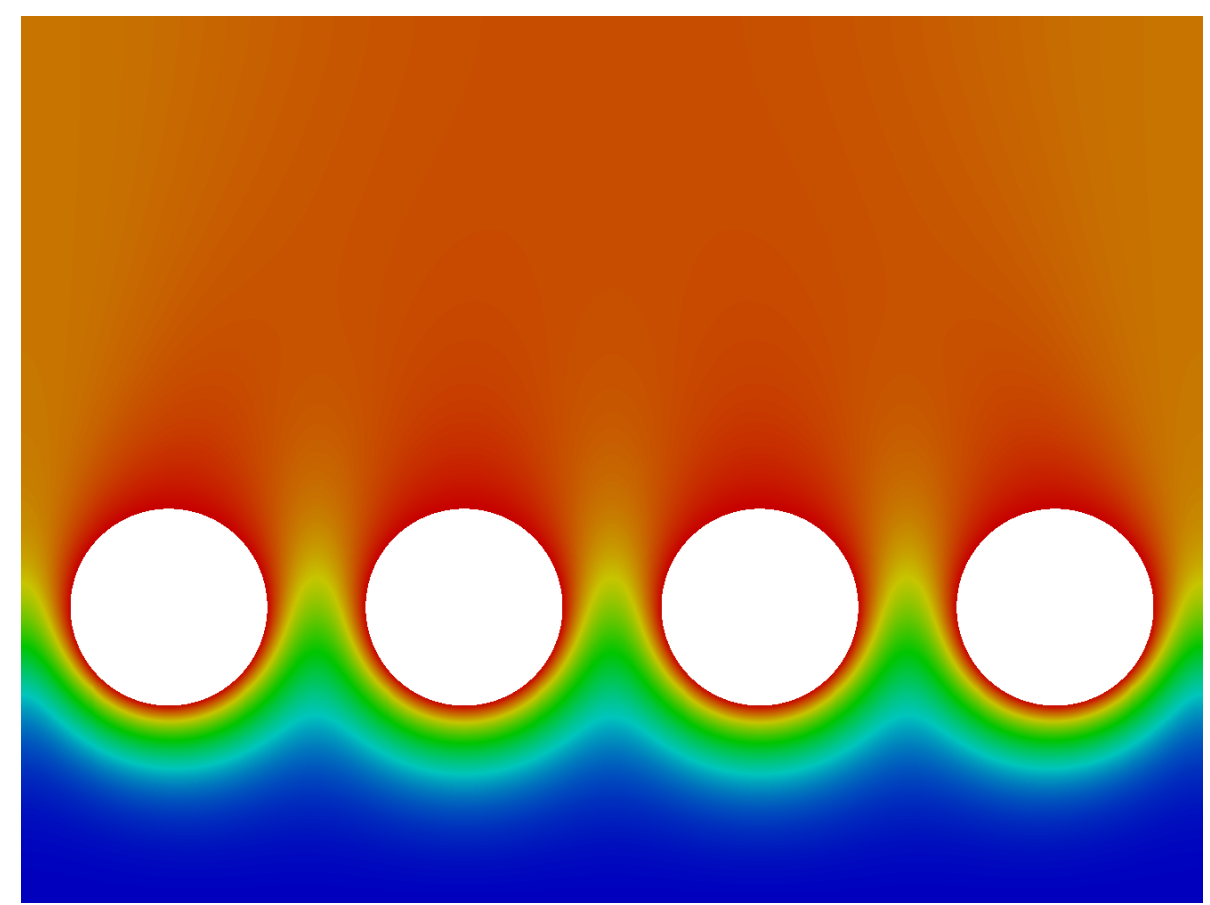

(a) $\tilde{\omega}_{0}=0$

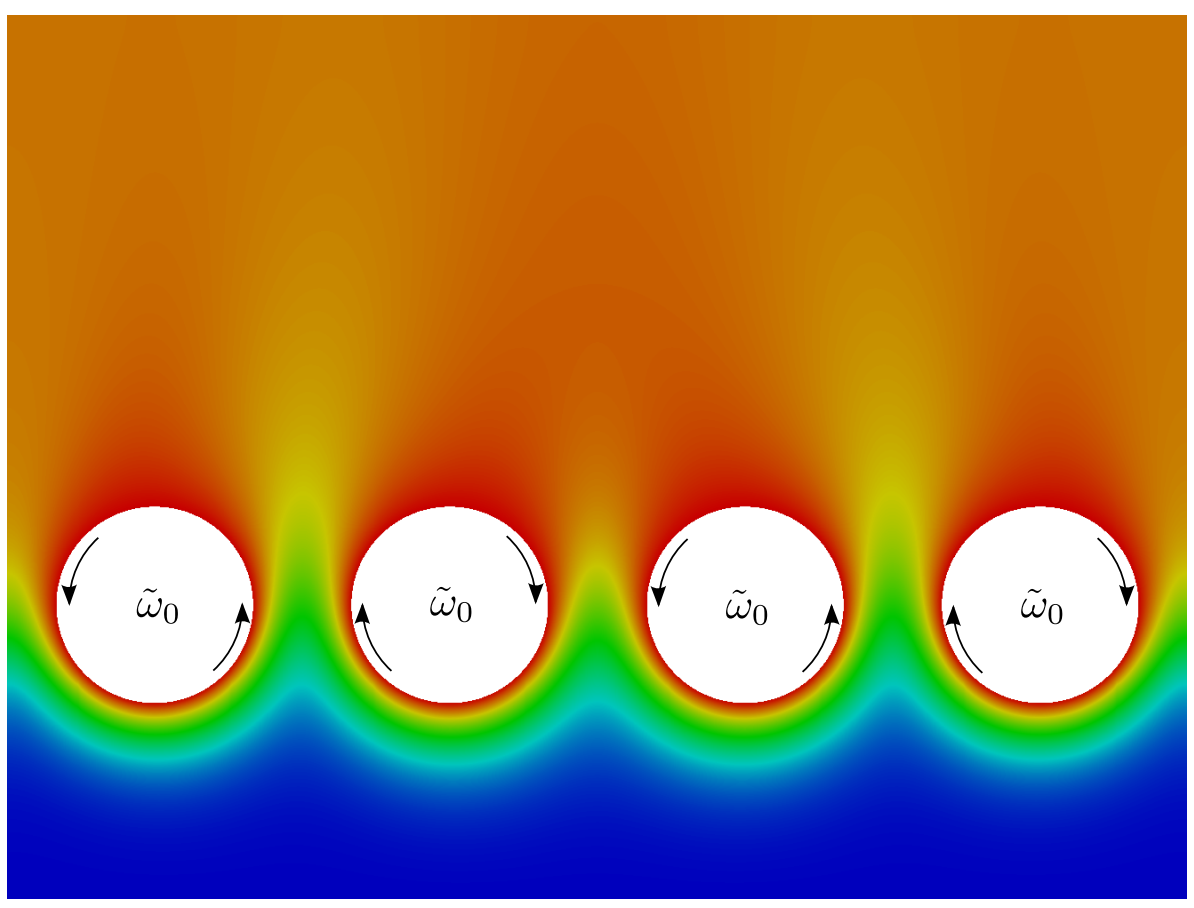

(b) $\tilde{\omega}_{0}=10$

Figure 6.7: The effect of cylinder rotational speed on the thermal boundary layer for a row of rotating cylinders shown in Fig. 5.1 


\subsubsection{Multi scale}

\subsubsection{Stationary cylinders}

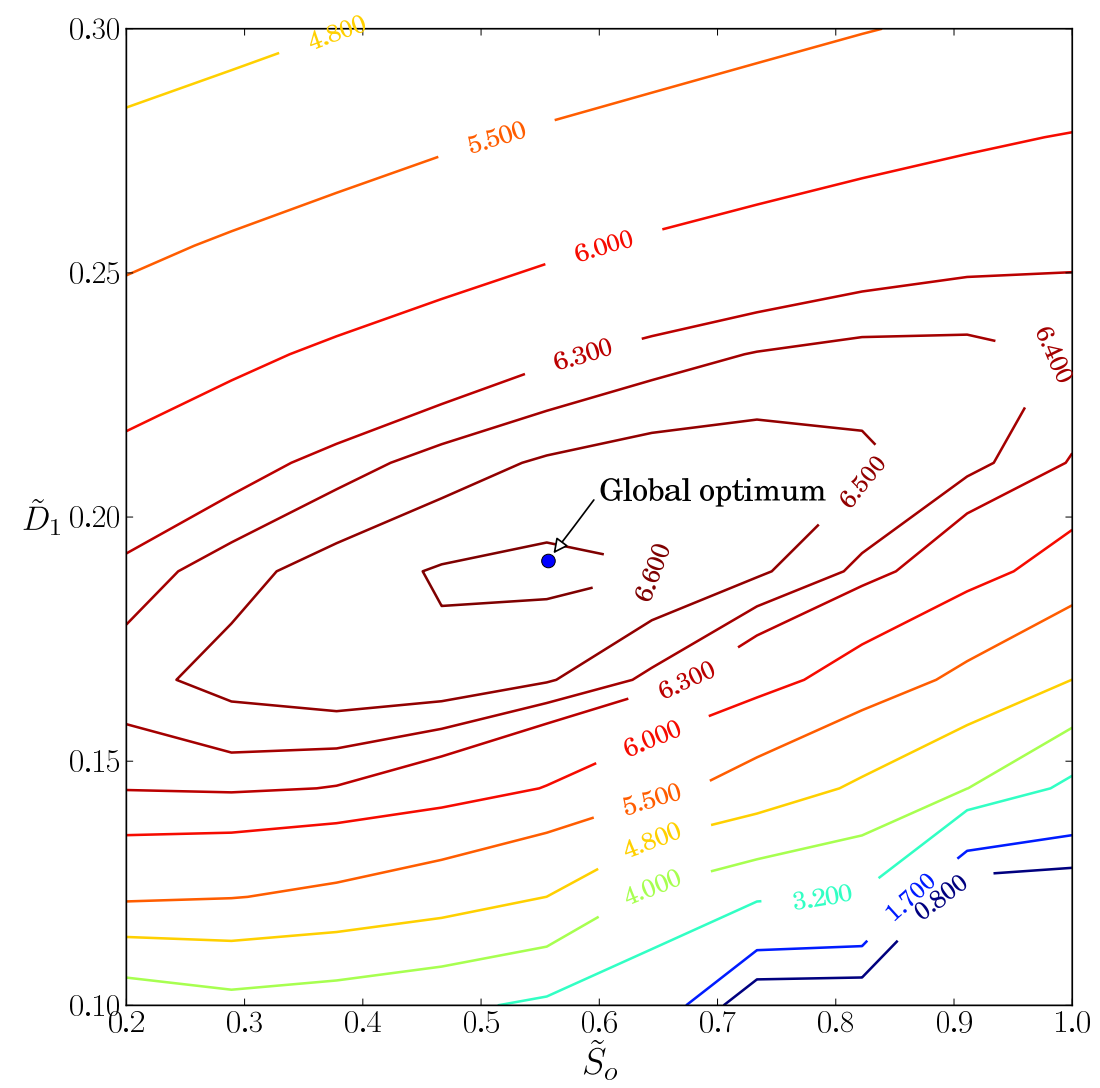

Figure 6.8: Contour plot of the heat transfer density rates for a row of rotating cylinders shown in Fig. 5.3 for $R a=10^{3}$ and $\operatorname{Pr}=0.71$.

As discussed in Chaper 4 and Chapter 5, an optimisation routine was used to determine the optimal geometric configuration for the maximum heat transfer density rate. The accuracy of results obtained from the optimisation routine need to be considered. Figure 6.8 shows a contour plot for the heat transfer density rate for and range of cylinder-to-cylinder spacings $\left(\tilde{S}_{0}\right)$ and small cylinder diameters $\left(\tilde{D}_{1}\right)$ for $R a=10^{3}$, $\operatorname{Pr}=0.72$ and $\tilde{\omega}_{0}=0$. The optimum configuration obtained from the optimisation routine is also shown in Fig. 6.8 (as the "Global optimum") and it can be seen that this result is very feasible and compares well with the data collected. It is also worth mentioning that the heat transfer density rate (objective function) is very well behaved, in 
the sense that it can be considered to be smooth, continuous and have no local optima.

The feasibility of the result obtained from the optimisation routine is again shown in Fig. 6.9. This figure shows that the cylinder-to-cylinder spacing can be optimized, for maximum heat transfer density rate, for each small cylinder diameter. It can further be seen, from Fig. 6.9, that there exists a global optimum heat transfer density rate when $\tilde{S}_{0}$ and $\tilde{D}_{1}$ have a certain value, when there is no cylinder rotation. The global optimum shown in Fig. 6.9, for $\operatorname{Ra}=10^{3}, \operatorname{Pr}=0.72$ and $\tilde{\omega}_{0}=0$, is found when $\tilde{S}_{0, o p t}=0.557$ and $\tilde{D}_{1, o p t}=0.191$. There is a $14.8 \%$ and $4.5 \%$ difference when comparing this optimal cylinder-to-cylinder spacing and small cylinder diameter, respectively, with that reported by Bello-Ochende and Bejan [22] for a multi scale configuration of cylinders with two length scales.

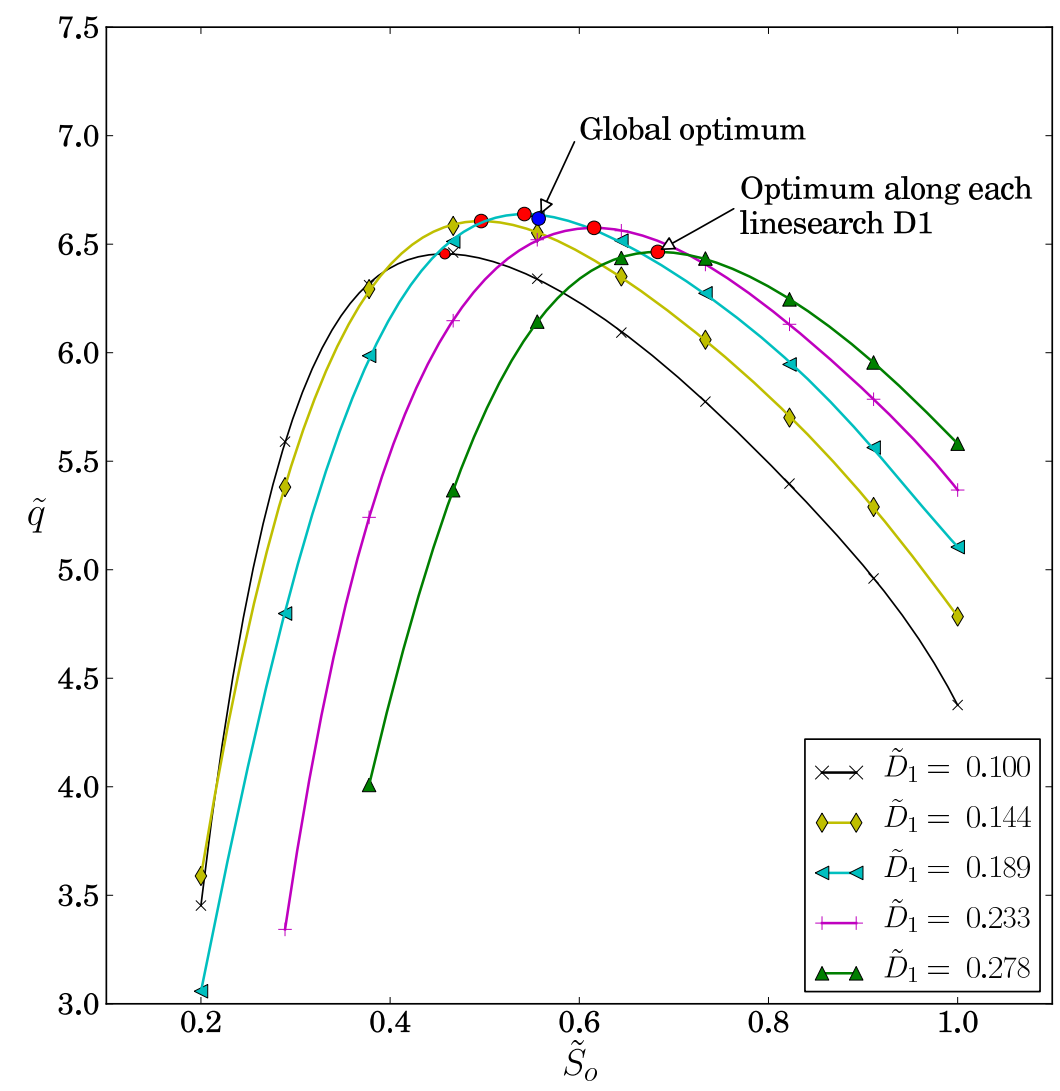

Figure 6.9: The maximum heat transfer density rates for a row of rotating cylinders shown in Fig. 5.3 for $R a=10^{3}$ and $\operatorname{Pr}=0.71$.

The optimal cylinder-to-cylinder spacings and corresponding maximum heat trans- 
fer density rates for $10^{2} \leq R a \leq 10^{4}, \operatorname{Pr}=0.72$ and $\tilde{\omega}_{0}=0$ are summarized in Fig. 6.10. The optimal cylinder-to-cylinder spacings (from Fig. 6.10) can be correlated by the power law, within $0.27 \%$ :

$$
\tilde{S}_{0, o p t}=2.113 R a^{(-0.205)}
$$

This correlation compares reasonably well with the power law correlation proposed by Bello-Ochende and Bejan [22]: $\tilde{S}_{0, o p t}=2.218 R a^{(-0.22)}$. For $10^{3} \leq R a \leq 10^{5}$, there is a $5.67 \%$ difference $\left(R a=10^{3}\right), 9.38 \%$ difference $\left(R a=10^{4}\right)$ and $13.24 \%$ difference $\left(R a=10^{5}\right)$.

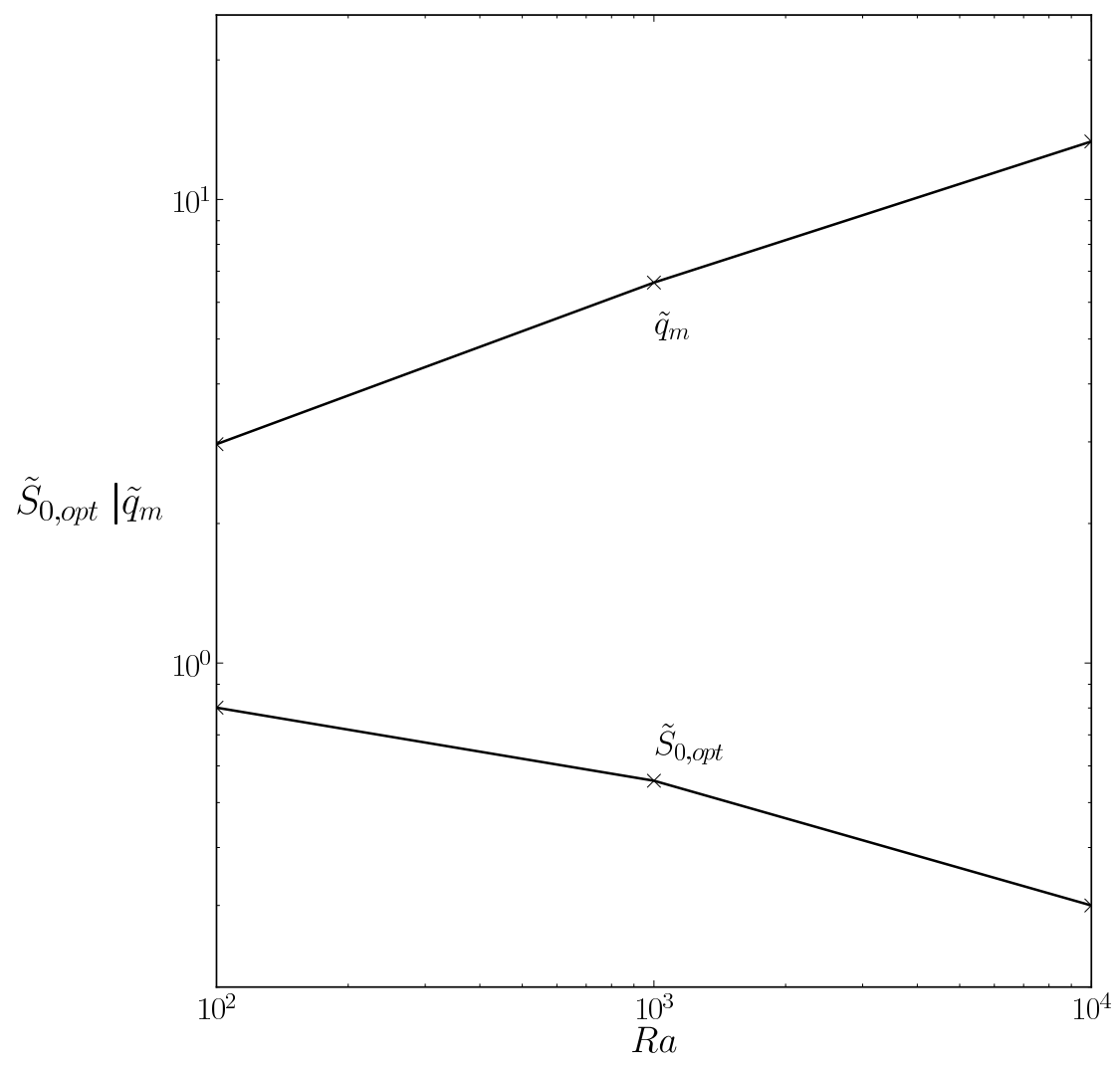

Figure 6.10: The optimal cylinder-to-cylinder spacings and corresponding heat transfer density rates for a row of cylinders shown in Fig. 5.3 for $\operatorname{Pr}=0.72, \tilde{\omega}_{0}=0$ and $10^{2} \leq R a \leq 10^{4}$.

The corresponding maximum heat transfer density rates, reported in Fig. 6.10, can 
be correlated by the power law, within $5.9 \%$ :

$$
\tilde{q}_{m}=0.698 R a^{0.321}
$$

This correlation also compares well with the power law correlation proposed by BelloOchende and Bejan [22]: $\tilde{q}_{m}=0.85 R a^{0.30}$. For $10^{3} \leq R a \leq 10^{5}$, there is a $5.06 \%$ difference $\left(R a=10^{3}\right), 0.36 \%$ difference $\left(R a=10^{4}\right)$ and $4.58 \%$ difference $\left(R a=10^{5}\right)$.

\subsubsection{Rotating cylinders}

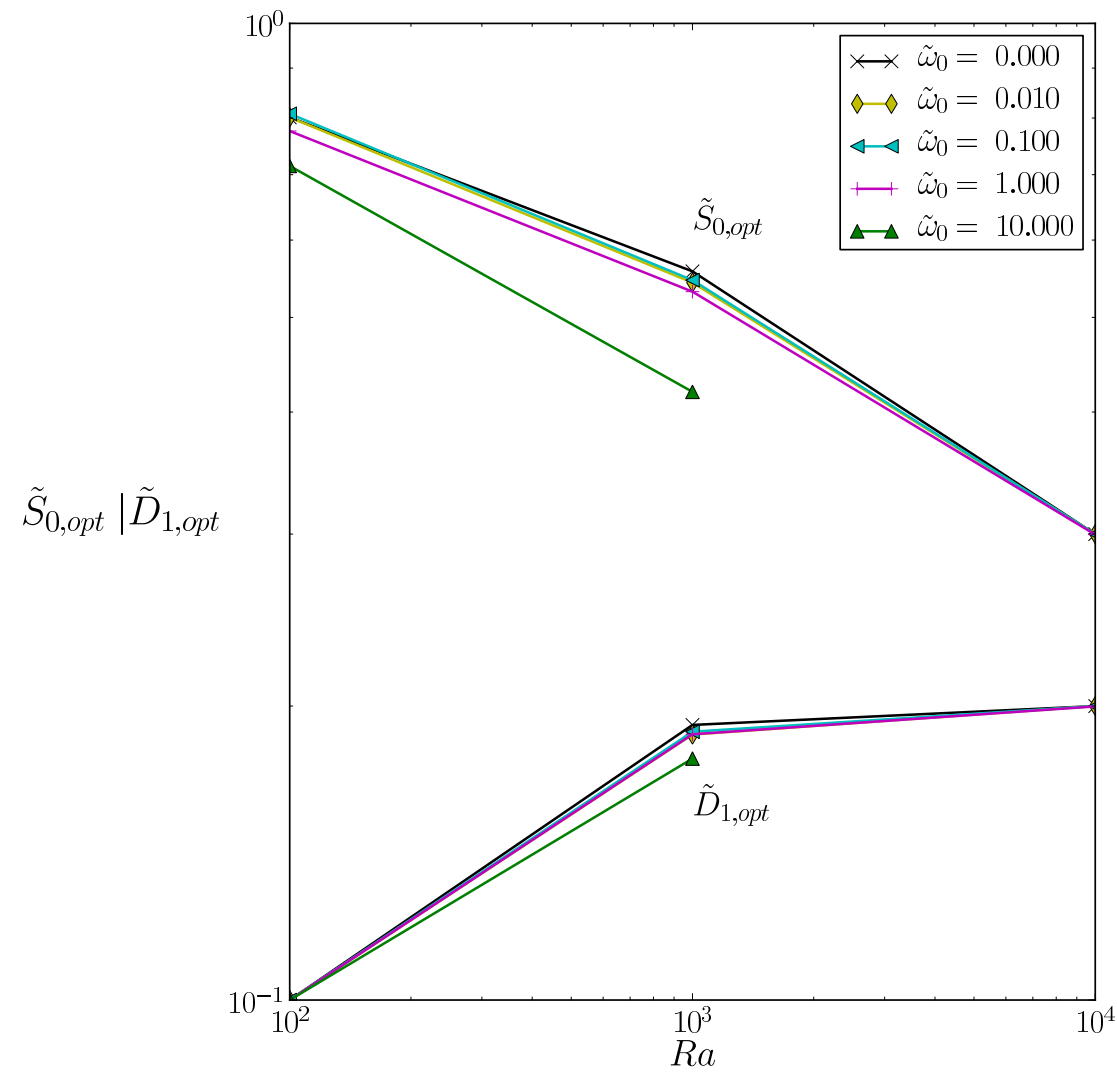

Figure 6.11: The optimal cylinder-to-cylinder spacings and small cylinder diameter for a row of rotating cylinders shown in Fig. 5.3 for $\operatorname{Pr}=0.72$.

The optimal cylinder-to-cylinder spacings and optimal small cylinder diameters for $10^{2} \leq R a \leq 10^{4}, \operatorname{Pr}=0.72$ and $0 \leq \tilde{\omega}_{0} \leq 10$ are summarized in Fig. 6.11. This figure shows that any cylinder rotation in the range $0 \leq \tilde{\omega}_{0} \leq 1$ has very little improvement 
on the the optimal geometric configuration for a multi scale structure. There is a maximum decrease in the optimal cylinder-to-cylinder spacing of $3.1 \%, 4.7 \%$ and $0 \%$ for the $R a$ numbers $10^{2}, 10^{3}$ and $10^{4}$ respectively, when the cylinder rotation speed is increased from stationary to $\tilde{\omega}_{0}=1$. It is also shown that, for a cylinder rotation speed of $\tilde{\omega}_{0}=10$, there is a decrease in the optimal cylinder-to-cylinder spacing of $10.74 \%$ and $24.57 \%$ for the $R a$ numbers $10^{2}$ and $10^{3}$ and respectively. When the Rayleigh number is equal to $10^{4}$ and the cylinder rotational speed is greater than 1 , the results become non-physical because the laminar model of the flow collapses due to a wake and consequent turbulence, which dominates the flow behind the rotating cylinders.

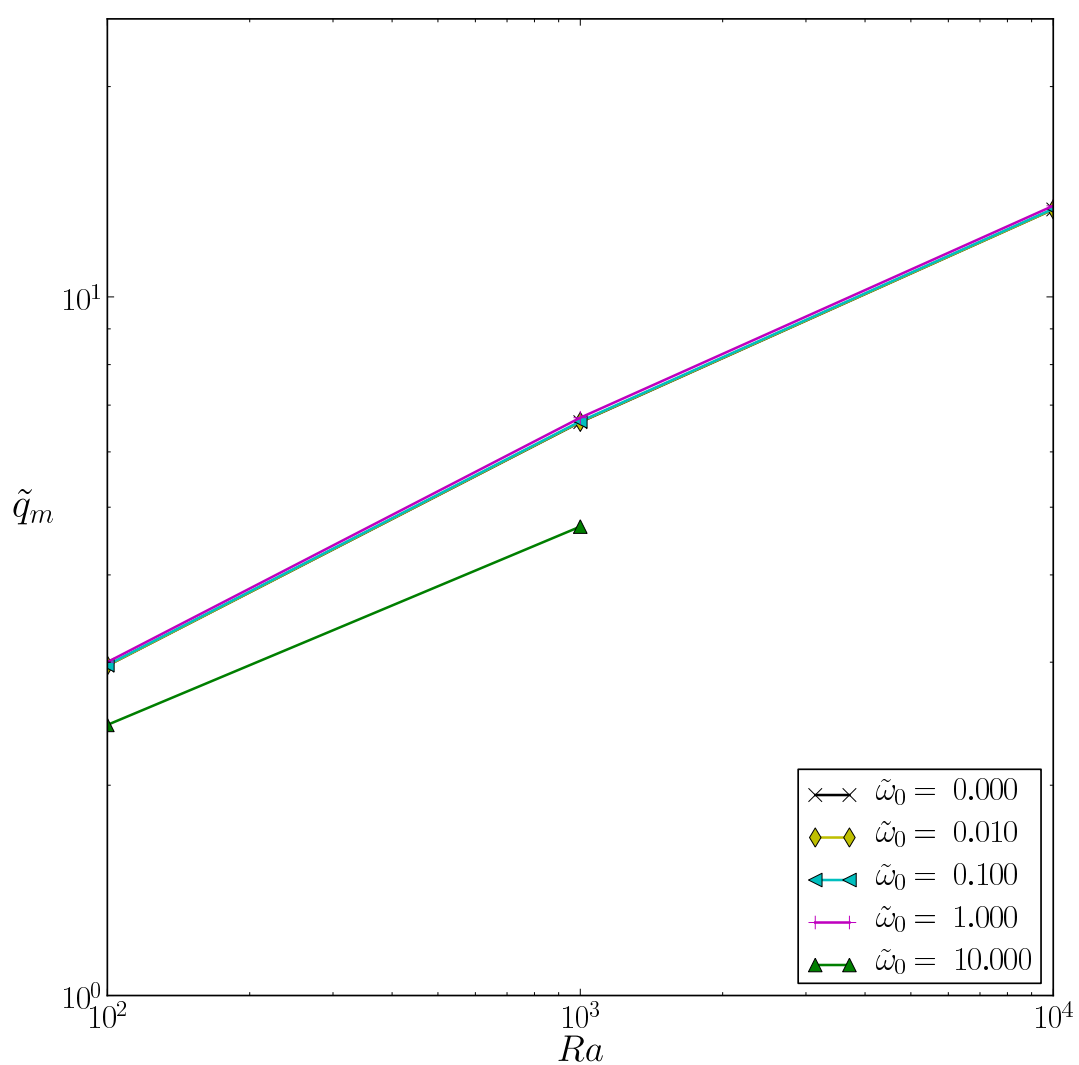

Figure 6.12: The maximum heat transfer density rates for a row of rotating cylinders shown in Fig. 5.3 for $\operatorname{Pr}=0.72$.

The optimal small cylinder diameters, shown in Fig. 6.11, is 0.2 for all cylinder rotation speeds for $R a=10^{4}$ and 0.19 for all cylinder rotation speeds (with the exception of $\left.\tilde{\omega}_{0}=10\right)$ for $R a=10^{3}$. This result compares well with the result proposed by Bello-Ochende and Bejan [22]: $\tilde{D}_{1, o p t}=0.2$. For the Rayleigh number $R a=10^{2}$ 
the optimal small cylinder diameter is 0.1 for all cylinder rotation speeds, this deviation from 0.2 can be attributed to the larger thermal boundary layer around the large cylinder (large slenderness ratio) at this Rayleigh number.

The corresponding maximum heat transfer density rates for $10^{2} \leq R a \leq 10^{4}$, $\operatorname{Pr}=0.72$ and $0 \leq \tilde{\omega}_{0} \leq 10$ are summarized in Fig. 6.12. From Fig. 6.12 it can be seen that any cylinder rotation in the range $0 \leq \tilde{\omega}_{0} \leq 1$ has almost no improvement on the the maximum heat transfer density rate for a multi scale structure. There is a maximum increase in heat transfer density rate of $1.2 \%, 1.5 \%$ and $1.2 \%$ for the $R a$ numbers $10^{2}, 10^{3}$ and $10^{4}$ respectively, when the cylinder rotation speed is increased from stationary to $\tilde{\omega}_{0}=1$. It is interesting to note that, at a cylinder rotation speed of $\tilde{\omega}_{0}=10$, the maximum heat transfer density rate is suppressed for all Rayleigh numbers in the range $10^{2} \leq R a \leq 10^{3}$.

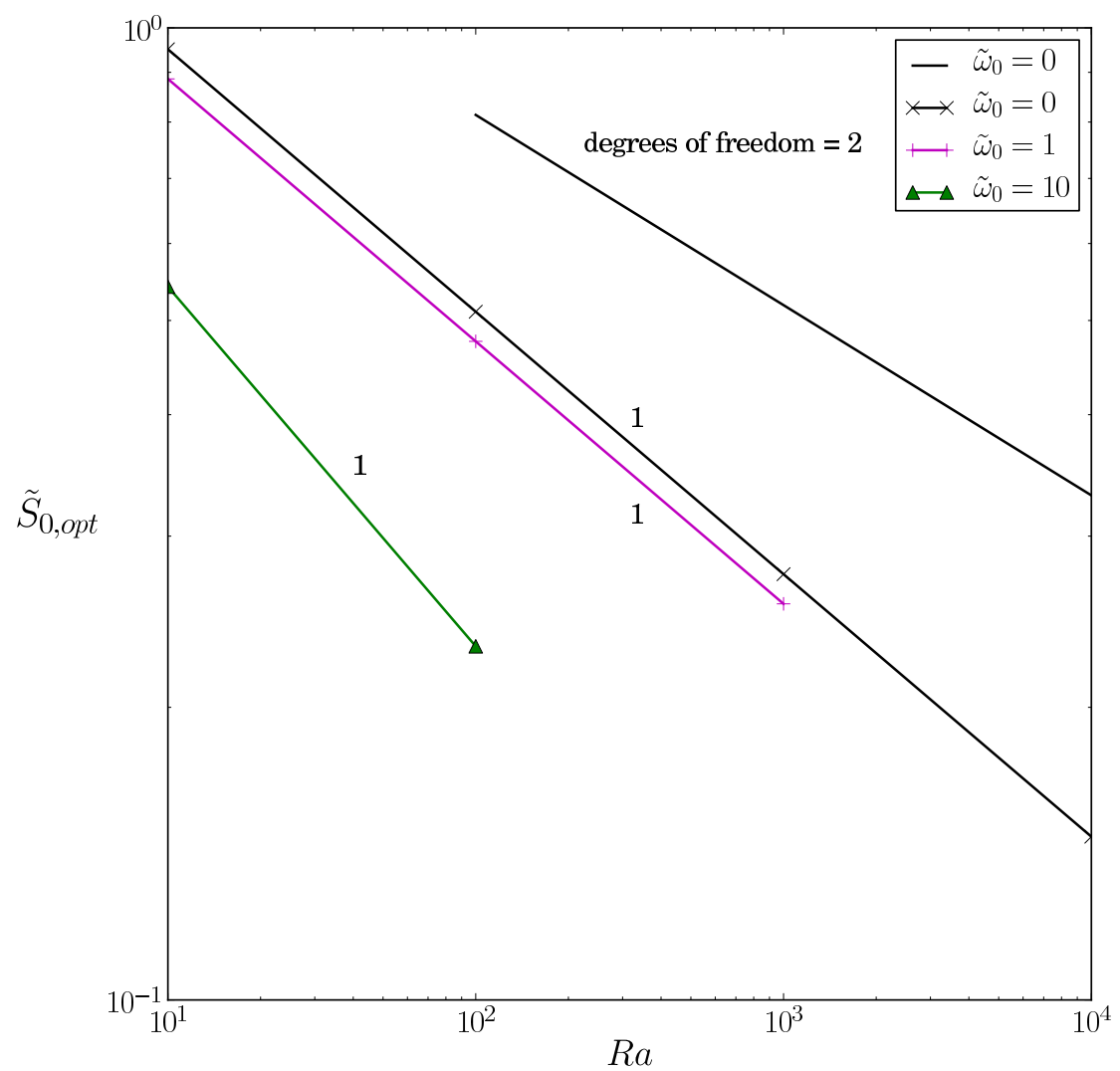

Figure 6.13: The effect of increasing of cylinder rotational speed and the effect of increasing the complexity on the optimal cylinder-to-cylinder spacing for $\operatorname{Pr}=0.72$. 
Figure 6.13 shows a comparison between the multi scale and single scale configurations for the optimal cylinder-to-cylinder. The multi scale configuration has the effect of increases the optimal cylinder-to-cylinder when adding a smaller diameter cylinder in the mouth of the channel. This smaller diameter cylinder also has the effect of restricting the flow through the mouth of the large diameter cylinders and thus cylinder rotation has a minimal effect on the cylinder-to-cylinder spacing. The maximum cylinder packing, from a physical point of view, is obtained using a single scale configuration with rotation.

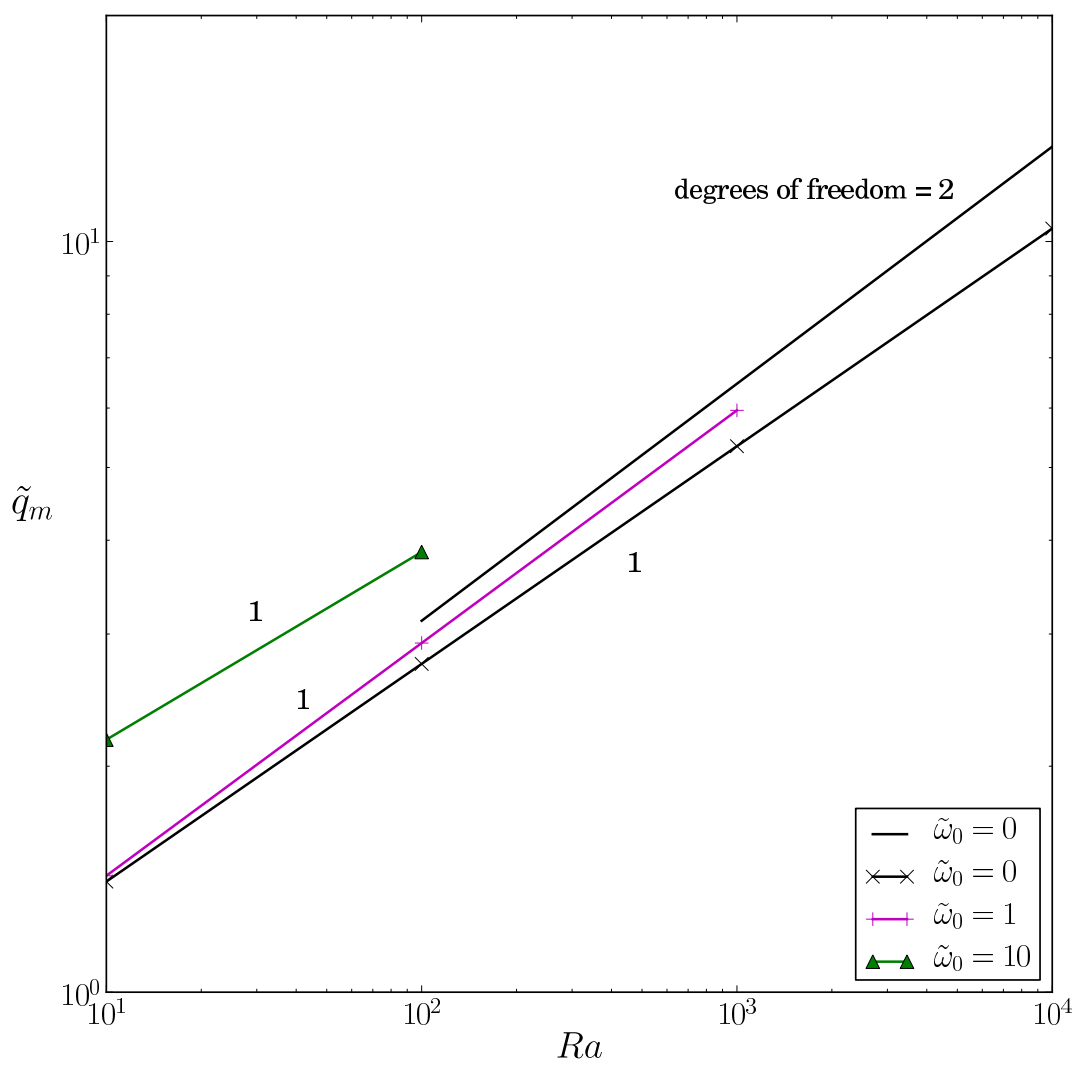

Figure 6.14: The effect of increasing of cylinder rotational speed and the effect of increasing the complexity on the maximum heat transfer density rate for $\operatorname{Pr}=0.72$.

Figure 6.14 shows a comparison between the multi scale and single scale configurations for the maximum heat transfer density rate. Although the effect of cylinder rotation (on a single scale configuration) is an increase in the maximum heat transfer density rate, a multi scale configuration (without any cylinder rotation) achieves a higher maximum heat transfer density rate. There is however an exception at very 
low Rayleigh numbers $\left(R a=10^{1}\right.$ and $\left.R a=10^{2}\right)$ where a high cylinder rotation speed increases the maximum heat transfer density rate greater than that of a multi scale configuration.

The optimal packing (both single scale and multi scale configurations) for the maximum heat transfer density rate for a given Rayleigh number and cylinder rotation speed can be presented in the following equality equation:

$$
\begin{aligned}
\text { if } 10^{1 \leq R a \leq 10^{2}:} & \\
\text { d.o.f. }= & 1 \\
\tilde{\omega}_{0}= & 10 \\
\tilde{S}_{0, \text { opt }}= & -0.362+1.69 R a^{-0.25} \\
& (\text { cf. Eq. } 6.7) \\
\tilde{q}_{m}= & 0.989+0.71 R a^{0.29} \\
& (\text { cf. Eq. } 6.8) \\
\text { if } 10^{2}<R a \leq 10^{4}: & \\
\text { d.o.f. }= & 2 \\
\tilde{\omega}_{0}= & 0 \\
\tilde{S}_{0, o p t}= & 2.113 R a^{(-0.205)} \\
& (\text { cf. Eq. } 6.9) \\
\tilde{q}_{m}= & 0.698 R a^{0.321} \\
& (\text { cf. Eq. 6.10) }
\end{aligned}
$$

where d.o.f. is the number of degrees of freedom for the flow configuration.

Figure 6.15 shows the effect of cylinder rotational speed on the thermal boundary layer for a multi scale structure of rotating cylinders shown in Fig. 5.3 for $R a=10^{3}$ and $\operatorname{Pr}=0.72$. Figure $6.15 \mathrm{a}$ shows, for $\tilde{\omega}_{0}=0$, that the thermal boundary layer between two consecutive large diameter cylinders, at the optimal geometric configuration, touches just past the top of the cylinders. Figure $6.15 \mathrm{~b}$ shows, for $\tilde{\omega}_{0}=1$, that the thermal boundary layer between two consecutive large diameter cylinders (where the cylinder rotation aids the flow direction) is extended and touches around one large cylinder diameter past the top of the cylinders. However, unlike the single scale configuration (cf. Fig. 6.7), the thermal boundary layer between two consecutive large 


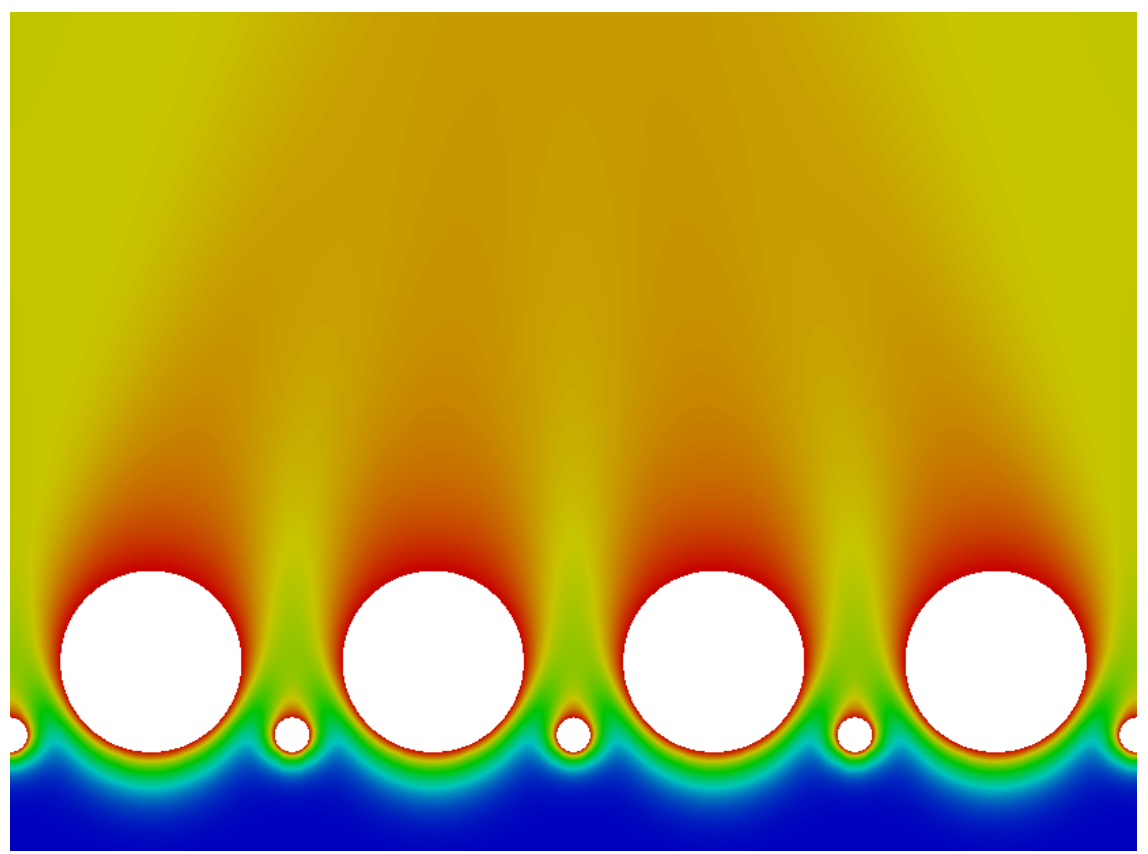

(a) $\tilde{\omega}_{0}=0$

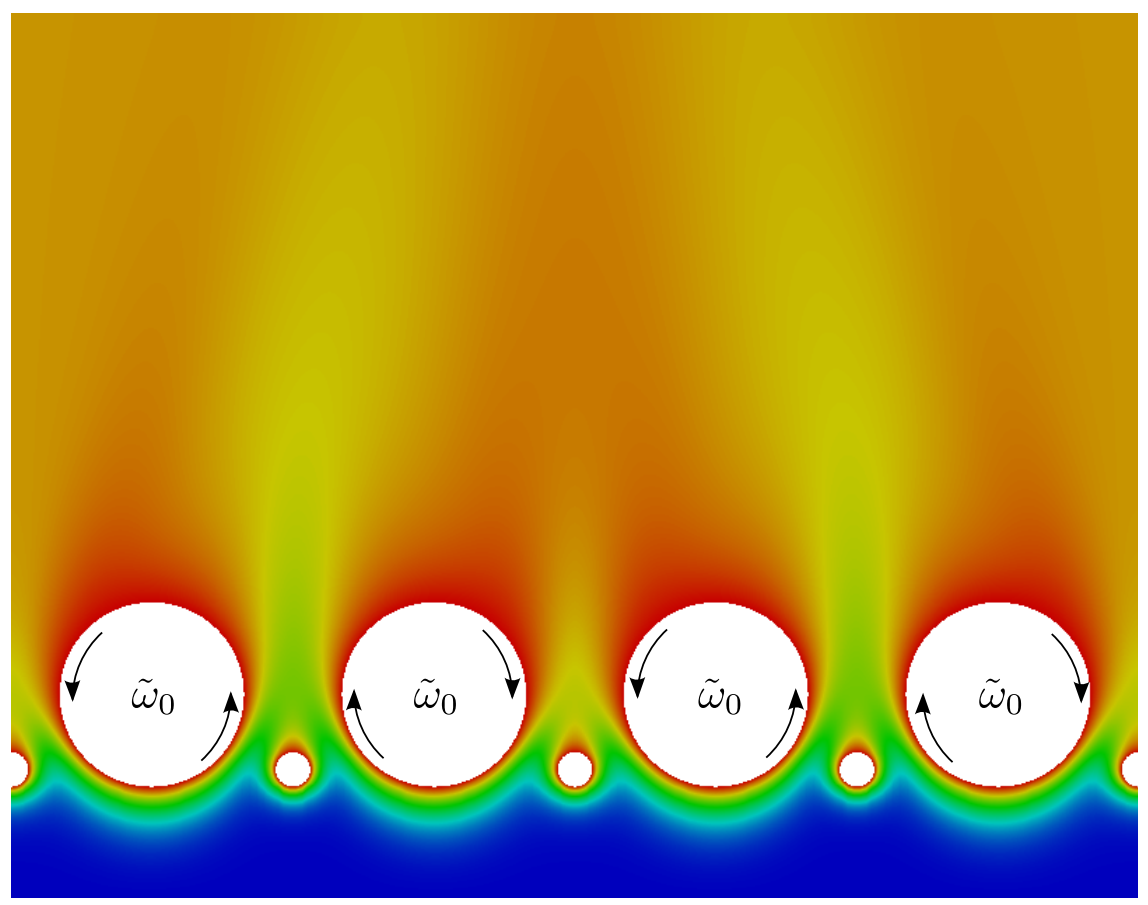

(b) $\tilde{\omega}_{0}=1$

Figure 6.15: The effect of cylinder rotational speed on the thermal boundary layer for a multi scale structure of rotating cylinders shown in Fig. 5.3 
diameter cylinders (where the cylinder rotation opposes the flow direction) is more strongly effected and touches near the centreline of the large diameter cylinders.

\subsubsection{Observations}

No definitive values for computational time and storage requirements were captured during the simulations of the computational domain. The computational time required to solve the fluid flow and temperature fields for the computational domain varied drastically (anywhere from a few minutes to a few days), depending on the number of elements in the mesh, the Rayleigh number, and cylinder rotational speed. All simulations were run using the same resources under the same conditions and thus the following trends were observed:

1. As the Rayleigh number was increased so was the number of elements in the computational domain (in order to achieve accuracy), which lead to an increase in storage requirements and computation time.

2. A higher Rayleigh number (leading to higher velocities) and a higher number of elements also lead to a smaller time step required to obtain a stable convergence and solution $\left(C_{o}=u \Delta t / \Delta x \leq 1\right)$. This smaller time step also leads to an increase in computational time.

3. The increase of the cylinder rotational speed had no effect on storage requirements but did slightly increase the computational time, although not to the extent of 1 and 2 above.

The time required for an optimisation run also varied drastically (anywhere from a few hours up to a week or two), again depending on the number of elements in the mesh, the Rayleigh number, and cylinder rotational speed.

\subsection{Conclusion}

This Chapter presented the results obtained for the optimal geometric configurations for the maximum heat transfer density rate, from the numerical models presented in 
Chapter 5. The results show that the addition of rotation to the cylinders serves to maximize the packing (minimize the cylinder-to-cylinder spacing and achieve a higher heat transfer density rate) of heat generating cylinders for a single scale configuration. Results further show that the addition of rotation to the cylinders, for a multi scale configuration, has almost no impact on improving the maximum packing. 


\section{Chapter 7}

\section{Summary, conclusions and recommendations}

\subsection{Summary}

In this paper we showed numerically the effect of counter-rotation on a row of heatgenerating cylinders which were cooled by natural convection. The cylinder-to-cylinder spacing was optimized for each flow regime and rotational speed on the cylinders. In the Rayleigh number range considered it was shown that the maximum heat transfer density rate increased and the optimal cylinder-to-cylinder spacing decreased with an increase in cylinder rotation speed for a single scale configuration at each Rayleigh number. For a multi scale configuration, the effect of increasing the rotation of the large diameter cylinders has little to no impact on the heat transfer density rate with the exception of a high rotation speed which serves to suppress the heat transfer density rate.

\subsection{Conclusions}

Cylinder rotation is beneficial for a single scale geometric design and serves to maximise the packing of the heat generating cylinders. For higher heat transfer density rates a 
multi scale geometric design becomes necessary, however the addition of rotation is no really beneficial. There are two effects of adding smaller cylinders in the wedge-shaped flow regions of the entrance to the assembly: 1) utilize the fluid that has not yet been used for heat transfer and 2) restrict the flow between the consecutive large cylinders. It is due to reason 2 that the optimal spacing is greater for a multi scale configuration than a single scale configuration.

It is also due to reason 2 that the effect of cylinder rotation has little impact on maximising the packing. As mentioned in Section 6.3.2.2 for cylinder rotation:

- The thermal boundary layer between two consecutive large diameter cylinders (where the cylinder rotation aids the flow direction) is extended and touches around one large cylinder diameter past the top of the cylinders: causing an increase in the heat transfer density rate.

- The thermal boundary layer between two consecutive large diameter cylinders (where the cylinder rotation opposes the flow direction) is strongly effected and touches near the centreline of the large diameter cylinders: causing a reduction in the heat transfer density rate; thereby cancelling each other out and yielding a net heat transfer density rate only slightly higher than the stationary cylinders.

\subsection{Recommendations for future work}

This section gives an overview of areas that have been identified as potential aspects for further research and investigation.

\subsubsection{Cylinder spacings and configurations}

The work presented in this thesis used only one spacing between consecutive large cylinders and only cylinder arrangement for the multi scale configuration. Future work may include extending the work in this thesis by considering multiple cylinder-to-cylinder spacings (as shown in Fig. 7.1) were it is expected that the cylinder spacing $S_{A}$ (where the flow is aided by the cylinder rotation) will be smaller than the cylinder 
spacing $S_{B}$. It may be worth investigating the effect of multiple cylinder-to-cylinder spacings on the heat transfer density rate as well as how $\left(S_{A}+S_{B}\right) / 2$ relates to $S_{0}$ presented in this thesis.

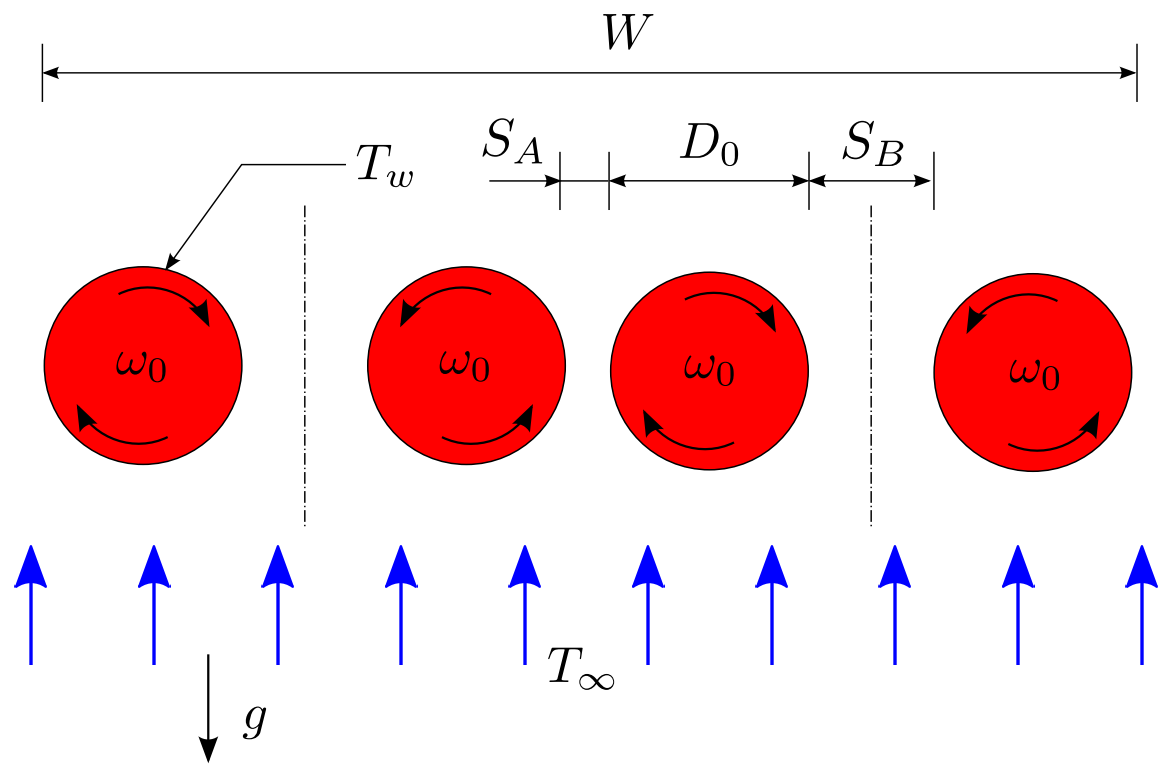

Figure 7.1: Single row of counter-rotating cylinders in natural convection with multiple cylinder-to-cylinder spacings.

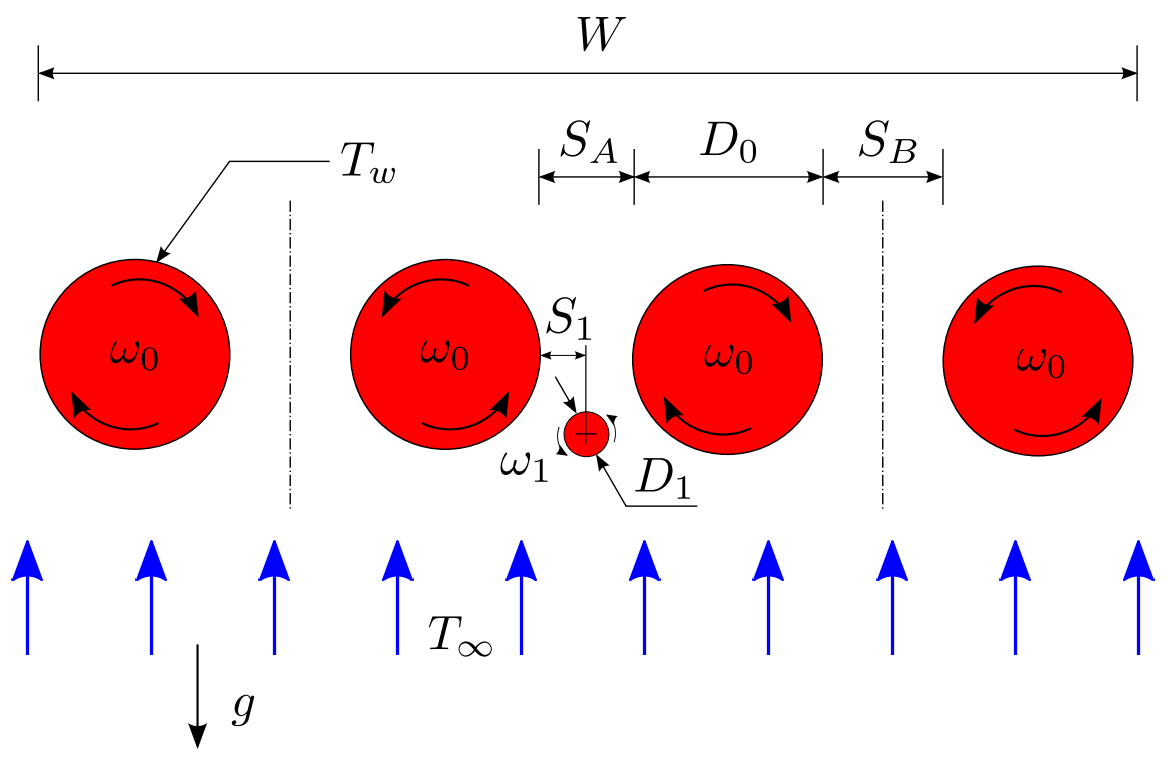

Figure 7.2: Multi scale row of counter-rotating cylinders in natural convection with multiple cylinder-to-cylinder spacings.

Further research may include considering various cylinder arrangements for the multi scale configuration (one example shown in Fig. 7.2). Again it may be worth 
investigating the effect of multiple cylinder-to-cylinder spacings on the heat transfer density rate. It may also be worth investigating the effect of small cylinder rotation and position (vertical and horizontal) on the heat transfer density rate. The multi scale configuration, for example, may be one with a staggered small cylinder arrangement (placed only by the large cylinder spacings where rotation aids the flow) or a uniform small cylinder arrangement (placed by all large cylinder spacings).

\subsubsection{Optimisation algorithm}

The optimisation algorithm used in this thesis ("fmin_slsqp") required a lot of objective function evaluations in order to determine the optimal geometric structure. Each objective function evaluation is in effect the solution for one CFD simulation for the given design variable values. A higher number of objective function evaluation leads to more time spent solving for the optimal structure, with the majority of the time being spent solving the CFD simulation. It would therefore be beneficial to research another optimisation algorithm or even develop a new algorithm, specifically tailored for CFD applications, that requires fewer objective function evaluation. Two suggested starting points would be with the work given in Refs [58, 63].

\subsubsection{Enhanced natural convection systems}

Future work in natural convection may include the complete design of a three dimensional pin fin heat sink (including physical side effects or boundary conditions) with the effect of pin rotation. Another possible research area for an enhanced natural convection system could be to consider the effect of vibration or oscillation of the pin fin on the heat transfer density rate.

Another possible research area could be to consider a tube type pin fin, in place of a solid volume pin fin, with perforation in the tube; thus allowing the fluid to move through the pin fin itself, allowing the flow configuration a lot more freedom to morph. 


\section{Publications in journals and conferences}

\section{Articles published}

1. L.G. Page, T. Bello-Ochende, J.P. Meyer, 'Maximum heat transfer density rate enhancement from cylinders rotating in natural convection', International Communications in Heat and Mass Transfer, 38:1354-1359, 2011. 


\section{References}

[1] GTO Media Inc. Hardware canucks, March 2008. URL http: //www . hardwarecanucks .com/forum/hardware-canucks-reviews/ 7054-asus-rampage-formula-x48-motherboard-review-5.html. [Online; accessed 10-March-2010].

[2] G.E. Moore. Cramming more components onto integrated circuits. Proceedings of the IEEE, 86(1):82 - 85, 1998.

[3] Wikipedia. Moore's law — wikipedia, the free encyclopedia, 2011. URL http://en.wikipedia.org/wiki/File:Transistor_Count_and_Moore\%27s_ Law_-_2011.svg. [Online; accessed 04-Jan-2012].

[4] J.R. Welty, C.E. Wicks, R.E. Wilson, and G. Rorrer. Fundamentals of Momentum, Heat, and Mass Transfer. John Wiley \& Sons, Inc., 4th edition, 2001.

[5] A. Bejan and S. Lorente. The constructal law and the thermodynamics of flow systems with configuration. International Journal of Heat and Mass Transfer, 47: $3203-3214,2004$.

[6] A. Bejan and S. Lorente. Design with Constructal Theory. John Wiley \& Sons, Inc., 1st edition, 2008.

[7] A. Bejan. Shape and structure, from engineering to nature. Technical report, Cambridge University Press, Cambridge, UK, 2000.

[8] A. Bejan, I. Dincer, S. Lorente, A.F. Miguel, and A.H. Reis. Porous and Complex Flow Structures in Modern Technologies. Springer-Verlag, New York, 1st edition, 2004. 
[9] A. Bejan and E. Sciubba. The optimal spacing for parallel plates cooled by forced convection. International Journal of Heat and Mass Transfer, 35:3259 - 3264, 1992.

[10] A. Bejan and A.M. Morega. The optimal spacing of a stack of plates cooled by turbulent forced convection. International Journal of Heat and Mass Transfer, 37: 1045 - 1048, 1994.

[11] A.K. da Silva, A. Bejan, and S. Lorente. Maximal heat transfer density in vertical morphing channels with natural convection. Numerical Heat Transfer Part A Applications, 45:135 - 152, 2004.

[12] T. Bello-Ochende and A. Bejan. Optimal spacings for mixed convection. Journal of Heat Transfer, 126:956 - 962, 2004.

[13] A. Bar-Cohen and W. M. Rohsenow. Thermally optimum spacing of vertical, natural convection cooled, parallel plates. ASME Journal of Heat Transfer, 106: $116-123,1984$.

[14] A. Bejan. Convection Heat Transfer. John Wiley \& Sons, Inc., 3rd edition, 2004.

[15] G. Stanescu, A.J. Fowler, and A. Bejan. The optimal spacing of cylinders in free-stream cross-flow forced convection. International Journal of Heat and Mass Transfer, 39:311 - 317, 1996.

[16] G. Stanescu, A.J. Fowler, and A. Bejan. The optimal spacing between horizontal cylinders in a fixed volume cooled by natural convection. International Journal of Heat and Mass Transfer, 38:2047 - 2055, 1995.

[17] T. Bello-Ochende and A. Bejan. Maximal heat transfer density: plates with multiple lengths in forced convection. International Journal of Thermal Sciences, 43: $1181-1186,2004$.

[18] A. Bejan and Y. Fautrelle. Constructal multi-scale structure for maximal heat transfer density. Acta Mechanica, 163:39 - 49, 2003.

[19] T. Bello-Ochende, J.P. Meyer, and J. Dirker. Three-dimensional multi-scale plate assembly for maximum heat transfer rate density. International Journal of Heat and Mass Transfer, 53:586 - 593, 2010. 
[20] A.K. da Silva and A. Bejan. Constuctal multi-scale structure for maximal heat transfer density in natural convection. International Journal of Heat and Fluid Flow, 26:34 - 44, 2005.

[21] T. Bello-Ochende and A. Bejan. Constructal multi-scale cylinders in cross-flow. International Journal of Heat and Mass Transfer, 48:1373 - 1383, 2005.

[22] T. Bello-Ochende and A. Bejan. Constructal multi-scale cylinders with natural convection. International Journal of Heat and Mass Transfer, 48:4300 - 4306, 2005.

[23] H.M. Badr and S.C.R. Dennis. Laminar forced convection from a rotating cylinder. International Journal of Heat and Mass Transfer, 28:253 - 264, 1985.

[24] C.C. Chiou and S.L. Lee. Forced convection on a rotating cylinder with an incident air jet. International Journal of Heat and Mass Transfer, 36:3841 - 3850, 1993.

[25] S.K. Panda and R.P. Chhabra. Laminar forced convection heat transfer from a rotating cylinder to power-law fluids. Numerical Heat Transfer; Part A: Applications, 59:297 - 319, 2011.

[26] M.A. Gshwendtner. Optical investigation of the heat transfer from a rotating cylinder in a cross flow. Heat and Mass Transfer, 40:561 - 572, 2004.

[27] A.K. Mohanty, A.A. Tawfek, and B.V.S.S.S. Prasad. Heat transfer from a rotating cylinder in crossflow. Experimental Thermal and Fluid Science, 10:54 - 61, 1995.

[28] M. Oesterle, M. Lauster, R. Waibel, V. Lippig, and D. Straub. Topological structures near a heated rotating cylinders. Experiments in Fluids, 24:308 - 322, 1998.

[29] B. Ozerdem. Measurement of convective heat transfer coefficient for a horizontal cylinder rotating in quiescent air. International Communications in Heat and Mass Transfer, 27:389 - 395, 2000.

[30] S.B. Paramane and A. Sharma. Numerical investigation of heat and fluid flow across a rotating circular cylinder maintained at constant temperature in 2-d laminar flow regime. International Journal of Heat and Mass Transfer, 52:3205 - 3216, 2009.

[31] S.B. Paramane and A. Sharma. Heat and fluid flow across a rotating cylinder dissipating uniform heat flux in $2 \mathrm{~d}$ laminar flow regime. International Journal of Heat and Mass Transfer, 53:4672 - 4683, 2010. 
[32] Y.Y. Yan and Y.Q. Zu. Numerical simulation of heat transfer and fluid flow past a rotating isothermal cylinder - a lbm approach. International Journal of Heat and Mass Transfer, 51:2519 - 2536, 2008.

[33] M.R.H. Nobari, K. Gharali, and M. Tajdari. A numerical study of flow and heat transfer in internally finned rotating curved pipes. Numerical Heat Transfer; Part A: Applications, 56:76 - 95, 2009.

[34] M. Joucaviel, L. Gosselin, and T. Bello-Ochende. Maximum heat transfer density with rotating cylinders aligned in cross-flow. International Communications in Heat and Mass Transfer, 35:557 - 564, 2008.

[35] T. Bello-Ochende, J.P. Meyer, and O.I. Ogunronbi. Constructal multiscale cylinders rotating in cross-flow. International Journal of Heat and Mass Transfer, 54: $2568-2577,2011$.

[36] H.K. Versteeg and W. Malalasekera. An introduction to computational fluid dynamics: the finite volume method. Prentice Hall, England, 2nd edition, 2007.

[37] OpenCFD Ltd. OpenFOAM: The open source cfd toolbox, August 2011. URL http://www.openfoam.com. [Online; accessed 11-October-2011].

[38] OpenCFD Ltd. OpenFOAM: User guide, August 2011. URL http://www. openfoam.com/docs/user/. [Online; accessed 11-October-2011].

[39] J.W. Eaton. Octave, October 2011. URL http://www.gnu.org/software/ octave/. [Online; accessed 19-October-2011].

[40] Python Software Foundation. Python programming language - official website, September 2011. URL http://www.python.org/. [Online; accessed 19-October2011].

[41] F.M. White. Viscous Fluid Flow. McGraw-Hill International Editions, Singapore, 3rd edition, 2006.

[42] A. Bejan and A.D. Kraus. Heat Transfer Handbook. John Wiley \& Sons, Inc., 2003.

[43] H. Jasak. Error Analysis and Estimation for the Finite Volume Method with Applications to Fluid Flows. PhD thesis, Department of Mechanical Engineering Imperial College of Science, Technology and Medicine, 1996. 
[44] C.M. Rhie and W.L. Chow. A numerical study of the turbulent flow past as isolated airfoil with trailing edge separation. In AIAA-82-0998, AIAA/ASME 3rd Joint Thermophysics, Fluids, Plasma and Heat Transfer Conference, St. Louis, Missouri, 1982.

[45] M. Perić. A Finite Volume method for the prediction of three-dimensional fluid flow in complex ducts. PhD thesis, Imperial College, University of London, 1985.

[46] J.H. Ferziger and M. Perić. Computational methods for fluid dynamics. Springer, 3rd edition, 2002.

[47] S.V Patankar and D.B Spalding. A calculation procedure for heat, mass and momentum transfer in three-dimensional parabolic flows. International Journal of Heat and Mass Transfer, 15(10):1787 - 1806, 1972.

[48] J.P. Van Doormaal and G.D. Raithby. An evaluation of the segregated approach for predicting incompressible fluid flows. In In National Heat Transfer Conference, Denver, Colorado, August 1985.

[49] F.P. Kärrholm. Numerical Modelling of Diesel Spray Injection, Turbulence Interaction and Combustion. PhD thesis, Department of Applied Mechanics Chalmers, University of Technology Goteborg, Sweden, 2008.

[50] R.I. Issa. Solution of the implicitly discretised fluid flow equations by operatorsplitting. Journal of Computational Physics, 62(1):40 - 65, 1986.

[51] A.K. da Silva and L. Gosselin. Volumetric maximization of coolant usage in closed self-driven circuits. International Journal of Heat and Mass Transfer, 53(19-20): 3969 - 3976, 2010.

[52] T. Williams, C. Kelley, and Others. Gnuplot homepage, March 2011. URL http: //www.gnuplot.info/. [Online; accessed 14-November-2011].

[53] E. Jones, T. Oliphant, P Peterson, and Others. SciPy: Open source scientific tools for Python, November 2011. URL http://www. scipy.org/. [Online; accessed 14November-2011].

[54] B Gschaider. Contrib PyFoam, November 2011. URL http://openfoamwiki. net/index.php/Contrib_PyFoam. [Online; accessed 5-December-2011]. 
[55] J.A. Snyman. Practical mathematical optimisation: an introduction to basic optimisation theory and classical and new gradient-based algorithms. Springer, New York, 2005.

[56] T. Bello-Ochende, J.P. Meyer, and F.U. Ighalo. Combined numerical optimization and constructal theory for the design of microchannel heat sinks. Numerical Heat Transfer, Application Part A, 58:882 - 899, 2010.

[57] F.U. Ighalo. Optimisation of microchannels and micropin-fin heat sinks with computational fluid dynamics in combination with a mathematical optimisation algorithm. Master's thesis, Faculty of Engineering, Built Environment and Information Technology, University of Pretoria, 2010.

[58] J.A. Snyman and Hay A.M. The dynamic-q optimisation method: an alternative to sqp? Computer and Mathematics with Applications, 44:1589 - 1598, 2002.

[59] J.A. Snyman. A new and dynamic method for unconstrained minimization. Applied. Mathematical Modelling, 6:449 - 462, 1982.

[60] J.A. Snyman. An improved version of the original leap-frog dynamic method for unconstrained minimization: LFOP1(b). Applied. Mathematical Modelling, 7:216 $218,1983$.

[61] J.A. Snyman, N. Stander, and W.J. Roux. A dynamic penalty function method for the solution of structural optimisation problems. Applied. Mathematical Modelling, 18:453 - 460, 1994.

[62] J.A. Snyman. The lfopc leap-frog algorithm for constrained optimisation. Computer and Mathematics with Applications, 40:1085 - 1096, 2000.

[63] D.N. Wilke, S. Kok, and A.A. Groenwold. The application of gradient-only optimization methods for problems discretized using non-constant methods. Structural and Multidisciplinary Optimization, 40:433 - 451, 2010.

[64] D. Kraft. Algorithm 733: Tomp - 8211; fortran modules for optimal control calculations. ACM Trans. Math. Softw., 20:262-281, 1994.

[65] C.L. Lawson and Hanson R.J. Solving Least Square Problems. Prentice-Hall, Englewood Cliffs, New Jersey, 1974. 


\section{Appendix A: Nomenclature}

\section{Latin symbols}

A Area

$m^{2}$

$a_{P} \quad$ Central coefficient

$a_{n b} \quad$ Coefficient corresponding to neighbour element

$\mathcal{A} \quad$ Central coefficient matrix

$\stackrel{A}{=} \quad$ Coefficient matrix

$b \quad$ Source term matrix

D Diffusion conductance coefficient

F $\quad$ Convective mass flux coefficient

$\mathrm{kg} / \mathrm{s}$

$\mathcal{H} \quad$ Transport and source coefficient matrix

$n \quad$ Surface normal vector

$P \quad$ Pressure

$P a$

$P_{d} \quad$ Dynamic pressure $\quad P a$

$S \quad$ Surface $m^{2}$

$S_{u} \quad$ Constant part of the conservation equation source term -

$S_{P} \phi \quad$ Linear part of the conservation equation source term

$S_{\phi} \quad$ Conservation equation source term 
$t \quad$ Time / Time step

T Fluid temperature $\quad K$

$T_{\infty} \quad$ Inlet fluid temperature $\quad K$

U Velocity vector $\mathrm{m} / \mathrm{s}$

$u, v, w \quad$ Velocity components $\mathrm{m} / \mathrm{s}$

$V \quad$ Element volume $m^{3}$

$x, y, z \quad$ Cartesian coordinates $m$

\section{Greek symbols}

$\alpha \quad$ Thermal diffusivity $\mathrm{m}^{2} / \mathrm{s}$

$\alpha_{f} \quad$ Mathematical averaging function

$\beta \quad$ Thermal expansion coefficient $1 / K$

$\Gamma_{\phi} \quad$ Diffusion coefficient

$\delta_{A P} \quad$ Distance between the centroids of elements A and P $m$

$\delta_{A f} \quad$ Distance between the centroid and face of elements A $m$

$\delta_{f P} \quad$ Distance between the face and centroid of elements P $m$

$\mu \quad$ Viscosity $\mathrm{kg} / \mathrm{ms}$

$\rho \quad$ Fluid density $\quad \mathrm{kg} / \mathrm{m}^{3}$

$\rho_{o} \quad$ Fluid reference density $\mathrm{kg} / \mathrm{m}^{3}$

$\phi \quad$ Scalar variable

\section{Subscripts}

A Arbitrary control volume adjacent to element $\mathrm{P}$

$e \quad$ East face of element $\mathrm{P}$

E Centre of the control volume east of element P 
$f \quad$ Values at the face centre of the control volume

$n b \quad$ Neighbour element

$P \quad$ Values at the centre of the control volume

$t \quad$ Time step

$w \quad$ West face of element $\mathrm{P}$

$W \quad$ Centre of the control volume west of element $\mathrm{P}$

\section{Accents}

Finite number of selected points

$-\quad$ Weighted linear interpolation 


\section{Appendix A}

\section{Discretisation methods}

\section{A.1 Discretisation of the momentum equation}

The work presented in this section and sub-sections includes a summary of the work presented in Chapter 2 of Croft [A1]. Consider the general form of a given conservation equation, for a scalar variable $\phi$ :

$$
\left.\underset{\text { Transient }}{\frac{\partial(\rho \phi)}{\partial t}}+\underset{\text { Convection }}{\operatorname{div}(\rho \underline{u} \phi)}=\operatorname{div} \underset{\text { Diffusion }}{\left[\Gamma_{\phi} \operatorname{grad}\right.}(\phi)\right]+\underset{\text { Source }}{S_{\phi}}
$$

where $\phi$ is a scalar variable, $\Gamma_{\phi}$ is the diffusion coefficient and $S_{\phi}$ is the $\phi$ source term.

Equation A.1 can be written in vector form, to represent the governing momentum equation (Eq. 3.8), as:

$$
\frac{\partial(\rho \mathbf{U})}{\partial t}+\operatorname{div}(\rho \underline{\mathbf{U}} \mathbf{U})=\operatorname{div}[\mu \operatorname{grad}(\mathbf{U})] \underbrace{-\nabla P_{d}-\rho_{0} \mathbf{g} \beta\left(T-T_{\infty}\right)}_{\text {Source }}
$$

Equation A.1 can also be written in vector form, to represent the governing energy equation (Eq. 3.11), as:

$$
\frac{\partial(T)}{\partial t}+\operatorname{div}(\underline{\mathbf{U}} T)=\operatorname{div}[\alpha \operatorname{grad}(T)]+\underbrace{0}_{\text {Source }}
$$


The aim of discretising Eq. A.1 is to obtain suitable approximations for each term in Eq. A.1 such that it can be expressed in a linear matrix equation of the form:

$$
\underline{A} \underline{\underline{\phi}}=\underline{b}
$$

where $\phi$ is a vector of the values of $\phi$ at a finite number of selected points, such that the solution of Eq. A.4 gives as close an approximation as possible to the solution of Eq. A.1 [A1]. The method used to discretise Eq. A.1 is the Finite Volume Method (FVM) presented in Section 3.5.1 and all terms in Eq. A.1 are discretised from the integral form over each control volume.

\section{A.1.1 Transient term}

The transient term, integrated over time and a control volume, is:

$$
\int_{t-\Delta t}^{t} \int_{V} \frac{\partial(\rho \phi)}{\partial t} \mathrm{~d} V \mathrm{~d} t
$$

If the mesh is stationary, the control volume $V$ does not change with time, then Eq. A.5 becomes:

$$
\int_{V}\left(\rho^{i} \phi^{i}-\rho^{i-1} \phi^{i-1}\right) \mathrm{d} V
$$

where $i$ denotes the time step. Assuming that the values at the centre of the control volume, signified by the subscript $P$, are representative of the average value in the control volume then Eq. A.6 can be approximated by

$$
V_{P}\left(\rho_{P}^{i} \phi_{P}^{i}-\rho_{P}^{i-1} \phi_{P}^{i-1}\right)
$$

where $V_{P}$ is the volume of the control volume.

For every other term in the momentum equation fully implicit assumptions are used. This means that the time integration of the remaining terms simply leads to a multiplying factor of $\Delta t$. The whole discretised equation is divided by this multiplying factor so that the choice of time step only affects the contribution from the transient term. 
Thus the final form of the discretised transient term is

$$
\frac{V_{P}\left(\rho_{P}^{i} \phi_{P}^{i}-\rho_{P}^{i-1} \phi_{P}^{i-1}\right)}{\Delta t}
$$

The integration over time will thus be ignored in the following sections that describe the discretisation of the remaining terms [A1].

\section{A.1.2 Convection term}

The first step in the discretisation of the convection term is to use the divergence theorem:

$$
\int_{V} \operatorname{div}(\underline{F}) \mathrm{d} V=\int_{S}(\underline{F} \cdot \underline{n}) \mathrm{d} S
$$

where $\underline{n}$ is the outward unit vector normal to the surface and $\mathbf{F}$ is a continuous differentiable vector field defined on a neighbourhood of $V$. The divergence theorem (Eq. A.9) is used to convert the convection term from a volume integral to a surface integral:

$$
\int_{V} \operatorname{div}(\rho \underline{u} \phi) \mathrm{d} V=\int_{S} \rho(\underline{u} \cdot \underline{n}) \phi \mathrm{d} S
$$

The surface integral is again split into a set of integrals over each of the faces bounding the control volume and each of the values in the integrand are estimated on the face to give:

$$
\sum_{f} \rho_{f}(\underline{u} \cdot \underline{n})_{f} \phi_{f} A_{f}
$$

In this equation the value of $\rho_{f}$ is given the value in the upwind element. Thus

$$
\begin{array}{ll}
\rho_{f}=\rho_{P} & \text { if }(\underline{u} \cdot \underline{n})_{f}>0.0 \\
\text { or } \quad & \text { if }(\underline{u} \cdot \underline{n})_{f}<0.0
\end{array}
$$

The Rhie - Chow interpolation method, used to evaluate the normal component of velocity at the face, $(\underline{u} \cdot \underline{n})_{f}$, will be described later in section A.1.6. To complete the discretisation of the convection term some approximation has to be made regarding the 
calculation of the face value of $\phi$. One possible method is to use arithmetic averaging:

$$
\begin{aligned}
\phi_{f} & =\alpha_{f} \phi_{P}+\left(1-\alpha_{f}\right) \phi_{A} \\
\alpha_{f} & =\frac{\delta_{A f}}{\delta_{A f}+\delta_{f P}}
\end{aligned}
$$

Assuming that arithmetic averaging is being used the discretised form of the convection term becomes:

$$
\sum_{f} \rho_{f}(\underline{u} \cdot \underline{n})_{f}\left[\alpha_{f} \phi_{P}+\left(1-\alpha_{f}\right) \phi_{A}\right] A_{f}
$$

\section{A.1.3 Diffusion term}

As with the convection term the divergence theorem (Eq. A.9) is used to transform the volume integral of the diffusion term into a surface integral:

$$
\int_{V} \operatorname{div}\left[\Gamma_{\phi} \operatorname{grad}(\phi)\right] \mathrm{d} V=\int_{S} \Gamma_{\phi} \operatorname{grad}(\phi) \cdot \underline{n} \mathrm{~d} S
$$

Equation A.15 can be simplified further by recognising that $\operatorname{grad}(\phi) \cdot \underline{n}$ is equal to the gradient of $\phi$ in the direction of the normal. The overall surface of any polyhedral control volume consists of a set of faces and thus Eq. A.15 can be written as a sum of surface integrals over each face bounding the control volume:

$$
\sum_{f} \int_{f} \Gamma_{\phi} \frac{\partial \phi}{\partial n} \mathrm{~d} S
$$

Consider an element $A$ adjacent to element $P$, such that the two elements share a face $f$. For simplicity it is assumed that the mesh is fully orthogonal. The normal gradient in Eq. A.16 is then approximated as:

$$
\frac{\partial \phi}{\partial n} \simeq \frac{\phi_{A}-\phi_{P}}{\delta_{A P}}
$$

where $\delta_{A P}$ is the distance between the centroids of elements $\mathrm{A}$ and $\mathrm{P}$. The discretised 
form of Eq. A.15 becomes:

$$
\sum_{f}\left(\Gamma_{\phi}\right)_{f}\left(\frac{\phi_{A}-\phi_{P}}{\delta_{A P}}\right) A_{f}
$$

where $A_{f}$ is the area of face $f$. The only remaining decision is how to calculate the coefficient $\Gamma_{\phi}$ on the face. This is often done by means of an arithmetic mean:

$$
\begin{aligned}
\left(\Gamma_{\phi}\right)_{f} & =\alpha_{f}\left(\Gamma_{\phi}\right)_{P}+\left(1-\alpha_{f}\right)\left(\Gamma_{\phi}\right)_{A} \\
\alpha_{f} & =\frac{\delta_{A f}}{\delta_{A f}+\delta_{f P}}
\end{aligned}
$$

\section{A.1.4 Source term}

When the source term is discretised it is initially expressed in a linearised form [36]:

$$
S_{\phi}=S_{u}-S_{P} \phi
$$

where $S_{u}$ and $S_{P}$ can be functions of any stored value including $\phi$. To maintain diagonal dominance of the resulting equation the value of $S_{P}$ must not be negative. The equations used to evaluate the values of $S_{u}$ and $S_{P}$ can significantly affect both the rate of convergence and stability of the solution procedure. For speed as large a portion of the source as possible should be placed in the linear, $S_{P} \phi$, part. This speed will be compromised if the size of $S_{u}$ is also increased. Stability will be affected if changes in any aspect of the solution results in large changes in the values of $S_{u}$ and $S_{P}$.

When the linearised source term is integrated over the control volume all terms are evaluated at the centre of the element to give a contribution:

$$
\left(S_{u}-S_{P} \phi_{P}\right) V_{P}
$$

The importance of the linearisation becomes clear in implicit calculations. It is advisable to treat the source term as "implicitly" as possible. 


\section{A.1.5 Convection and diffusion terms}

Having obtained expressions for the discretised form of each of the terms in the conservation equation in the previous section, the discretised form of the full equation is obtained by simply adding together all these contributions. If only the convection and diffusion terms are considered, and using arithmetic averaging in the evaluation of the face value of $\phi$ in the convection term, the discretised equation becomes:

$$
\sum_{f}\left[\rho_{f}(\underline{u} \cdot \underline{n})_{f}\left\{\alpha_{f} \phi_{P}+\left(1-\alpha_{f}\right) \phi_{A}\right\}+\left(\Gamma_{\phi}\right)_{f}\left(\frac{\phi_{A}-\phi_{P}}{\delta_{A P}}\right)\right] A_{f}=0
$$

The quantities $F_{f}$ and $D_{f}$ are now introduced where:

$$
F_{f}=\rho_{f}(\underline{u} \cdot \underline{n})_{f} A_{f} \quad D_{f}=\left(\Gamma_{\phi}\right)_{f} A_{f} / \delta_{A P}
$$

$F_{f}$ is the strength of the convection of $\phi$ and $D_{f}$ is the diffusion conductance. Now Eq. A.22 can be rewritten as:

$$
a_{P} \phi_{P}=\sum_{n b} a_{n b} \phi_{n b}
$$

where the summation is over all neighbouring elements. The equations for the coefficients in Eq. A.24 are:

$$
\begin{aligned}
a_{n b} & =D_{f}-\left(1-\alpha_{f}\right) F_{f} \\
a_{P} & =\sum_{f}\left(D_{f}+\alpha_{f} F_{f}\right) \\
& =\sum_{n b} a_{n b}+\sum_{f} F_{f}
\end{aligned}
$$

\section{A.1.6 Rhie - Chow interpolation method}

If the one dimensional $x$ momentum equation is considered:

$$
\frac{\partial(\rho u)}{\partial t}+\frac{\partial(\rho \underline{u} u)}{\partial x}=\frac{\partial}{\partial x}\left(\mu \frac{\partial u}{\partial x}\right)-\frac{\partial P}{\partial x}
$$


where $u$ is the $x$ component of velocity. The pressure derivative term must be discretised over the control volume. If the control volume considered contains the node $P$ with neighbouring nodes $W$ and $E$ (as shown in Fig. A.1) then the contribution to the discretised equation from this term is $P_{e}-P_{w}$. Assuming that the faces $e$ and $w$ are midway between the nodes either side of them, then using linear interpolation for the face values of pressure:

$$
P_{w}-P_{e}=\frac{P_{W}+P_{P}}{2}-\frac{P_{P}+P_{E}}{2}=\frac{P_{W}+P_{E}}{2}
$$

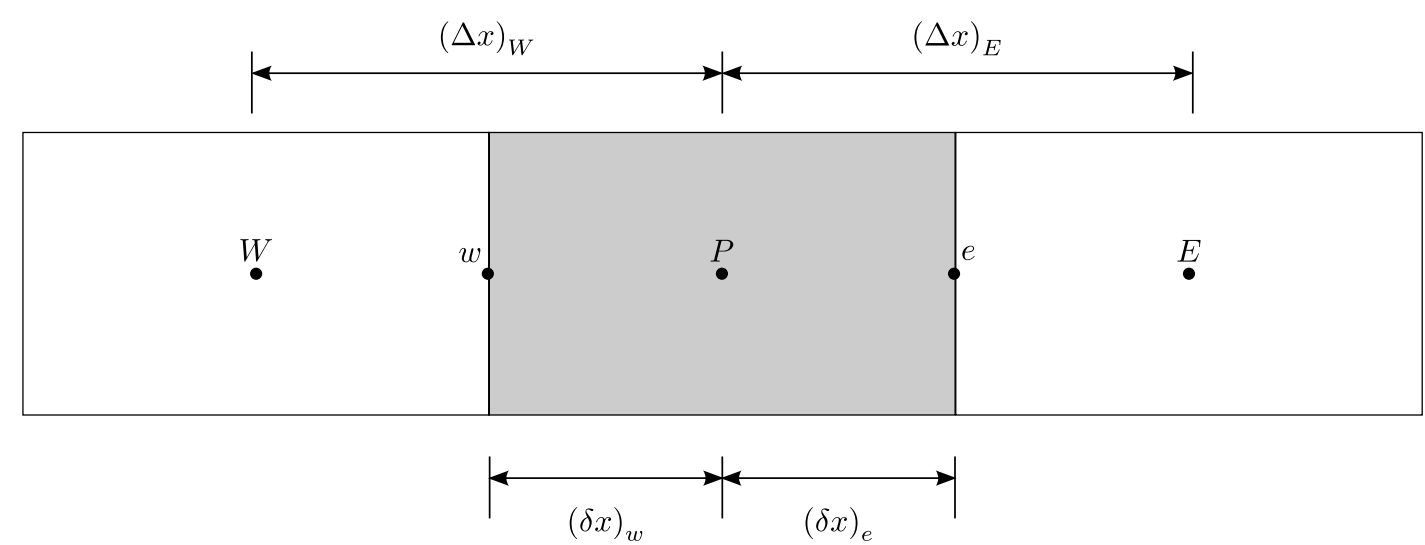

Figure A.1: One dimensional control volume.

This indicates that the pressure differential term $\left(\frac{\partial P}{\partial x}\right)$ in the momentum equation leads to a discretised form which is a relationship between alternate nodal pressure values rather than adjacent ones. As a consequence the pressure differential is calculated over a coarser mesh than the one used for all other quantities. It also means that an oscillatory pressure field, in which, for example, the pressure at consecutive grid points follows the sequence $[1,100,1,100,1$ etc.], would be treated as a uniform pressure field due to the alternate grid points having the same value.

In higher dimensions the problem leads to the checkerboard effect where in each of the dimensions alternate grid points have the same pressure value but not adjacent nodes. This means that in three dimensions the pressure field could take 8 different values but still the discretised pressure differential term would give a zero pressure differential.

Consider now the steady one dimensional continuity equation for an incompressible 
flow, given as:

$$
\frac{\partial u}{\partial x}=0
$$

Integrating this over the control volume of Fig. A.1, and assuming that the faces lie midway between their nodes, the following equation is obtained:

$$
\begin{array}{ll} 
& u_{e}-u_{w}=0 \\
\text { or } \quad & \frac{u_{P}+u_{E}}{2}-\frac{u_{W}+u_{P}}{2}=0 \\
\text { or } \quad u_{E}-u_{W}=0
\end{array}
$$

The problems of an equation which tests alternate nodal values, rather than adjacent ones, is again obtained. Hence, similar to the pressure, a checkerboard velocity field would satisfy the continuity equation.

The standard mechanism for handling the above problem is the use of a staggered mesh. The staggered grid for the velocity components was first suggested by Harlow and Welch [A2] and forms the basis of the SIMPLE procedure [47]. In a staggered grid the $u$ velocity is calculated at the faces of the control volume which have their normal vector in the $x$ coordinate direction. Similarly the $v$ and $w$ velocities are calculated at the faces of the control volume with normal vectors in the $y$ and $z$ directions respectively. The calculation of the velocities at the control volume faces means that in Eq. A.28 the values of $u_{e}$ and $u_{w}$ are obtained directly from the solution of the $u$ component of velocity. This means that the discretised continuity equation now consists of a relationship between adjacent $u$ values and so the checkerboard velocity pattern is no longer possible. Similarly the staggered grid leads to the pressure differential between adjacent nodes becoming the driving force of the velocity component solved for between these nodes.

This staggering of the velocity components is of huge benefit on structured meshes but the method does not extend easily to unstructured meshes. In the approach presented in this thesis, all quantities are solved and stored at the element centroid. The face values of the velocity components have to be calculated from these element based values. This leads to the need to employ an alternative interpolation method, to those described earlier in this section, which does not suffer from the checkerboard effect. 
The Rhie - Chow interpolation method [44] offers one approach which satisfies these requirements.

The $u$ momentum equation

$$
\frac{\partial(\rho u)}{\partial t}+\operatorname{div}(\rho \underline{u} u)=-\frac{\partial P}{\partial x}+\operatorname{div}[\mu \operatorname{grad}(u)]+S_{u}
$$

can be discretised, using the techniques described earlier in this chapter, over the control volume about a node $P$ to produce an equation which can be written in the form:

$$
a_{P} u_{P}+\left(\nabla_{x} P\right)_{P}=\left(\sum a_{n b} u_{n b}\right)_{P}+S_{u, P}
$$

where $\nabla_{x} p$ is the discretised contribution from the pressure gradient term. Similarly for the adjacent node $A$ :

$$
a_{A} u_{A}+\left(\nabla_{x} P\right)_{A}=\left(\sum a_{n b} u_{n b}\right)_{A}+S_{u, A}
$$

From the conservation principle of the control volume, the formulation of the $u$ velocity, at a point on the face between the nodes, must also have a discretised momentum equation of the form:

$$
a_{f} u_{f}+\left(\nabla_{x} P\right)_{f}=\left(\sum a_{n b} u_{n b}\right)_{f}+S_{u, f}
$$

The Rhie - Chow interpolation method uses Eq. A.31 and Eq. A.32 to approximate a solution of Eq. A.33. It is assumed that the right hand side of Eq. A.33 may be approximated by using a weighted linear interpolation of the corresponding terms in Eq. A.31 and Eq. A.32. Thus:

$$
\begin{aligned}
a_{f} u_{f}+\left(\nabla_{x} P\right)_{f} & =\overline{a_{f} u_{f}}+\overline{\left(\nabla_{x} P\right)_{f}} \\
& =\overline{\left(\sum a_{n b} u_{n b}\right)_{f}}+\overline{S_{u, f}}
\end{aligned}
$$

where the over-line in the above equation indicates a weighted linear interpolation of the variable. 


\section{A.1.6.1 Implementation in OpenFoam}

Consider the complete mass conservation equation (Eq. 3.2) for steady-state incompressible flow. Again the divergence theorem (Eq. A.9) is used to transform the volume integral of the continuity equation into a surface integral:

$$
\int_{V} \operatorname{div}(\mathbf{U}) \mathrm{d} V=\sum_{f}\left(\mathbf{U}_{f} \cdot \underline{n}\right) A_{f}=0
$$

The complete momentum equation (Eq. A.2) can also be discretised, using the techniques described earlier in this chapter, over the control volume about a node $P$ to produce an equation which can be written in the same form as Eq. A.34:

$$
\begin{aligned}
\mathcal{A}_{f} \mathbf{U}_{f}+(\nabla P)_{f} & =\overline{\mathcal{A}_{f} \mathbf{U}_{f}}+\overline{(\nabla P)_{f}} \\
& =\overline{\left(\sum \mathcal{A}_{n b} \mathbf{U}_{n b}\right)_{f}}+\overline{S_{\phi, f}}
\end{aligned}
$$

where $\mathcal{A}$ is the complete matrix associated with the central coefficients. Re-arranging Eq. A.36, to solve for $\mathbf{U}_{f}$, gives:

$$
\mathbf{U}_{f}=\left(\frac{1}{\mathcal{A}_{f}}\right)\left[\overline{\left(\sum \mathcal{A}_{n b} \mathbf{U}_{n b}\right)_{f}}-(\nabla P)_{f}+\overline{S_{\phi, f}}\right]
$$

Equation A.37 needs to satisfy the mass conservation equation (Eq. A.35). Taking the divergence of the right hand terms of Eq. A.37 and setting this equal to zero (i.e. substituting Eq. A.37 into the mass conservation equation), gives:

$$
\operatorname{div}\left[\frac{(\nabla P)_{f}}{\mathcal{A}_{f}}\right]=\operatorname{div}\left[\frac{\overline{\left(\sum \mathcal{A}_{n b} \mathbf{U}_{n b}\right)_{f}}+\overline{S_{\phi, f}}}{\mathcal{A}_{f}}\right]
$$

Equation A.38 is known as the pressure correction equation and the solution of $P$ from this equation yields the corrected pressure field. Equation A.38 can be simplified by introducing the $\mathcal{H}(\mathbf{U})_{f}$ term:

$$
\mathcal{H}(\mathbf{U})_{f}=\overline{\left(\sum \mathcal{A}_{n b} \mathbf{U}_{n b}\right)_{f}}+\overline{S_{\phi, f}}
$$


The $\mathcal{H}(\mathbf{U})$ term consists of two parts: the "transport part", including the matrix coefficients for all neighbours multiplied by corresponding velocities and the "source part" including the source part of the transient term and all other source terms apart from the pressure gradient. This simplified form of Eq. A.38 is:

$$
\frac{\operatorname{div}(\nabla P)_{f}}{\mathcal{A}_{f}}=\operatorname{div}\left[\frac{\mathcal{H}(\mathbf{U})_{f}}{\mathcal{A}_{f}}\right]
$$

For further information the reader is invited to go through Section 3.8 of Jasak [43], Appendix A of Kärrholm [49] and Chapter 2 of Croft [A1]. 


\section{Appendix A: References}

[A1] T.N. Croft. Unstructured Mesh - Finite Volume Algorithms for Swirling, Turbulent Reacting Flows. PhD thesis, Centre for Numerical Modelling and Process Analysis, School of Computing and Mathematical Sciences the University of Greenwich London, 1998.

[A2] F.H. Harlow and J.E. Welch. Numerical calculation of time-dependent viscous incompressible flow of fluid with free surface. The Physics of Fluids, 8(12):2182 2189, 1965. 


\section{Appendix B}

\section{OpenFoam code}

\section{B.1 "boussinesqBuoyantFoam" Solver}

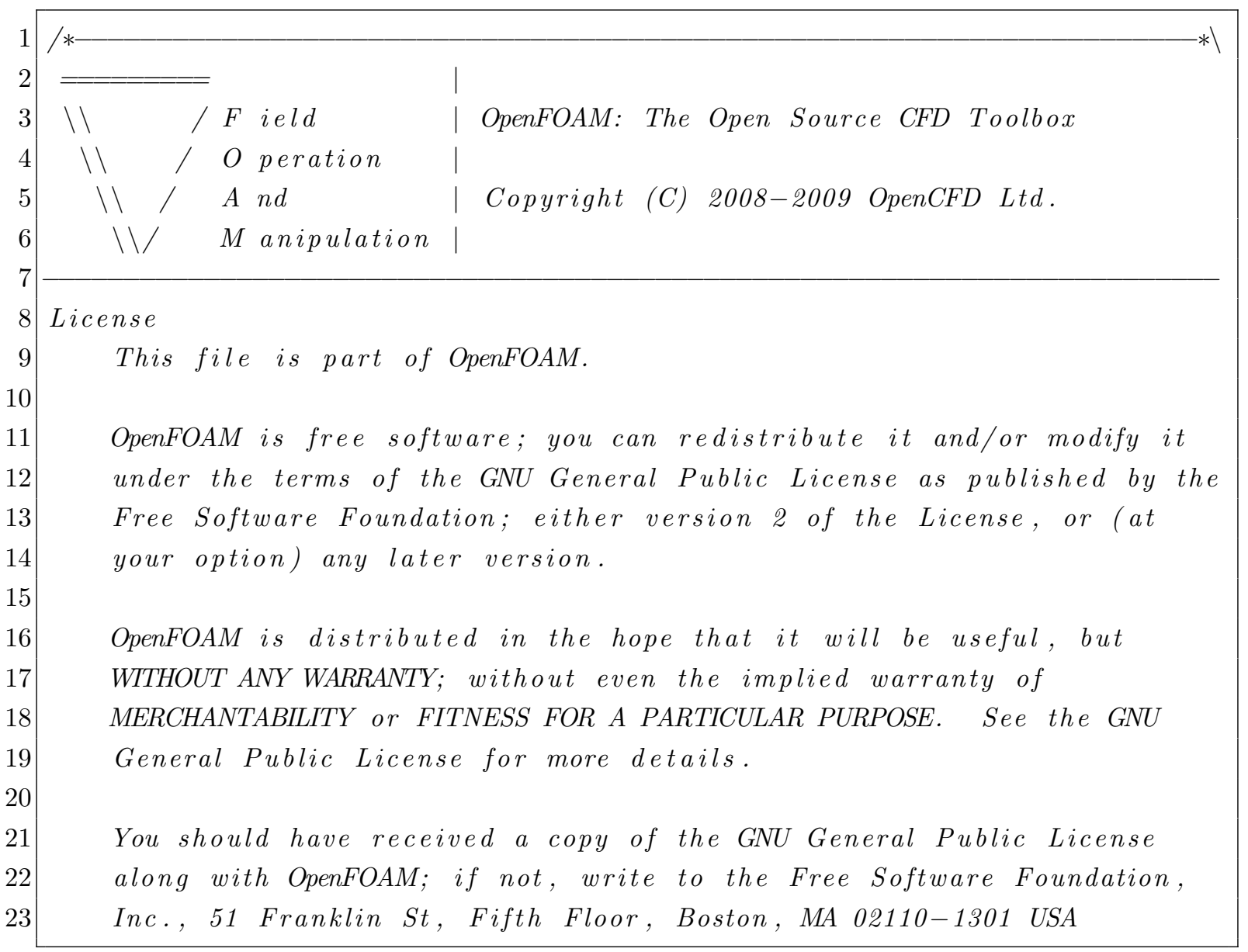




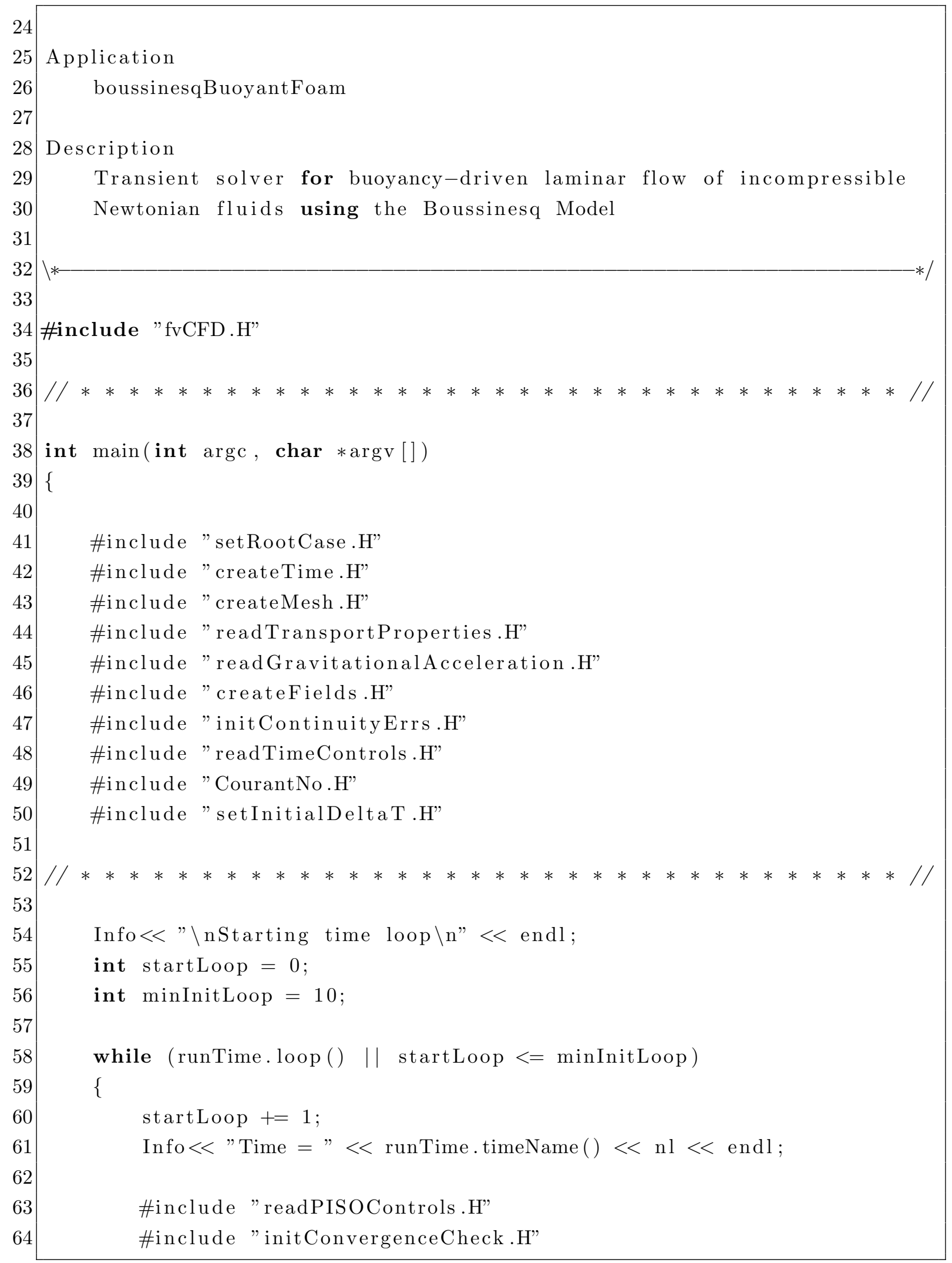




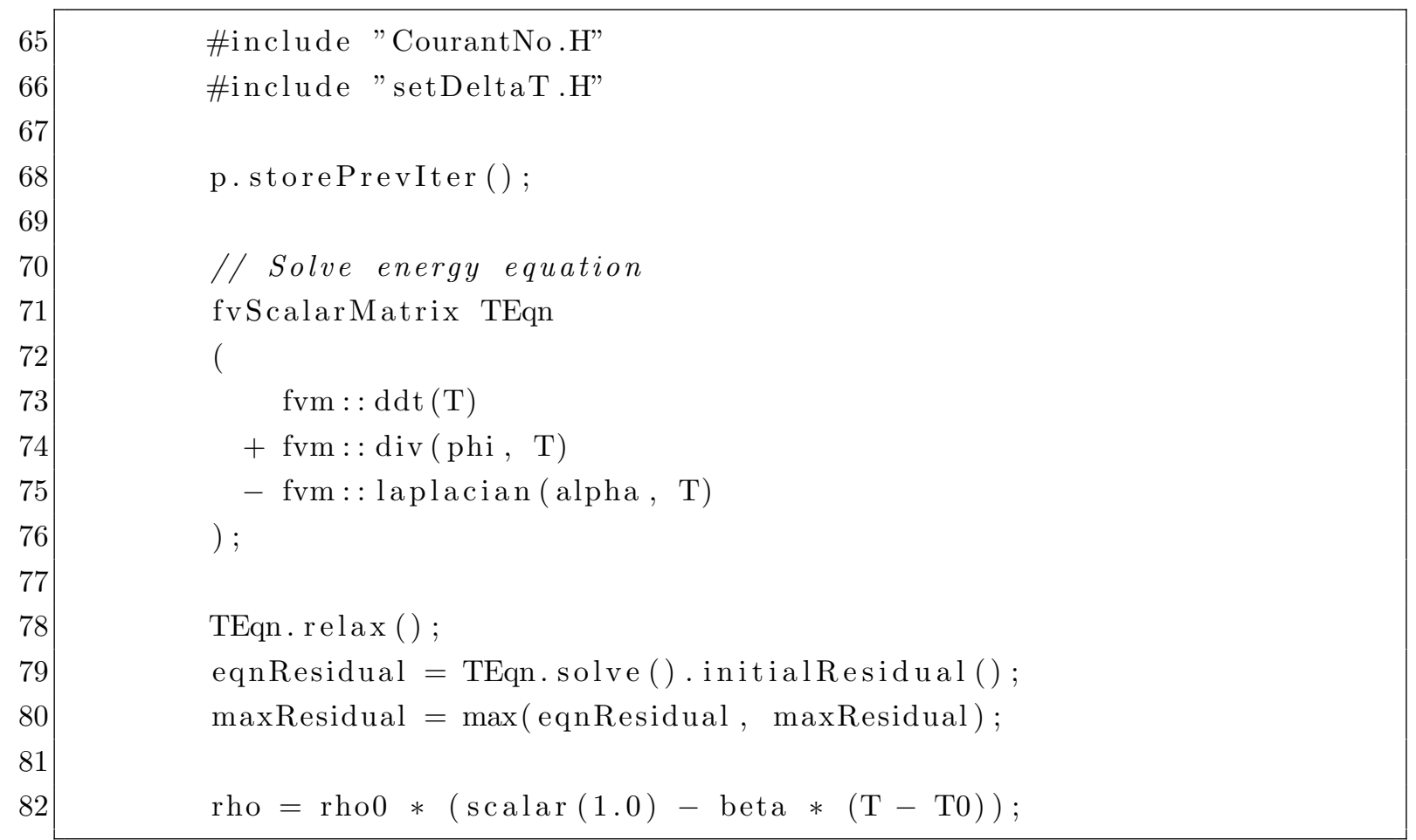

Lines 71 to 76 shows how the energy equation (Eq. A.3) is implemented in OpenFoam. The "fvm::" statements tells OpenFoam that the term following the statement must be determined implicitly. Lines 82 shows the calculation of the fluid density approximation (Eq. 3.7).

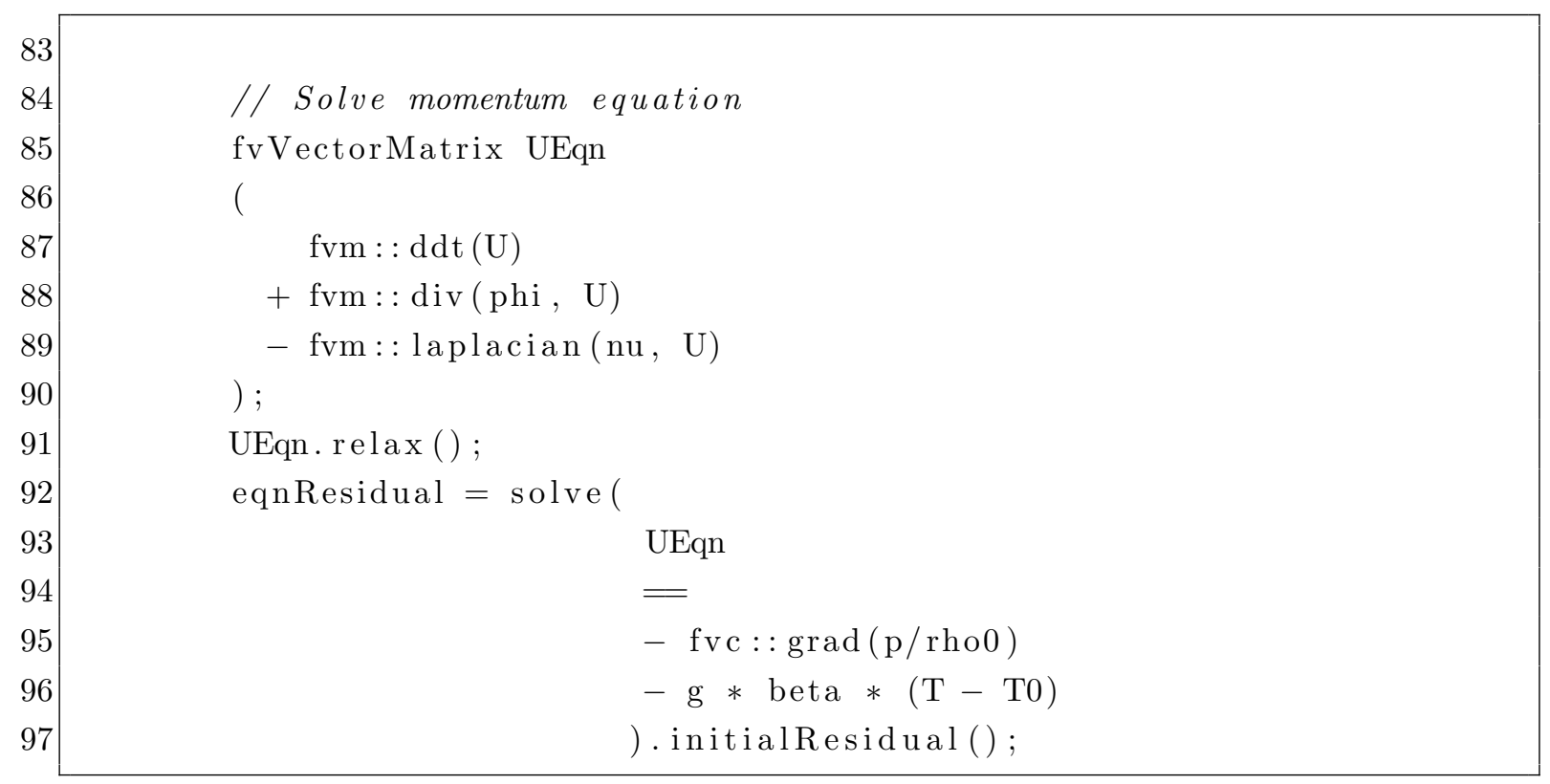




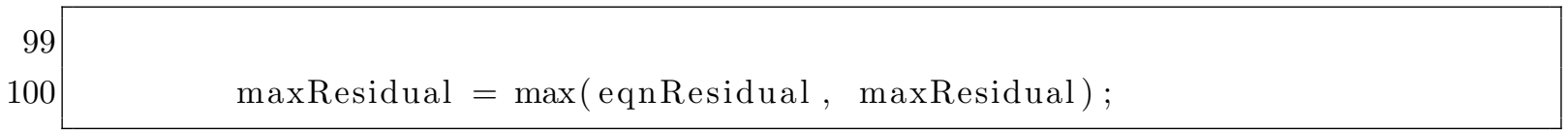

Lines 85 to 90 shows how the momentum equation (Eq. 3.8) is implemented in OpenFoam. The pressure $\left[(\nabla P) / \rho_{0}\right]$ and buoyancy force terms $\left[\mathbf{g} \beta\left(T-T_{\infty}\right)\right]$ are added to the right hand side of the momentum equation and solved in lines 93 to 98. Here the "fvc::" statements tells OpenFoam that the term following the statement must be determined explicitly.

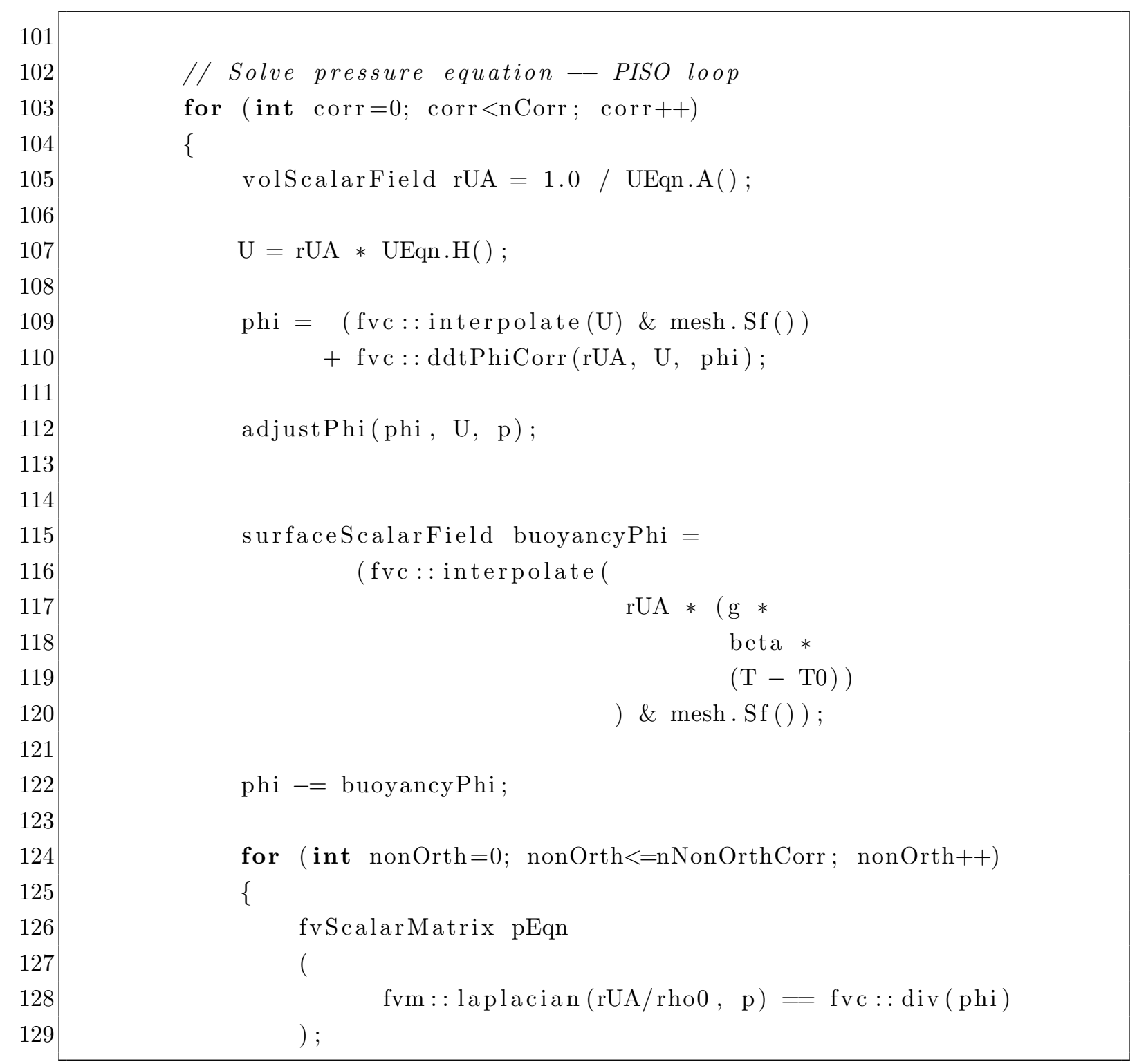




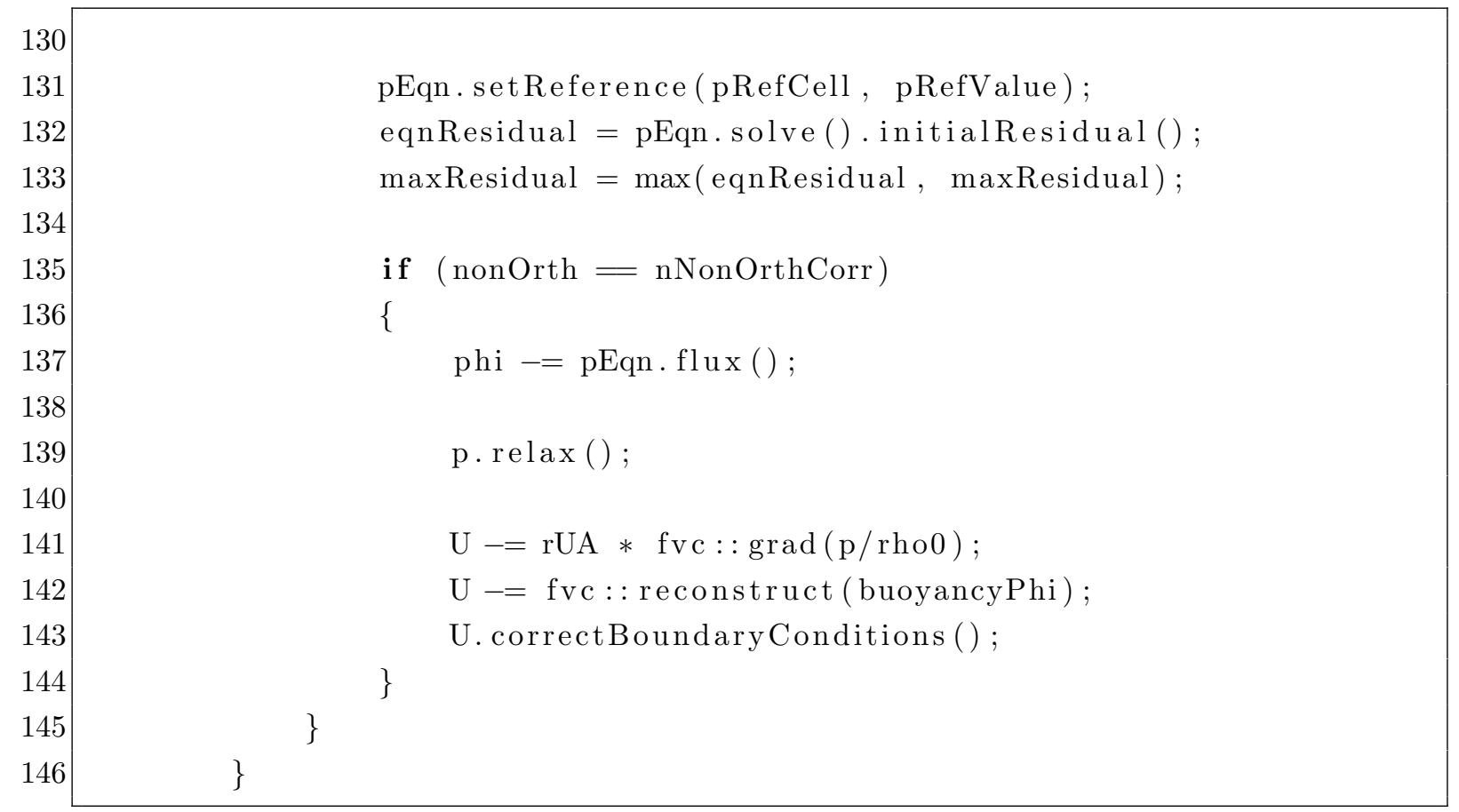

Lines 103 through 146 shows how the PISO algorithm (Section 3.5.2) is implemented in OpenFoam. Lines 105 to 107 represents the first term on the right hand side of the following equation, (cf. Eq A.37):

$$
\mathbf{U}_{P}=\left(\frac{1}{\mathcal{A}_{P}}\right)\left[\mathcal{H}(\mathbf{U})_{P}-(\nabla P)_{P}\right]
$$

which is then interpolated at the cell face centres (Line 109) to obtain $\mathbf{U}_{f}$.

From the definition of $\mathcal{H}(\mathbf{U})$ (cf. Eq. A.39), it can seen that this term should include the source part of the buoyancy force term. However when looking closely at the definition of the momentum equation, implemented in OpenFoam (Lines 85 through 90), it is seen that the source part of the buoyancy force term is absent. This is corrected by first calculating the buoyancy force at the cell face nodes (Lines 115 to 120) and then subtracting this from $\mathbf{U}_{f}$ (Line 122).

Lines 126 to 129 shows the implementation of the pressure correction equation (Eq. A.40) in OpenFoam. The solution of which gives the corrected pressure field. The velocity at the cell face nodes is then corrected, based on the corrected pressure field (Line 137). Finally the centre node velocity is corrected, based on the corrected pressure field 
(Line 141) and based on the absence of the buoyancy force term source part (Line 142).

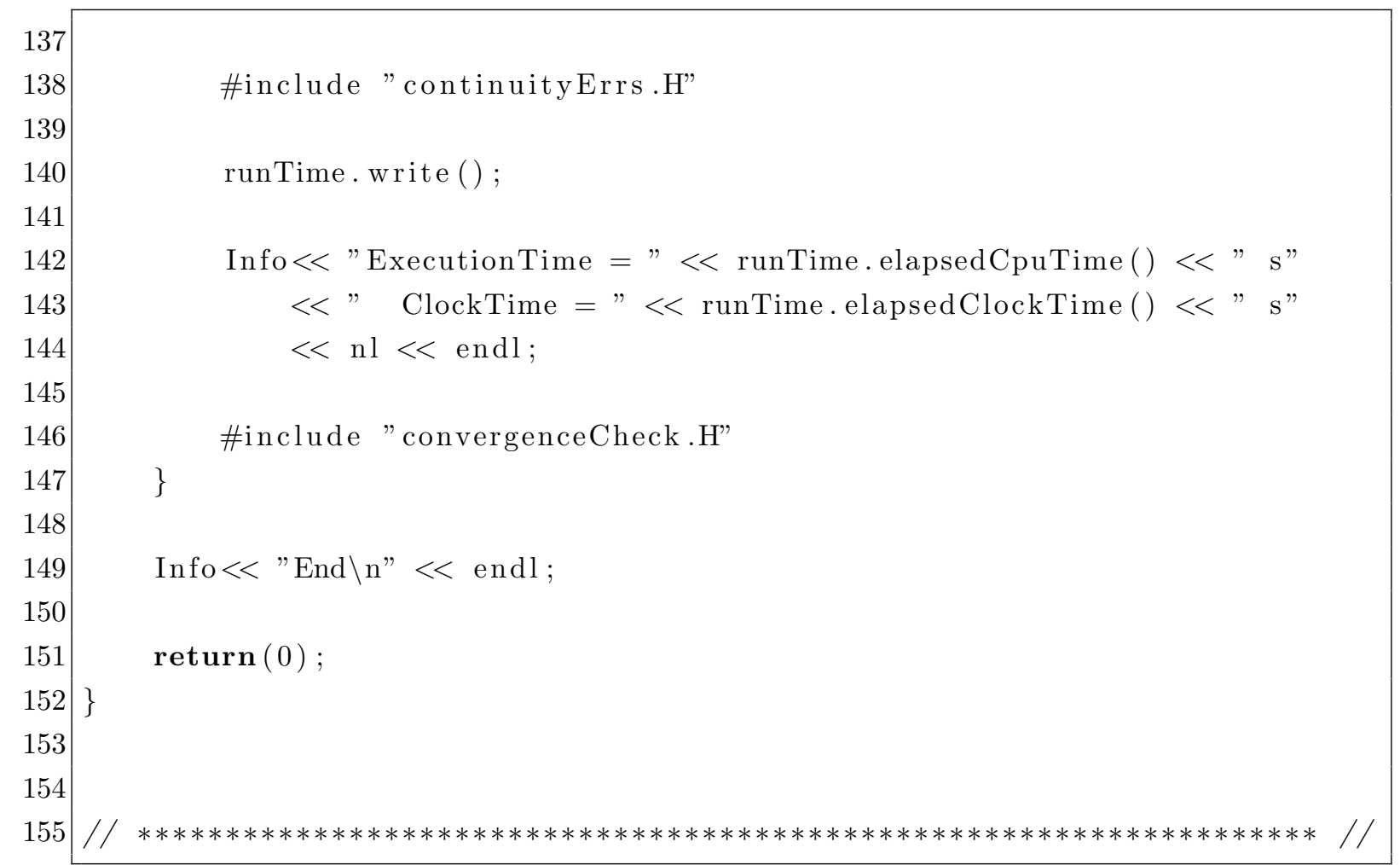




\section{Appendix C}

\section{Data attachment}

\section{C.1 Overview}

Numerous files, images, animations, python scripts and documentation have been attached digitally on DVD at the back of this thesis. Section C.2 summaries the directories attached on the DVD.

\section{C.2 Summary of directory structure}

\begin{tabular}{|l|l|}
\hline Directory & Description \\
\hline $\begin{array}{l}\text { DVD Drive } \\
\qquad \text { Case Files }\end{array}$ & $\begin{array}{l}\text { Case files for several flow regimes and cylinder rotation } \\
\text { speeds for the OpenFoam simulations. }\end{array}$ \\
$I_{\rightarrow} \triangleright$ Documentation & $\begin{array}{l}\text { Documentation, generated using Epidoc [C1], describing } \\
\text { each class and function for the Python Code. }\end{array}$ \\
\hline
\end{tabular}




\begin{tabular}{|c|c|}
\hline Directory & Description \\
\hline \multicolumn{2}{|l|}{ DVD Drive (continued) } \\
\hline \multicolumn{2}{|l|}{$I_{\rightarrow}$ Gallery } \\
\hline$\stackrel{\perp}{\triangleright \text { Animations }}$ & $\begin{array}{l}\text { Temperature and velocity animations for } \\
\text { several flow regimes and cylinder rotation } \\
\text { speeds. }\end{array}$ \\
\hline$I_{\rightarrow}$ Contour Plots & $\begin{array}{l}\text { Temperature and velocity contour plots } \\
\text { for several flow regimes and cylinder ro- } \\
\text { tation speeds. }\end{array}$ \\
\hline \multicolumn{2}{|l|}{$I_{\rightarrow}$ OpenFoam Applications } \\
\hline \multicolumn{2}{|l|}{$\mid \rightarrow$ Solver } \\
\hline$I_{-} \triangleright$ boussinesqBuoyantFoam & $\begin{array}{l}\text { OpenFoam solver "boussinesqBuoyant- } \\
\text { Foam", presented in Appendix B. }\end{array}$ \\
\hline \multicolumn{2}{|l|}{$\stackrel{I}{\longrightarrow \text { Utilities }}$} \\
\hline$I_{\rightarrow} \triangleright$ wallHeatFluxLaminar & $\begin{array}{l}\text { OpenFoam utility "wallHeatFluxLami- } \\
\text { nar", for calculating the heat transfer rate } \\
\text { for each cylinder. }\end{array}$ \\
\hline$I_{\rightarrow}$ Python Code & $\begin{array}{l}\text { Python scripts for interfacing with Open- } \\
\text { Foam for parametrisation and optimisa- } \\
\text { tion, as shown in Fig. } 4.1 \text { and Fig. } 4.4 \text {. }\end{array}$ \\
\hline
\end{tabular}




\section{Appendix C: References}

[C1] E. Loper. Epydoc - automatic api documentation generation for python, 2006. URL http://epydoc.sourceforge. net/. [Online; accessed 04-Jan-2012]. 